\title{
GERI SPAROVEK
}

Engentieiro Agronomo

Orientador: Frof. Dr. Andre Martin Louis Neptune

Dissertaço apressentade a Escola Superior de Agricultura "Luiz de Quedroz" "da Universidade de sao Paulo, para obtencaro do titulo de Mestre em Agronomia, Area de Concentracaso: Solos e Nutricto de Plantas

\author{
$P I F A C I C A E A$ \\ Estado de Saro Paulo -... Erasil
}

Maio - 1998 


\section{Sparovek, Gerd}

S736a Avaliação agronômica do suporte sólido capí lar para fertilizantes fluidos. Piracicaba, 1988.

$117 \mathrm{p}$.

Diss.(Mestre) - ESALQ

Bibliografia.

1. Adubo líquido - Avaliação I. Escola Supe rior de Agricultura Luiz de Queiroz, Piracicaba

CDD 631.8 
AVALTACÃO AGRONOUMICA. DO SUPORTE SÓLIDO CAPILAR PARA FERTILIZANTES FLUIDDOS

GERD SPAROVEK

APROVADA EM: $\quad 11 / 07 / 88$

COMISSÃO JULGADORA:

PROF. DR. ANDRE MARIIN LOUIS NEPTUNE - ESALO/USP

PROF. DR. RONALDO IVAN SILVEIRA - ESALQ/USP

PROF. DR. EDSON LUIS MENDES OOUTINHO - FCAVJ/UNESP

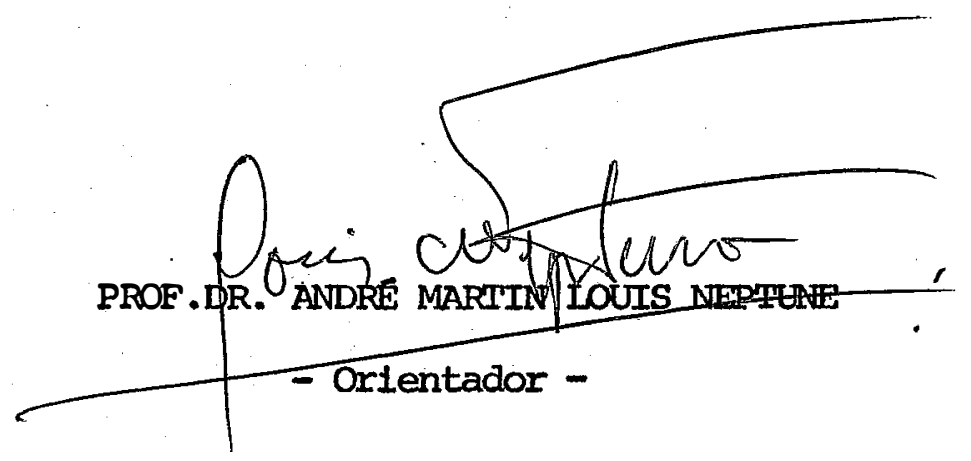




\section{AGFADEC IMENTOS}

Ao Prof. Dr. Andre Martin Louis Neptune pela

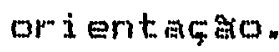

A ULTFAFERTIL pela cessa dos Suportes Solidos Capilares para Fertilizantes Fluddos e suporte financ:

A FAFESF pelo gupomte financeimo.

Ao Eng. Agr "Luciano Fin. Cintra pelo ausidio nit parte esperimention.

Aos colegis Eurdides Malheipos Jost Carlos Earbosa da FCAVJ -UMESP pela orientaça nas andises Estatisticis

Aos funciomarios do Depto. de Solos, Geologia - Fertilizantes da EEALQmugF do Depton de Solos e Adubos da FCAVJ-UNESF que ausili aram na mealizaça do trabal ho.

A todom outros qus de alguma forma contribuiram,

MELS sincerom agraderimentos. 


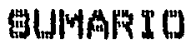

Página

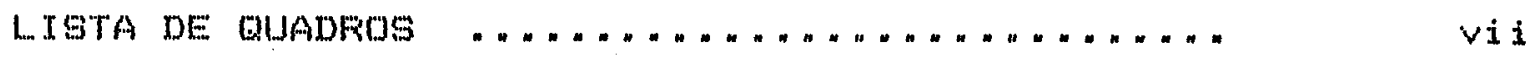

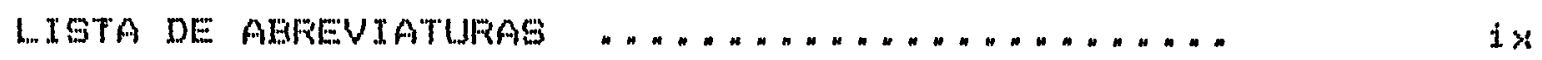

RESUMU

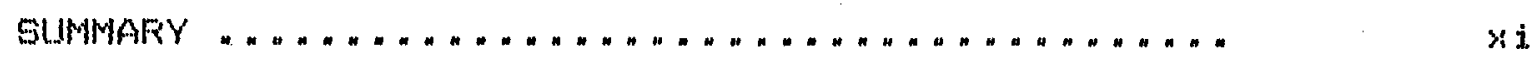

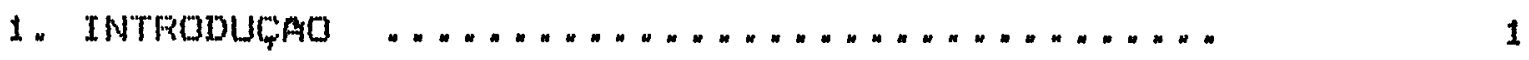

2. REVTSAO DE LITEFATURA ....................

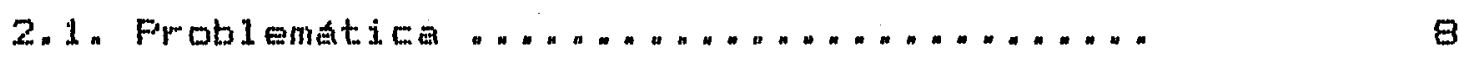

2.2. Tipos de fertilizantedesolubiliza-

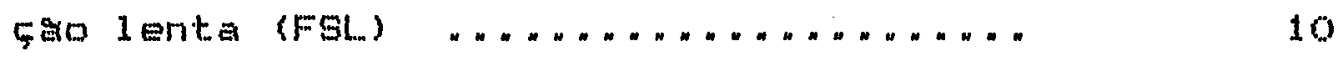

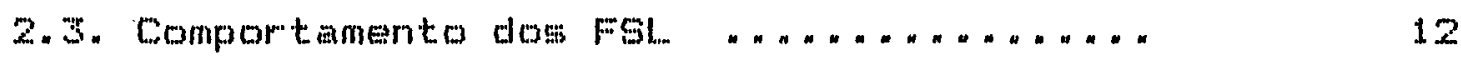

2.4. Manejo dos FSL . . . ........................ 16

2.

2.5.1. Resultados favoraveis ......... 19

2.5.2. Resultados desfavol dvei ......... 21

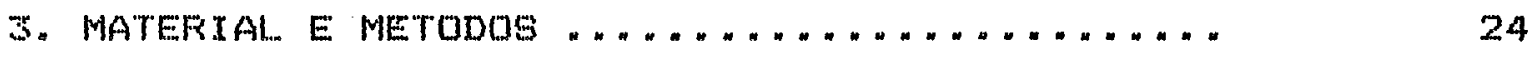

3.1. Suporte capilar pora fertilizantess

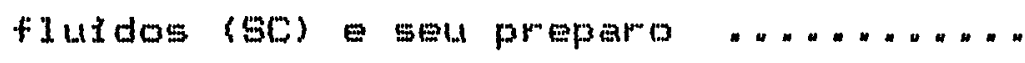

3.1.1. Freparo do $8 \mathrm{C}$ noe ensaios de efeito residual e de micronutrienteg $\ldots \ldots \ldots \ldots \ldots \ldots \ldots \ldots \ldots \ldots \ldots$

5.1.2. Prepra do 50 no mensio de $1 \mathrm{j} \times \mathrm{i} \vee \mathrm{i}$ acs 
Página

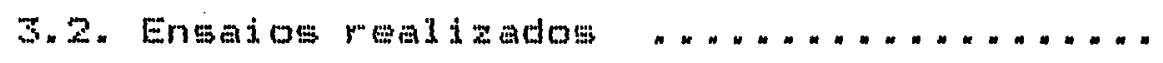
28

3.2.1. Erisaio de efeito residual. "....

30

3.2.1.1. Tratamentos básicos "..

$\$ 0$

$3.2 .1 .2, D 0 E a s d e N, F \in K \ldots \ldots$

32

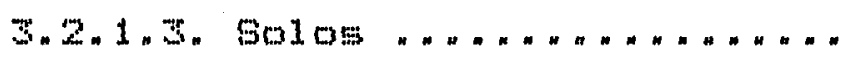

32

3.2.1.4. Calagem ..............

34

3.2.1.

sis

3.2.1.6. Cortes .............

3.2.1.7. Tratos e consideraCBess gerais .........

3.2.1.6. Delineamento experiment. 1

3.2.2. Engaio com micronutrientes "...

3.2.2.1. Tritamentos basicos "..

3.2.2.2. Doses . ...............

$3.2 .3 .35010 \ldots \ldots$

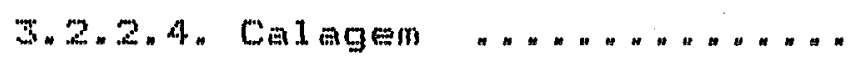

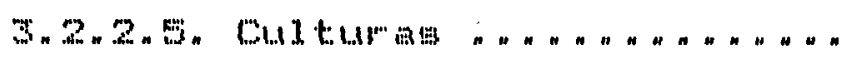

3.2.2.6. Molmeitio

3.2.2.7. Tratom e tongider a Goes gerais

3.2.2.6. De1inemento experimen$t=1$ 
Pagina

3.2.3. Ensaio de 1ixiviaca "..........

3.2.3.1. Tratamentos butcos "..

3.2.3.2. Insta1 acko do Ensaio ... 46

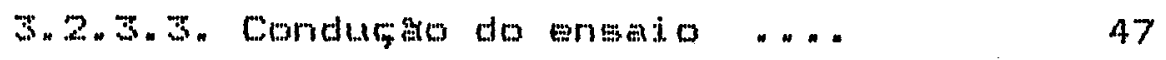

3.2.3.4. Colleta das amostras ... 48

3.3. Metodologia analitica ............... 48

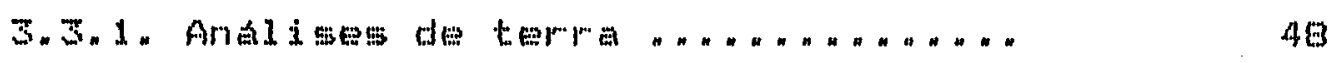

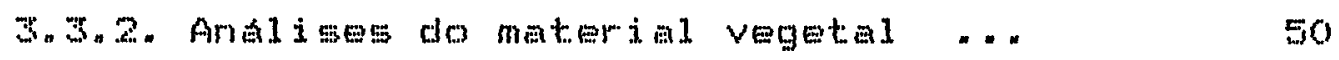

4. FESULTADDS, DISCUSSAO E CONCLUSGES

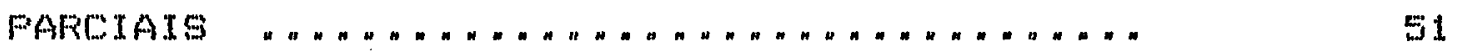

4.1. Ensaio de efeito mesidual "..........." 51

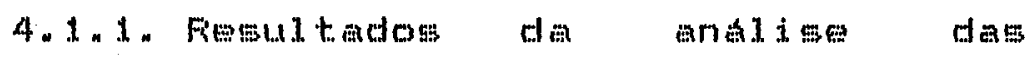

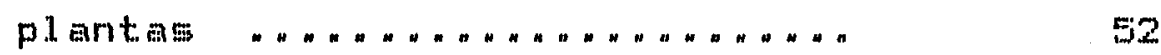

4.1.1.1. Materia

$M \ldots S_{\ldots} \ldots \ldots \ldots \ldots \ldots \ldots$

52

4.1.1.2. Nitmogenio (\% na folha)

N $\ldots \ldots \ldots \ldots \ldots \ldots \ldots \ldots \ldots$

55

4.1.1.3. Fosforo (\% na folha)

P ....................

54

4.1.1.4. Fotassio (\% na foll o

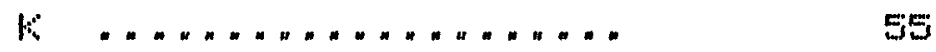

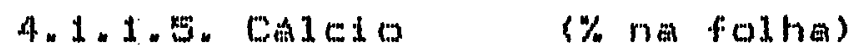

C.

56 
4.1.1.6. Magnesio (\% na folha)

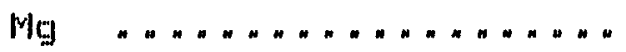

4.1.1.7. Manganes (ppon na foltha)

$M n \ldots \ldots \ldots \ldots \ldots \ldots$

58

4.1.1.9. zinco (ppon ne folha)

Zn

58

4.1.1.9. Ferro (ppm na foltha)

$F \in \ldots \ldots \ldots \ldots \ldots \ldots \ldots \ldots$

4.1.2. Resultados das amalises de term $\ldots \ldots \ldots \ldots \ldots \ldots \ldots \ldots \ldots \ldots \ldots \ldots$

4.1.2.1. Atividade do hidrogernio

$$
\text { (pH CaCl) - } \mathrm{CH} \ldots \ldots . . . .
$$

4.1.2.2. Fomforo Boldvel (mg/cm)

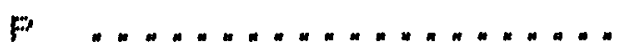

4.1.2. 3. Fotassio

trocavel

$$
\left(m e q / 100 \mathrm{~cm}^{3}\right)-k
$$

4.1 .2 .4$.

$$
\begin{aligned}
& \text { Walcio trockvel } \\
& \text { (meq/100 } \mathrm{km}, \text { - C. ".." }
\end{aligned}
$$

4.1.2.

trocinel

$$
(m \in q / 100 \mathrm{~cm}), \mathrm{Mg} \ldots . .
$$

4.1.2.6. Soma de Basea

$$
\begin{aligned}
& \text { I } \\
& (m \in q / 100 \mathrm{im}) \ldots 5 \ldots
\end{aligned}
$$


Pagina

4.1.2.7. Capacidade de troca de (witions $\quad(m e n / 100 \mathrm{~cm})$

Citc

4.1.2.8. Satur mo por bases (\%) $V \%$

4.1.2.9. Enxofre

$150^{-2}$ 4g/100 cin'

50

4.1. 64

4.1.4. ConelusBes do ensedio ........... 69

4.2. Ensmio com micronutmientes " " . " " . "... 79

4.2.1. Apresentacia dos pesultados .... 79

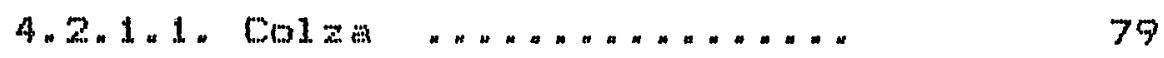

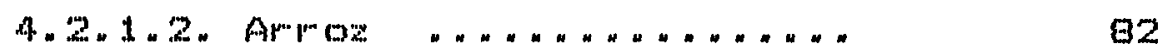

4.2.2. Di.muscuso doe resultados ......

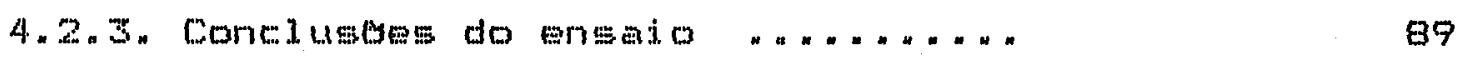

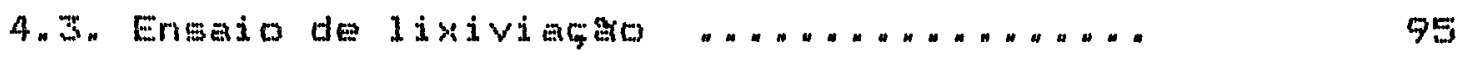

4.3.1. Apresentacio dos resultados ....

4. 3.2. Di

4.

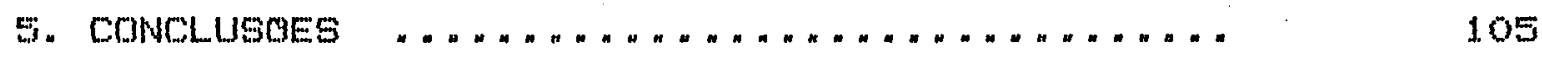

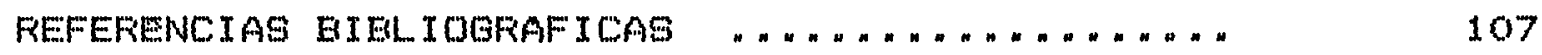




\section{LISTA DE DUADFDS}

Página

1 -.. Madias geriais dos tratamentos no ensaio de

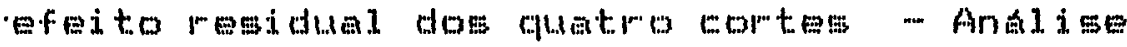

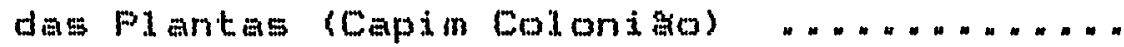

2 - Inter aço entre os to atamentos do ensaic de efeito residuml - Analiste das Flantas dos quatro cortes (Capim Colonigo) " " "........

s-Interacoss entre as fpocas de corte e os tretamentos no ensaio de efeito residual - Analise das Flantag (Capion Colonita) .....

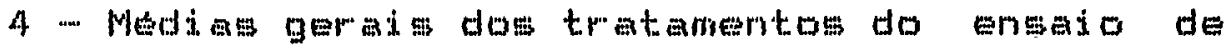
efeito mesidual … Andi

5 - Interaço entre tratamentos do ensaio de efoito residul m Andlise das termas ......

6 - Interara entre tratamentos no monjo de mieronutrientes. Analise da parte abrea da $\operatorname{col} 2 a$

7 Interaça entre tratamentos no ensaio de micronutrientes. Anelise da parte asper do $\operatorname{arm} 0 z$ 
Pagina

8-Resultados da andise de terma do ensaio de

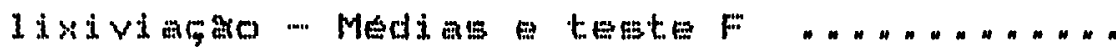
102

9 -.- Regultados da analise de terra do ensaio de lixivi argo - Resultados rom interm ficativa entre Tratamentos e

Profundidade . . . . . . . . . . . . . . . . . . . 


\section{LISTA DE AEREVIATURAS}

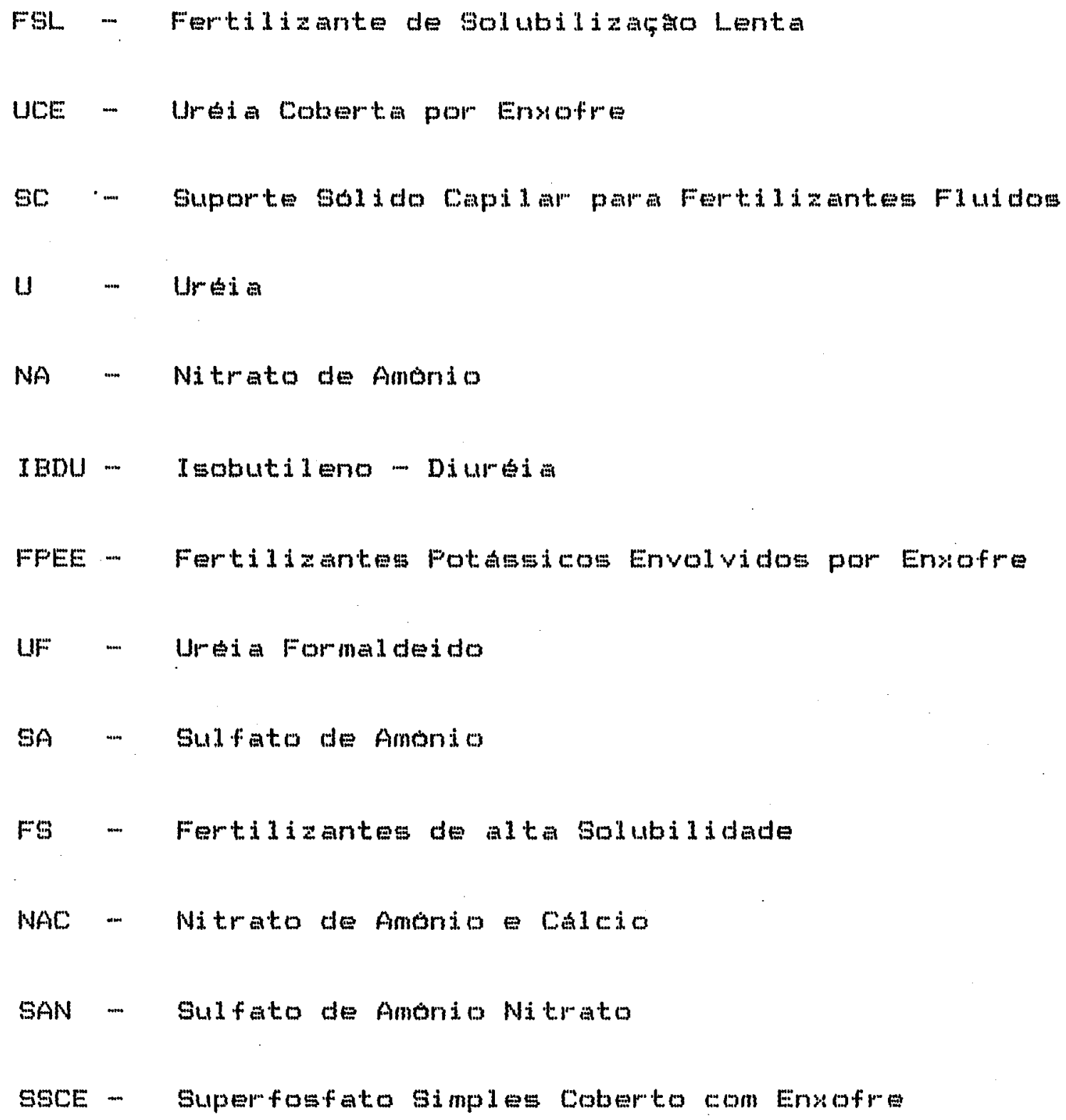




\section{AVALIAGAO ADFONOMICA DO EUPOFTE BOLIDO CAPILAR PARA FERTILI ZANTES FILUIDDOS}

AltoI GEFD SFARCIVEK:

Orientador: PFOF. DF. ANDKE MARTIN LOUIS NEFTUNE

RESUNO

Proturoume avaliar o comportamento agmonomico do Suporte Solido Capilar para Fertilizantes Fluidos (GC) - efeito sobre as plantas e sobre os 50105 alem

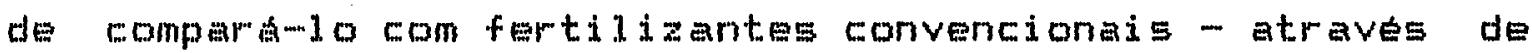

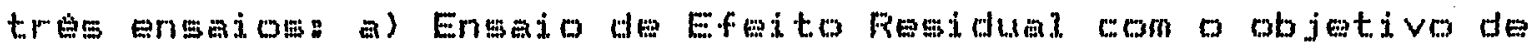
Verificar seu comportamento de Fertilizante de Solubilizasa Lenta (FGL): b) Ensaio com Mi cronutrientes com o objetivo de avaliar a possibilidade de aplicaco de micronutrientes via Sc $e$ E) Ensaio de Lixiviacar, fin de verificar a Iisiviaço de Extions devido à presenca do sc no solo.

0 SC nå apresentou caracter Iticas de FSL, mostrou-se viavel na aplicaço de micronutrientes no 5010 e nao provocou a 1 ixiviaço de cations ou a neutralizarao de Al ${ }^{+3}$ em profundidede.

*

o 50 e Lm produto fertilizarite experimerital desenvolvido PEI AL ULRAFERTIL. 
AGRONOMIC EVALUATION OF THE SOLID CAPILAR EUPFORT FOR FLUID FERTILI IZERE

Author: GERD SFAROVEK Adviser: FROF. DR. ANDRE MARTIN LOUTS NEFTUNE

SUMMAFY

Ey conducting tree experiments the agronomic behaviour of the Golid Capil ar Support for Fluid Fertilizers * (SC) was evaluated: a) Residual Effect Experiment with the objective to verify the behaviour as 91 ow Release Fertilizer (SRF): b) Micmomutrient experiment with the objective to evaluate the poseibility of application of mieronutrients via SC and, E) Leaching Experiment with the objective to verify the leaching of Eation due the presence of the SC in the goil.

The SC did not demonetrate FGL caracteristics it is a vimble way of applying micronutmients to the soil, and did not induce leaching of cations or the neutralization of desep placed Al *

SC is an experimental fertilizer developped by UL.MTRAFERTIL. 


\section{INTRODUCAO}

Defineme a regja tropical como sendo. a parte do globo onde a variaço da temperatura media mensal dos tres meges majs quentes do ano difere em menow de so da temper atura media dos tres meses mais frios. Esta regiơ estendem geograficamente do equador a 23,5 Nomte ou Sul, aprosimadamente.

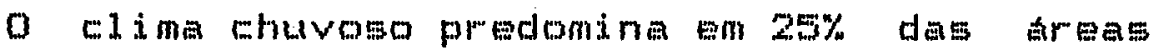
tropicais e o clima estacional, com uma estacao seca

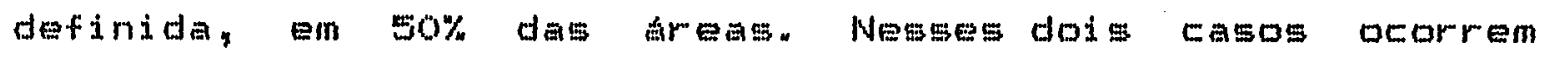
Ehuvas de alta intensidacle curta dur aço.

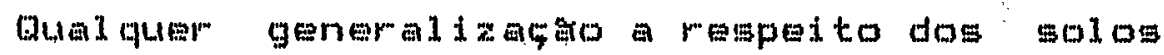
dessa regio e no minimo perigosa. Afirmar que todos os

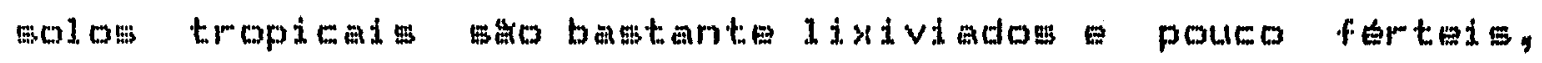
seria, o mesmo que dizer que todos os solos temperados săo jovens e ferteis (SANCHEZ, 1981). Apesar disso, sen dúvida alguma, uma parte consideravel dos solos tropicais reune as seguintes caracteristicas: a) avangado estagio de 
intemperismo, consequentemente baisia meserva minerala b) baixa caparidade de trore de cations devido et dominancia de argila de atividade baisae baiso teor de materia organica: c) alta intensidade de lixiviacaro, devido a boa permeabilidade e a elevada intersidade das chuvas e d)

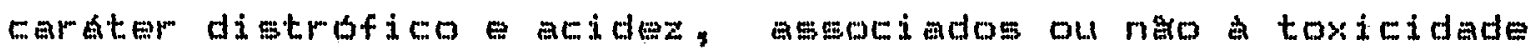
de Aluminio.

Em resumo, sato solos que necessitam de comregBes, principalmente do ponto de vista quimico, para se tornarein mais produtivos.

Dessar forman, nos tropicos os rendimentos dos cultivos $\mathrm{e}$ a producto de alimentos podem aumentar drasticamente mediante aplicace do de tenicas modernas.

Felo exposto acima, fica 01 ama a necessidade de cormigir o solo e molubar wo plantas pirm atingir niveis razouveis de produço en grande parte das terras dos $\operatorname{trmspicos}$

Oos fertilizantes nommalmente utilizados na regia tropion tem en comum com aqueles de regibes temperadas sua alta solubilidade em agua. Na majoria dos 
fertilizantes, os elementos fertilizante estao presentes na

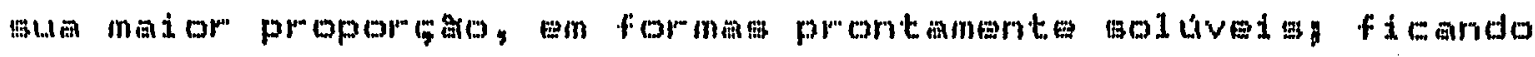
assim imedi atanente disponiveis a absorcto pelas raizes das plantas. Entretanto temos o meguinte a considerar: a) as plantas no absorvem os rutrientes de uma vez, fazem-no ao longo de todo o seu cicion b) estando dissolvidos, os nutrientes nå ser das plantas, participara dos processos quimicos, fisicoquimicas e biologicos do solo como um todo e c) esses processos levam, principalmente no ambiente tropical, a perdas ou a uma indisponibilizaço dos nutrientes em relaça As plantas cultivadas.

Em relagro ao nitrogeniog metade do $N$ aplicado pode ser perdido por lisiviacton volatilizacăou imobilizacto, sendo lisiviago o processo mais importante (ALLISON, 1966). Como vimot, a ixiviacio assume intensidades muito promunci ades nos tropicos.

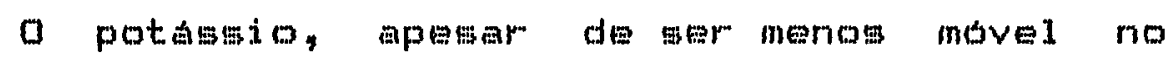
solo, tambdom pode sofrer perdat por liniviacao. A fisacaro ou imobilizacto do ki nas condiçbes troptiais nao assume papel i mportinnte. 
Nomalmentu, para evitar essas perdas, prineipalmente a lisiviarso do nitrogenio, procede-so o parcelmmento da aplicasto, ao longo do ciclo da cultura.

0 fosforo, apesar de nă ser lixiviado em condicibes normais, 11 gamse muito fortemente aos oxidos de fermo coristalinos, ficando ligado ou fixado a esses mi nerais de forma priticamente irreversivel.

A maneilia encontimada de contornar esse problema 4 mplicar dowes multo superiores as necessamias ou exigidas pelas plantas diminuip o contato do fertilizante com o solo.

Uma outra maneira de amenizar esses problemas, prineipalmente perda de $N=K$ por $1 i$ aviaço em solog de bai\%a atividade de cargase sujeitos a chuvas muito intensas, seria reduzir a solubilidade dos fertilizantes (ENGELSTAD \& FUSSEL. 1971).

A libermga lonta dos nutrientes, mo longo do

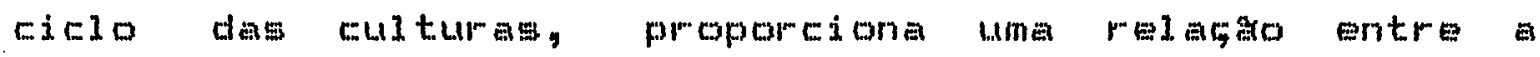
quantidade absorvida pelas plantas e a quantidade perdida

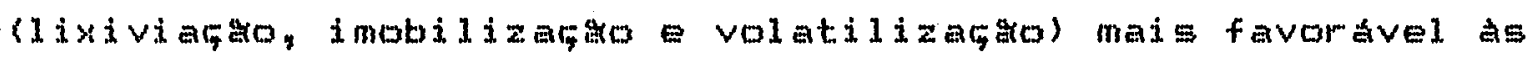


plantas, - aumentando a eficiencia dos fertilizantes. 0

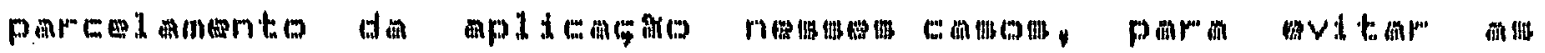

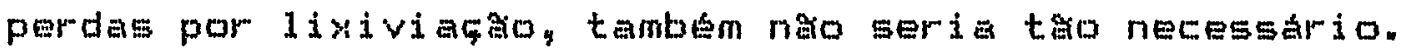

Diversos Fertilizantes de Solubilizaço Lenta (FSL), principalmente nitrogenados, tem sido estudados e utilizados nas mais distintas condicBes.

No Erasil a Utilizaçăo en escala comercial de FGL nitrogenados ou potassicos ainda inesiste poucas pesquisas vem sendo conduzidas. o numero de trabalhos publicados por autores nacionais respeito de FEL e muito pequeno fmente a complesidate potancialidade despen produtos.

Qes produtos tem potentialidade de utilizaça como FSL apmesentam duas limitaç⿻上丨, apontadas na maioria

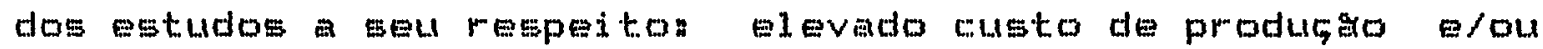
eficácia questionável pouco previsivel.

Nesse contento, a busea de novos produtos fertilizante, com potencialidace de aprementarem um comportamento de Solubilizaca Lenta, de eusto baiso e utilizando materia prima dimponivel com facilidade e de 
extrema importancia.

A WhTRAFERTIL dESErVoIVEU em carater

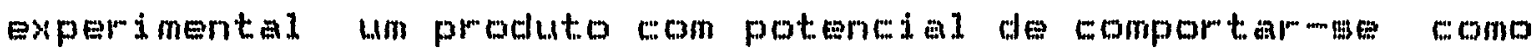
um Fol, Ehamado de Suporte Capilar para Fertilizantes Fluidos ou SC. 0 SC consiste de um wilindro de compatendo, formando um tompo sdido e poroso, que imerso

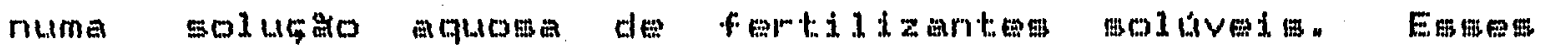
fertilizantes solliveis, absorvidos pelo sC da soluça,

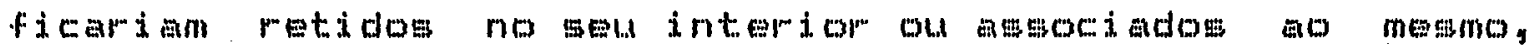

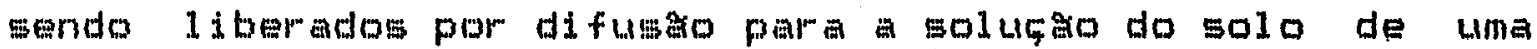
maneima mais lenta.

Partindo da hipotese de que o $S E$ e um fertilizante de solubilizaço lenta em potencial e a fim de avaliam o comportamento dese novo produto fertilizante,

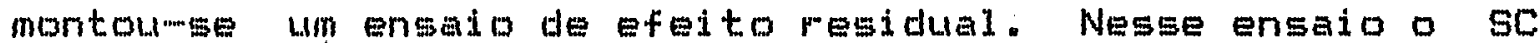
foi comparado a formas sol Liveis.

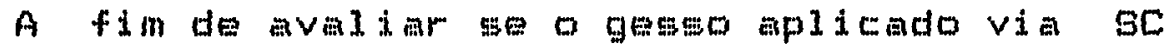
provocamia a Iixiviaço de outmos cations foi conduzido um ensaio de lixiviaro em colunas de solo.

Dada a dificuldacle de aplicaço uniforme de 
mitronutrientes na forma solida no solo a facilidade com

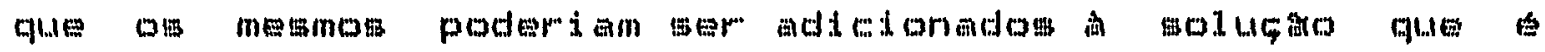
abmorvida pelo se, conduziumbe um ensaio a fim de avaliar a possibilidade de aplitaço de micmonutmienters vi.a SC. 


\section{REVISAD DE LITERATURA}

\subsection{Problematic}

Em extenso trabalno de revisao bibliografica, a respeito do uso de fertilizantes en condicBes tropicais, ENGELSTAD \& RUSSEL (1975), concluen que os maiores problemas nessas condicbes sô a lixivi acto e a fixaço dos nutrientes aplicados via fertilizantes. A lisiviarto do $N$ e do k pode ser resolvida diminuindo-se a solubilidade, consequentemente a Iiberaço desses mutrientes. Cobrir formas solúveis de fosforo com enxofre pode reduair gua fixacion.

Estudando o destino das formas de nitrogenio aplicadas ao solo, ALLISON (1966), afirma que na maioria dos casos apenas a metade do $N$ aplicado e aproveitado pelas plantas. 0 restante perdido por lixiviaga, volatiliz m5a ou imobilizaço, sendo a lisiviagơo a responsavel pela maior parte das perdas. A maneira de solucionar o problema, segundo o autor, fornecer apenas o necessurio ass plantase em doses que possam mer absorvidas prontmente. Decorre dai a necessidade de parcel amento das adubacbes nitrogenadas, 
principalmente quando realizadas com fertilizantes altamente

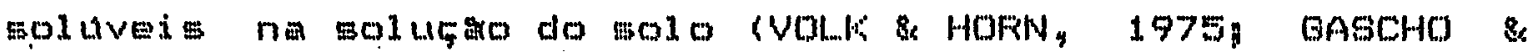
SNYDEF, 1976: SHELTON, 1976: NNADI \& AEED, 1983; FUCHADES Et ali , 1984 E MAQUIEIRA, 1994).

VERSTFAETEN \& LIVENS (1975) consideram quE o fertilizante nitrogenado ideal e aquele que libera o nutriente numa intensidade que se aprosima da necessidade das plantas evitando assim perdas por lixiviacto ou desnitrificacaro. Uma fonte dessas poderia ser aplicada em dose linica premplantio, evitando as coberturas. NNADI \& ABED (1993) afirmam que o uso de formas muto soluveis de $N$ aplicadas en culturas, como o algodá a o sorgo en preplantio, levam a perdas devido a 11भiviacä. Essas fulturas, absorvem muito pouco $N$ no inicio ficando o fertilizante Sujeito a 1 ixivi aço.

A redurgo da solubilidade dos fertilizantes principalmente os nitrogenados, parece ser uma soluço para os problemas acima apontados, minimizando as perdas dos rutrientes e 1iberando-om numa proporcato ligeiramente acima da demanda das $p 1$ antaE CCHTCLINA \& GLORIA, 1990: EGELGTAD \& RUSSEL, 1975 \& TISDALE \& NELSON, 1974).

Em boletim editado pela FAO (1984) indica-se - uso de fertilizantes de solubilizaço lenta para 
culturas de ciclo longo como a Eana-de-açucar e abacaxi e mecomenda-me sempre verificar a rel aço custo/beneficio.

OE golos de baixa Cre e baisa capacidade de tamponamento como e ca caso dos solos das savanas africanas, să quimicamente pouco tamponados, podendo por exemplo ocormer uma acidificaço drastica capaz de diminuir a produço pelo simple uso de mulato da amonio cono fonte de nitrogenio (EACHE: \& HEATHCOTE, 1968 ).

Nesses casos, deve-se estudar nao apenas efeito dos fertilizantes nas culturas, mas tambem avaliar o efeito dos fertilizantes no solo, em ensaios de longa dur a5\% (NNADI \& AEED, 1983).

2. 2. Tipos de fertilizante de solubilizaço lenta (FSL)

De uma maneira geral os fGL podem ser divididos em trme grupos:

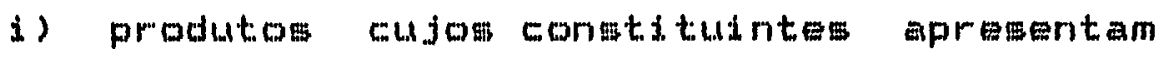
baixa solubilidade na solucoto da solon il) materiais que precisam passar por transformacoes microbiologicas antes de serem disponiveis as plantas e iii) produtos soluveis que foran envolvidos por materiais pouco permeaveis, pouco saluveis ou degradáveis lentamente pelos microorganismos do 
SO10 (JAFFELL \& EDERSMA, 1980).

No primeiro grupo, isto e, fertilizantes que apresentam baixa solubilidade na soluca do solo podemos citar oxamida, isobutileno-diureia, crotonilidina-diureia como fontes de $N$ (PRASAD et alii, 1971). Como fontes de Ko fosfato de amonio potásio k-frita (HDLCOME, 1981) de F fosfato de rocha (ENGELSTAD \& FUSSEL, 1975).

Como produtos que precisam passar por transformaçes microbiologicas antes de serem disponiveis as plantas podemos citar diversas combinacbes de ureia com formaldeido, LImi form (HAAN \& WEFKHOVEN, 1975) $a$ as triazinas (PRASAD et a1ii, 1971).

No litimo grupo, isto f, em produtos golliveis que foram envolvidos por materiais pouco permeaveis, pouco soluveis ou degradaveis lentumente pelos microorganismos do 5olo, podemse utilizar como substucia envolvente polimeros (polietileno, acrlico, acetato e outras mesinas), ceras, parafinas, borrachas, piches, metais evaporados, substancias

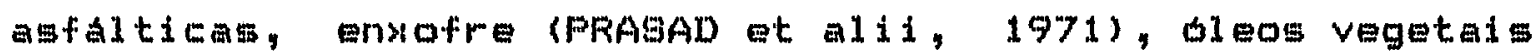
(KHANDELWAZ et alii, 1977) ou acondicionar os produtos em macos plasticos ou de celofane (SNYDEF \& YDUNG, 1974 E PEREGRINE et. a111, 1931). 
A Vermiculita, expandida ou nă, quando tratada com gat de amomia mesulta num produto no qual o $N$ nitrifica de forma lenta. O produto e considerado um escelente meio de cultura tanto pelas proprieddes fisicas como pela lentia liberacaro de N (9TONE : WILD, 1977).

Interessarte trabalho vem sendo feito por HOFOWITZ et ali (1979) no qual os pesquisadores fundem fosforita, micasisto biotita materias primas de ocorrencia comum no nordeste brasileiro - - ven obtendo produtos cujoe testes de solubilidade indicam um comportamento de solubilizaça lenta.

\section{3. Comportammonto dom FL}

Estudionto a liber acto de rutrientes de Fil.

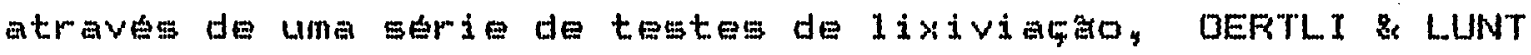
(1962) destreveram o fenomeno como sendo uma difusao na qua ha a entrada de dgua atraves da pelicula que envolve o fertilizante, formando uma soluca saturada que se difunde

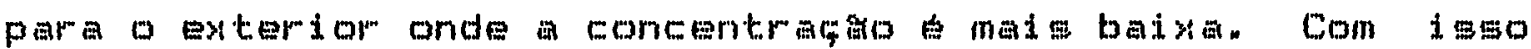

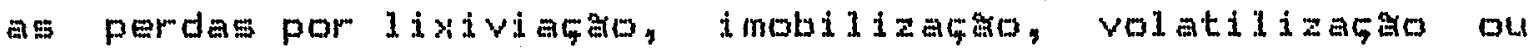

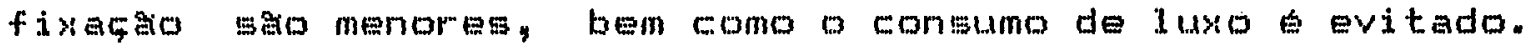
como todo processo de difusa a liberaço de nutrientes pelos FSL Hependente de temperatura. 


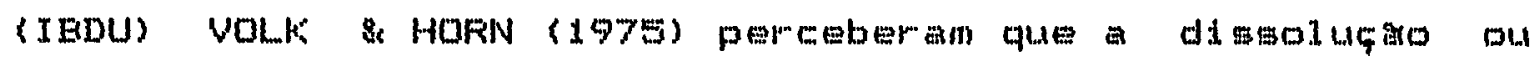
liberaço de nitrogenio foi maior quando a granuląă do

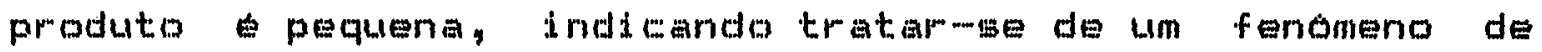
superficie sendo consequentemente regulado pela superficie EEpécifica. FRAgAD et al1i (1971) afiman que para a maioria dos fertilizantes de solubilizata lenta nitrogenados, a liberaço e afetada pel a muperficie exposta.

HOLCOME (1991) Eetudou a liberacaro de potásio de fertiliantem potassicos soluveis envolvidos por ensofre (SCK) E por resina (OSMOCOTE) e observou que a liberacko se da de forma linear" Frodutos potassicos de baixa solubilidade com k-fritas, liberaran muito potamsio inikialmente, desintensifitando-se a $\quad 1$ iberaçัด posteriormente. 0 autor lembra que a absorfơ de potásio pelas plantas praticamente linear. Num estudo semelhante SNYDEF \& GASCHO (1976) trabalharam com Lreia e cloreto de potiasio envolvidos por enxofre rsCU e sck, respectivamente). O comportamento do k. do $N$ form multo semelhantes, sendo que a diminuicaro da espessura da cobertura de ensofres elevadas temperaturas e precipitaça favoreceram a 1 iberaço doe nutrientes. Nas condicbes de trabalho dos autores Podzois arenosos, Eana-de achear, ensaio de campo, Fldrida-USA) $10 \%$ do $N$ pemmaneceu insal bivel por Varios anos. 
JAFFEL \& BOERSMA (1980) quantificaram e

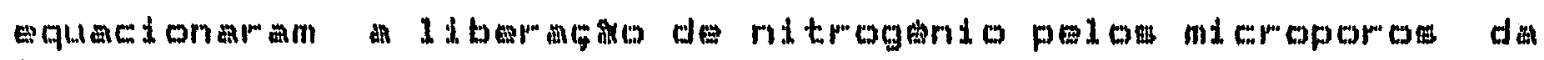
SCU, atraves de modelos matematicos. A liberaço depende principalmente do tamanho does poros na cobertura de enxofre e da temperatura do meio.

Num estudo realizado na Espantia com laranja PUCHADES et alii (1904b) observaram que gCU manteve teomes adequados de $N$ na soluço do solo mesmo nas epocas de Iixiviacao e perdas mais intensas, quando comparada a formas

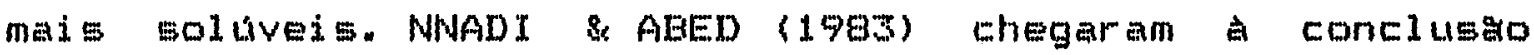
semelhante trabaliando com algodac e sorgo na Nigeria.

As perdas de nutrientes por lisiviara, principalmente de $N$ e ky sao muito inferiores nos FsL quando comparados as formas mais soluveis nas mais variadas condicBes KENGELSTAD \& FUSSEL, 1975; FUCHADES et alii, 1984a E CHITDLINA, 1978).

ATANASIU $(1559)$ Etudou a tax a de

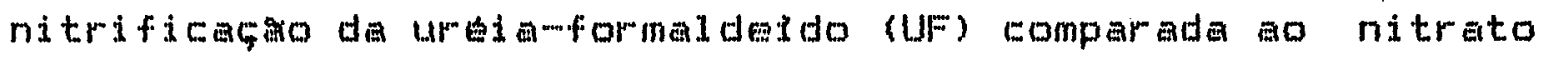
de amonio (NA), observando que duranke Lum ano a nitrifico da UF foi mais 1entan MATOCHA (1976) mediu a volati1izar da amoni em Podzdico distrofico textura media no TexasUSA, apos incorporaço de MA, SCW, ureia (U) Eulfato de amonio (SA). As perdas de $N$ por volatilizasoro foram 
inferioreg $1 \%$ no NA, SA SCU $13,5 \%$ na U. DALAL 19774 )

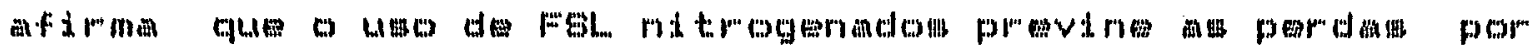
volatilizaça ou desnitrificaça.

NWADI \& ABED (1983) Dbservaram que a SCU năo altera o pH de solos com baixo poder de tamponamento na Nigeria. Observaca somelhante foi feita por DALAL (1975), comparando scu ureia. A ureia dimimuiu o pH do 5010 e aumeritou a quantidade de Al trocavel. A SCU nas afetou significativamente o pH ou o teor de Al trocável no solo.

No caso de gramineas, que permitem varios cortes sucessivom, quando comparamos FSL com fertilizantes

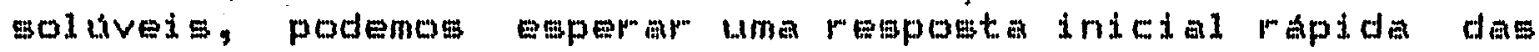
formes soluveis mefletindo en coltieitas iniciais maiores. Contudo as formas de Fish proporcionem, principalmente nos ultimos cortes, teores mai elevados de N nas plantas, coltheitas maiores major recupermbo final do $N$ aplicado (EEATON et al1i 1967 EOOONDUANG et alli, 1976).

Am doses elevadas de FSL aplicados de uma so vex nas afetam negativamente o desenvolvimento das plantas OU a germinacto das Eementes 1962). Trabalhando com trigo no campo na Belgica, testando diverma combinaçes de lumeiamaldeldo en comparaço com formas soliveis de nitrogenio, VERSTRAETEN \& LIVENS (1975) 
observaram que ต ineidencia de doences foi menor nos

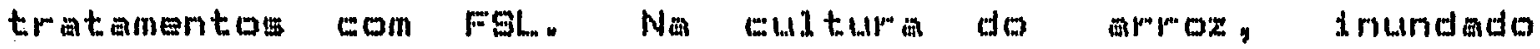
intermitantemente no Artansas-USA, WELLS \& SHOCKLEY (1975) obeervaram atraves da produca de graso, peno da materia sec e absor

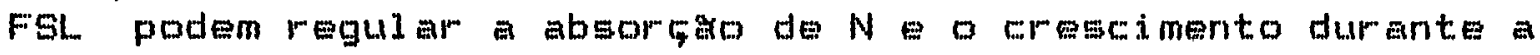
fase Vegetativa, As plantas dos tratamentos com FSL

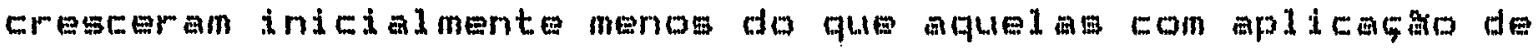
formas mais sol Livais.

WATSCHKE \& WADDINGTON (1974) relacionaram o teor de carboidratos livres na folha de gramineas com sua resistencia a dompase a calor e observaram que formas de FSL nitrogenadas nâso causam disturbios ou provocam comportanento diferente no teor de carboidratos livres en

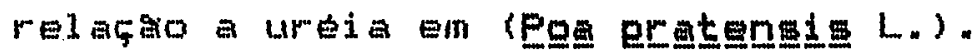

DAl..AL et alii (1974) num estudo realizado com milho una graminea formgeim em vasos comparou a absorcaro

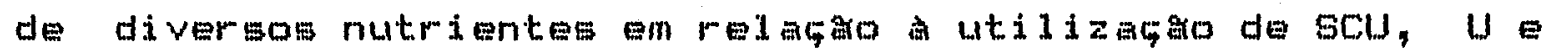
SA como fontes de Nitrogenio. O umo de scu fez com que as plantas absorvessem mais Kie Mg.

2.4. Manejo dos FSL

DE FSL sâto indicados para uso em condicBes 


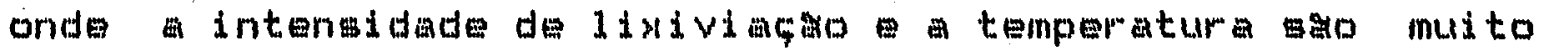

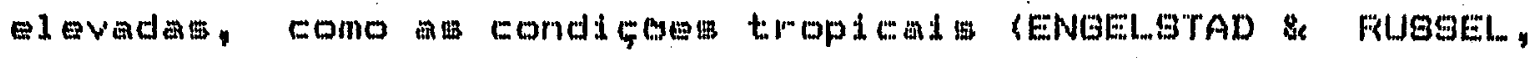
1975) ou 1 avouras inundadag como o arroz (PRASAD et ali $1971)$.

A utilizar fracionamento do fertilizante. WATSCHKE \& WADDINGTON (1974) observaram que uma aplitacto de frob equivalem a aplicaça continua de pequenas doses de urfia em graminea forrageira. A aplicaço Linica premplantio de SCU em tomate, na Carolina do Norte-USA, mubstitui cinco aplicaceses en cobertura de NA, suprindo toda a necessidade de $N$ da cultura (SHELTON, 1976 ). Na Nigeria, NNADI \& AEED (1983) constataram quE a aplicaら"a de scu produziu um resultado melhor do que duag cobertum a de nitrato de amonio e calcio (CAN) em somgo e algodao. A aplicacar lunica de fol nitmogenados em vamias gramineas produziu resultados semelinantes a aplicacaro semanal de NA (VOLK \& HOFN, 1975). Em Ema-de-achicar num Fodzol arenoso da F1drida-USA, GASCHO \& SNYDEF (1976) observaram que a sCu aplicada uma ver substitui quatro coberturas de sulfato de amonio (SA). Resultados semelhantes foram observados por SHAFMA et alii (1976) para uma gerie de hortalisas. SHELTON (1976) lembra que pelo fato do fSL no procisar ser fracionado a dose a ser aplicada anter do plantio é maior, sendo por isso mais fácil distribui-la eficiente e uniformemente na area. 
Na Eultura da I aranja na Espantia, MAQUIEIRA

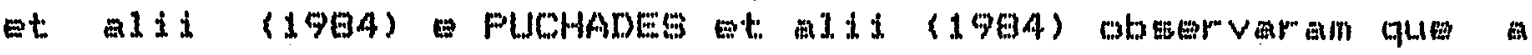
aplicaço de ScU na metade da dose de Luma forma mais soluvel - Eulfato de amonio nitrato (ANS) - atingiam produçes Equivalentes.

o nitrogento quando aplicado via FGL nato precisa necessariamente preceder imediatamente o plantio, a aplicaço pode ser feita lum bon tempo antes rSANCHEZ et a11i, 1973).

No caso de SCU e calcamio, ambos nao devem ser aplicados conjuntamente. Quando isso ororre aumenta a perda devido a volatilizacto do nitmogenio (MATOCHA, 1976).

Segundo DALAL (1975) a ap1icaço de SCU deve ser feita na superfitie, produzindo assim melhores resultados. A Limeia por outro lado deve ser incorporada a pelo menos $5 \mathrm{~cm}$ de profundidade.

Trabal hando com NA Envolto por uma membrana de resina LUNT \& OERTLI (1962) constataram que o teor de água no solo more o ponto de murcha permanente e a capacidade de campo naro afeta liberacalo do nitrogenio. A aplicacolo em cobertura, quando comparada a incorporacaso, diminui e metarda muito a 1 iberacåo. 
LIEGEL \& WALSH (1976) trabalhando com mitho

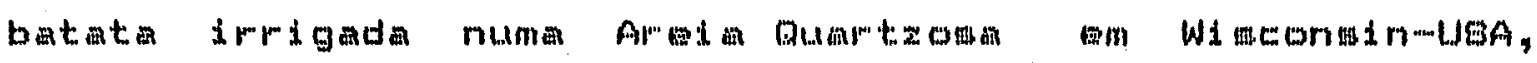
constataram que a SCU quando aplicada numa porfaro do solo que sempre permanece Limida sua eficiencia aumenta muito.

\subsection{Resultados experimentaim}

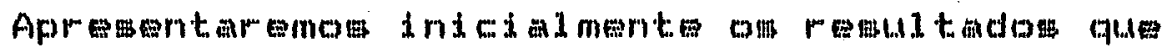
atribuiran alguma vantagem pela lutilizaça de FSL posteriormente aqueles ein que os resultados foran semeltiantes ou inferiores em relabar as fontes mais soluveis.

\subsubsection{Result adom favormvalm}

DALAL (1975) trabalhou com milho em Trinidad, num solo acido de testura media numa epoca chuvosa. Utilizou SCU $2 U$ e constatou que en todos os tratamentos a sCu se comportou melhor, levando a mai or produtividade das plantas. Fesultados semel hantes form obtidos para a cultura do milho por DALAL $(1974)$ E FFYE (1977).

KHANDELWAL et: ali (1977), obteve maiores productes de trigo com ureia coberta por deo de (Axadirachta indlea), tanto de palha como de grags, do que com ureia. 
Citrum tiveram protuçes maioreg e com doess

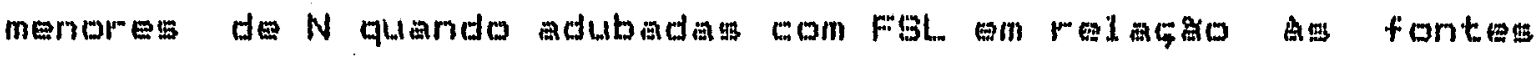
mais SolLVeis (PUCHADES et alii, 1984b).

A producto de batata, aveia e trigo foi maior no campo en tasa de vegetacto quando foi utilizada a combinaço ureia formaldeido em relacko a NA SATANASIU, 1959).

Diverma gramineas forrageimas responder am

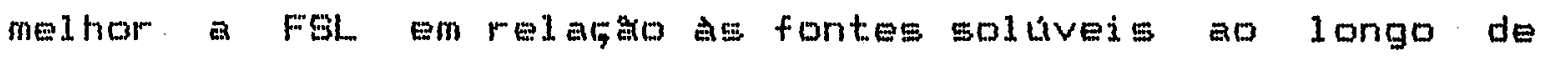
cortes suressivos en ensaios conduzidos por EOONDUANG et a1ii (1976) POr WADDINGTON \& DUICH (1976).

Nas culturas de agodaro e sorgo na Nigeria, NNADI \& AEED (17\%3) obtiver"am melhoreg pesultados com fontes nitrogenadie de FSL comparydas as fontes mais soluveis.

SANCHEz et ali (1973) trabalhando com arroz

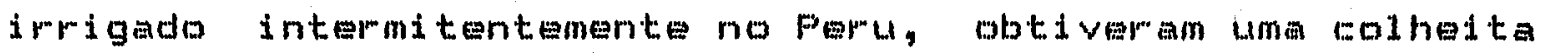
$59 \%$ superior crom SCU em rel acto a U SA.

A producto a qualidade de hortalicas (SHARMA et alii, 1976) eplantas ormamentais (SCOTT et alii,

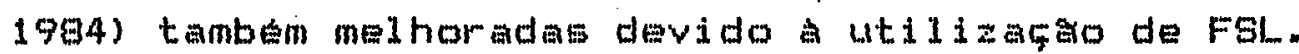


LIEGEL \& WALSH (1976) encontraram um desempentio melhor do mitho da batatintia imrigada da sou em relaço a $U$, so quando no houve coberturas no tratamento com $u \in$ quando a Intensidade de livivimgaro foi muito internsan.

TEFMAN \& MORTVEDT (1979) trabalhando di versos tipos de FSL nitrogenados e NA com milho e arroz inumdado em Vasos alertam par of ato de elevolas doses de p lmais elevadas do que em condicbes de campol terem que ser usadas parma avaliar qual quer fonta de $N$ ou kg senao esse nutriente se torma limitante nao ha diferencas entre as fontes.

\subsubsection{Rom}

VAUGHN et ali (1979) aplicamam Ureiay. Ureia + Ensofre em po e SCU numa pastagen natumal na CaliforniaUSA E estudaran o efeito da aplicaço durante cinco anos. Constatamam que a SCU se comportou de maneira eneltiante a

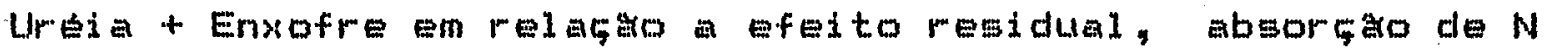

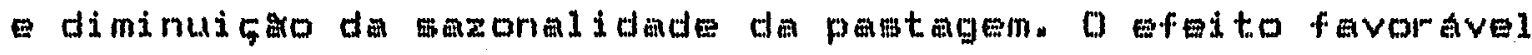
desses dois tratamentos em relaco a ureia foi atribuido a presenca do ensofre e no a cobertura com ensofre. 
TEFMAN et ali i (1970) compararam superfosfato

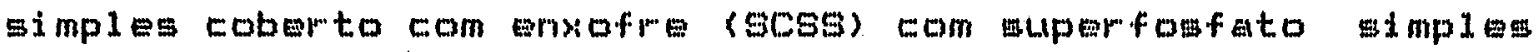
(55) em arroz inundado num ensajo em vasos. Constataram que - SCSS nao ficou dimponivel as plantag no primeiro ano de cultivo que as formas mais soluveis de fosforo produzem resultados melhores.

EEAUCHAMF (1577) Estudow a adubaga, do milho no outono eplantio na primavera de FSL nitrogenados en comparasăo a ureia. Nä foi observado nenhum efeito residual dos FSL m melaço a Lreia, naso havendo vantagem na produca pela aplicacas de FSL no outoncs ao inves da Lreia.

GILLIAM at alid (1993) compararm a mitubaco d : fertilizantes fluidos nă encontraram diferenca na qualidade ou na producáa das plantas.

A produca do feno de diversas mepecies no Colorado-USA, en. tres Molissois a grande altura capron. 2. $400 \mathrm{~m}$ NM) nato fol aumentada pelo uso de FGL nitrogenados, nơ Eendo detectado efeito mesclual dessas fontes.

HAAN \& WEFKHDVEN (1975) EStLdaram 20 produtos da condensaço da ureia com algum aldeido em ensaios de incubacto por 16 semanas. Os produtos 
apresentaram um comportamento muito variado e foran menos

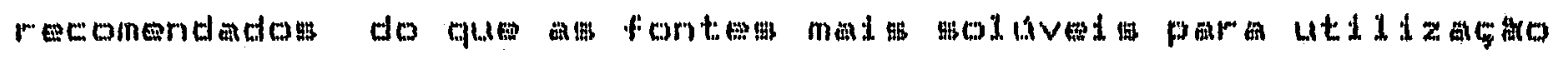
na agricul tura. 
3. MATERTAL. E METODOS

\section{3.i. Suporte capilar para fertilizantes flufdos (SC) e seu preparo}

$0 \mathrm{sc}$ a bastcamente um elindro de gesso (Caso noH o) compotado. Esse cilindro 6 imerso numa A 2

solusto, contendo fertilizantes fluidos Eses solucto fira Petida no interior do $5 \%$.

3. 1. i u Preparo do sc nos ensaios de efeito residual e de micronutrientes

No caso desses dols ensaios fol utilizado 1

gesso agricola e oproduto final, sem ter absorvido a

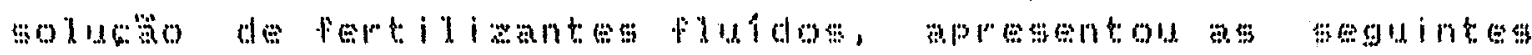
caracteristicas:

10 gesso agricola e um gesso resicidal (caso. 24 o) subproduto da producto de acido fosporico. 
- $6,0 \mathrm{~cm}$ de comprimento

"2, com de dimetro

- 14,0 g de massa

" $84 \%$ de poros ou $31 \mathrm{~cm}^{3}$ por $5 \mathrm{C}$

A mapeidade de absorver mme solucäo cristalina foi da ordem de 13,0 g. proborouse a seguir

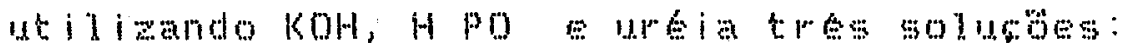
34

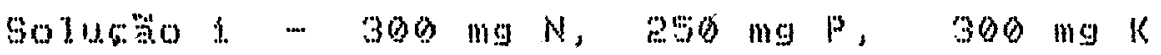
por $\quad 139$ de cormespondents a nivel it

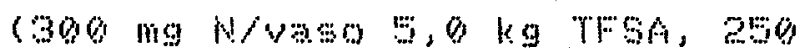
mg P/Waso $6,0 \mathrm{~kg}$ TFSA, $300 \mathrm{mg}$

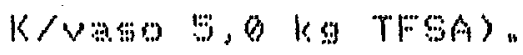

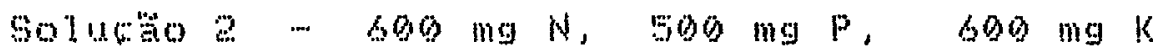

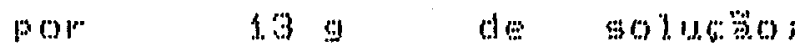
correpondente a nlvel 2 $600 \mathrm{mg} N / \mathrm{Na} 5 \mathrm{~B}, 0 \mathrm{~kg}$ TFSA, 500 mg PrVAso $8,0 \mathrm{~kg}$ TFSA, $600 \mathrm{mg}$

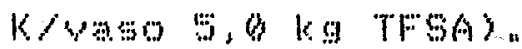

$601460303-900 \mathrm{mg}, 790 \mathrm{mg}, 900 \mathrm{mg} k$ por do do \$oldo: correspondentio no nivel 3

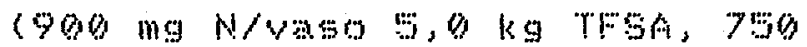
mg P/Vaso $5,0 \mathrm{~kg} \mathrm{TFSA,} 909 \mathrm{mg}$

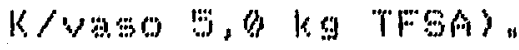


Og SCa for am imersos nessas solucbes, ate

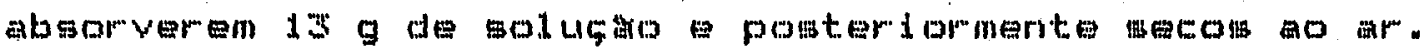

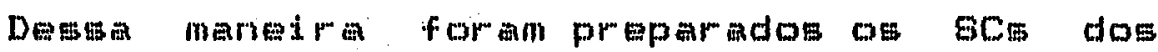
niveis $0,1,2$ e 3 do ensaio de efeito mesidual.

No ensaio de micronutrientes procedeu-se de marreira semelharite para o preparo dog SCs. Nos SCg que contintram micronutrientes utilizoume os seguintes compostos

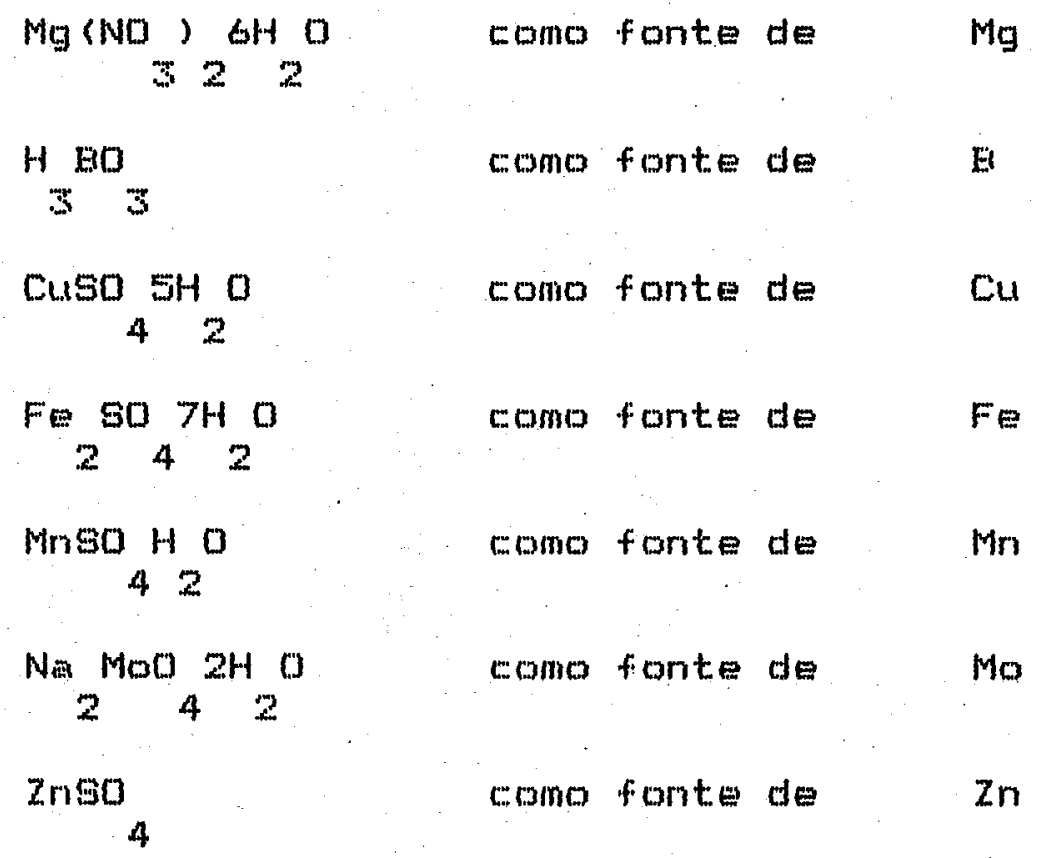

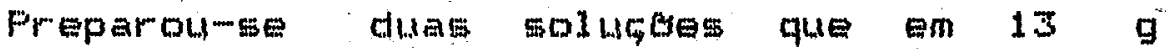
corresponderam aos niveis finais seguintes: 


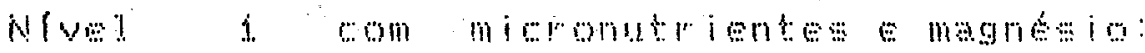
it

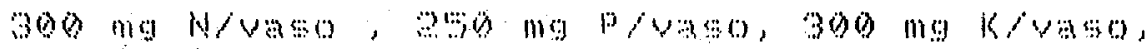

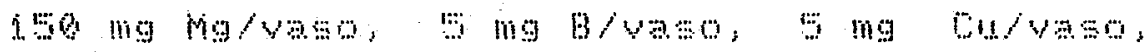

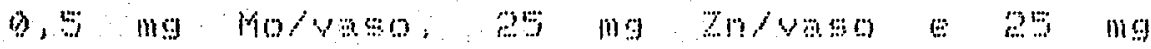
Fin:

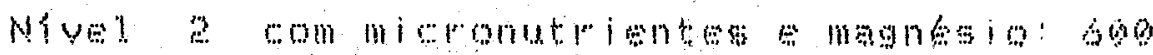
婂 mo N/y so,

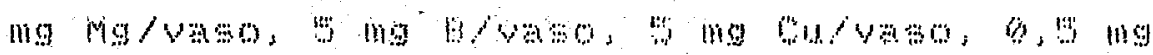
Hovaso, 25 mg zn/yso e 25 mg Fe/vaso.

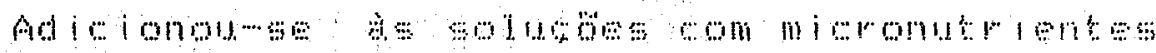

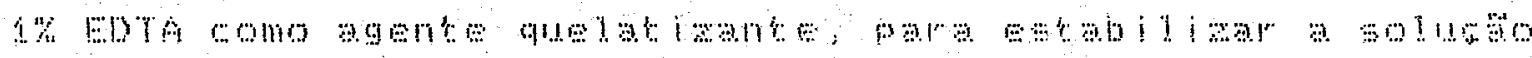

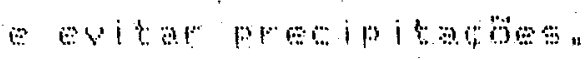

\section{1. 2. Preparo do oc no ensato de liniviato}

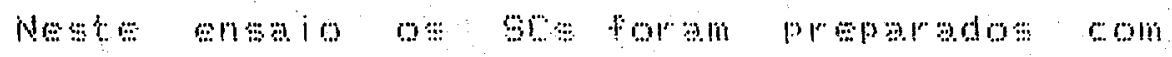

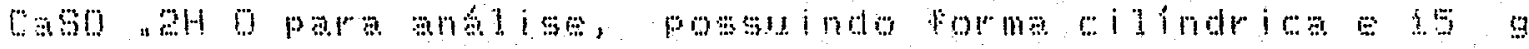
42

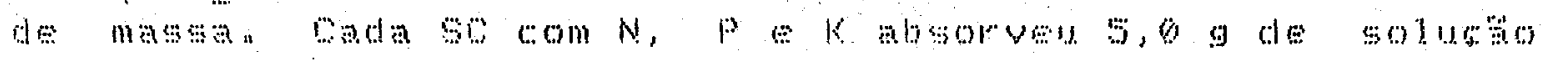

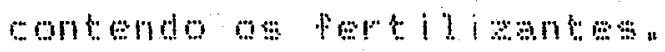

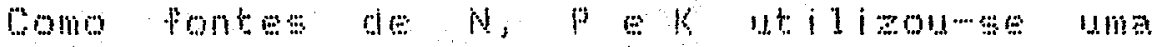

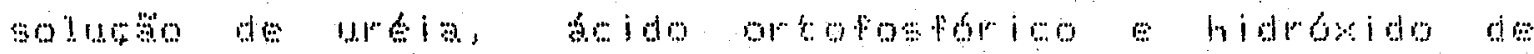

it:

40:50\% 
potassio mespectivanente. A mesmin eoluço foi utilizada como fonte de fertilizantessoluveds (Fis).

A dose utidizadem todos os tratamertos que receberam adico de fertilizantes foi de 46 ppm de ky 23 ppm de $P$ ers pom de $N_{n}$

\subsection{Ensaios realizados}

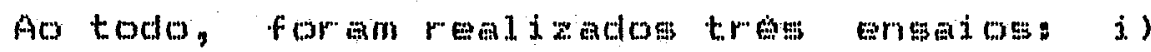
ensaio de efeito residual, iis ensaio de micronutrientese

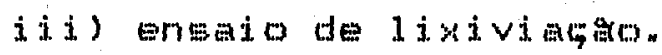

-. Ensaio de efedto restdual. os fertilizantes de solubilizacoro lenta (Fols) apresentem, quando comparados a

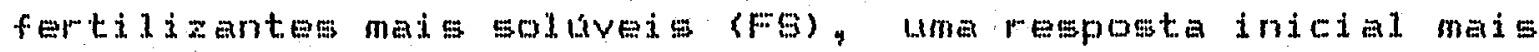
lenta. Se considerarmos entretanto, wultivos ou cortes sucessivos esperawise que ao 1 ongo do tempo os FSL mantenham

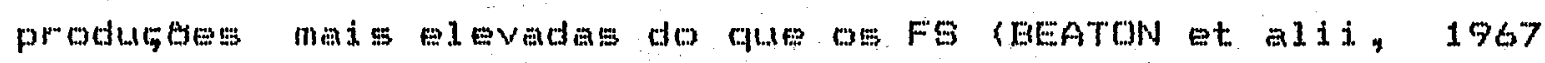
E AOONDUANG Et a it, 1976).

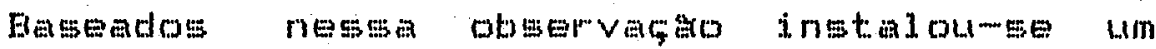
ensaio em vasos, utilizando capim Coloniao ev tobiata (Eanicum maximum cv tobiata) como planta teste, procedendose quatro cortes mucessivos. Ainda nesse ensaio, utilizou-se tratamentos com sem calagem, dois tipos de solos e quatro 
doeses de $N, F E K$

Des tratamentos básicos consistimam de luso de

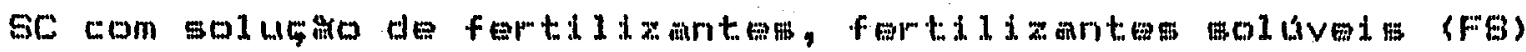
misturadom ac solo e gC mm soluga de fertilizantes laperias o Eilindro de gemol + aplicacaro de Fis misturadom a

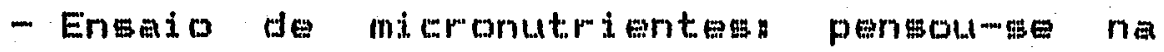
aplicaco de mitronutrientes via SC, isto e incluidos na solucăo de fertilizantes fluldos que é absorvida e armazenada dentro do SC.

A fim de avalim am possibilidade,

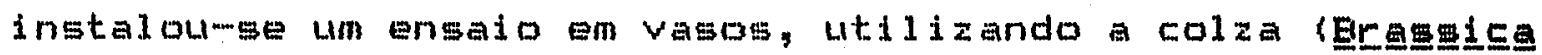

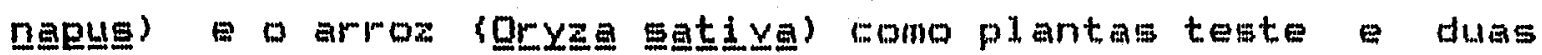
doses de N, F K K

De tratamentosi basicos consistiram de:

* SC con micro - SC armazenando soluca do de fertilizantes fluidos $N_{y} P$ e K + micronutrientes:

* SC micro no \$olo -- SC armazenando 5ol 45 สัo de fertilizartes fluidos $N_{4} F E K E$ OS micronutrientes mistumados ao sol o:

* SC sen micro - SC armazenando solusado de fertilizantes fludos $N, P$ e $k$ sem aplicatoro de 
micronutrientes:

* FS + GC mimo no solo -.. BC sem fertilizantes (apenas o cilindro de gesso) + fartilizantes He at a Eolo

* FS + SC sem micro - GC Eem fertilizantes + FS misturados wo solo sem aplicaço de microriutrientes.

- Ensaio de lixiviagar a fim de avaliar a Iixiviagaro de nutrientes do SC instaloumse um ensaio em colunas de solo. Provocoumbe a lixiviaga adicionando-se agua às colunas.

OE tratamentos basicom consistiram: SC armazenando soluço de fertilizantes fluidos $N, P$ e K, SC

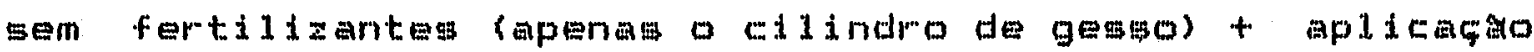
de fertilizantes de alta golubilidade (FS), FS e uma testemuntia.

3.2.1. Ensaio de efeito pesidual

3.2.1.1. Tratimentom basicom

Os tratamentos basicos for am os seguintes:

i) Suporte Capilar com Fertilizantes Fluidos- 
SC com; ii) Fertilizantes de flta solubilidade wFS

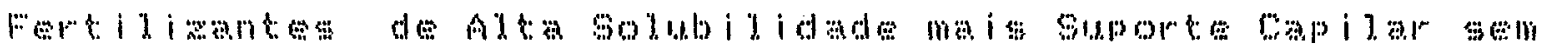
Fertilizantes Fluidos Fs +. FC gem.

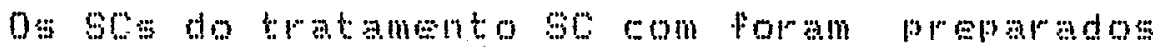

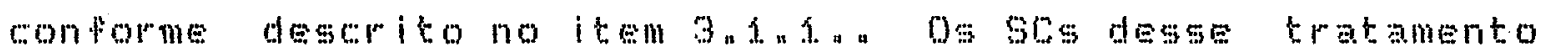

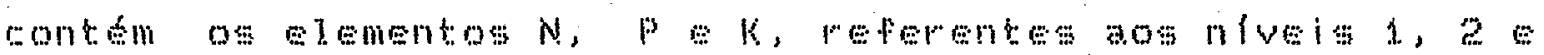
3 armazonados nos seus poros"

No tratamento FS, Pertiliantes de alta solubilidade, ut l i

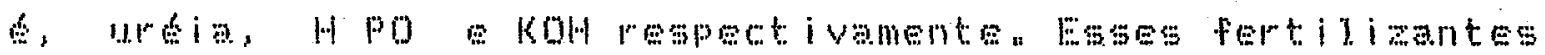
34

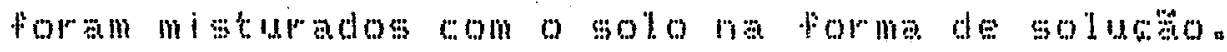

No tratamento FS + SC sem procedause da mesma maneira do ane no tratamento lis, isto foi misturado com o solo em soluson Alsm disso, foi

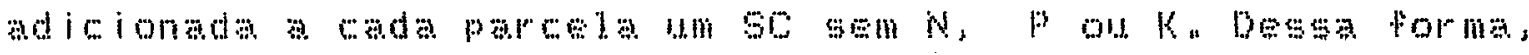

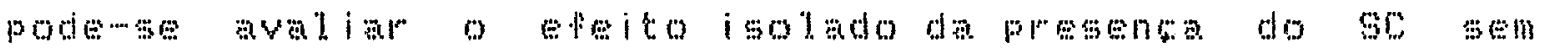

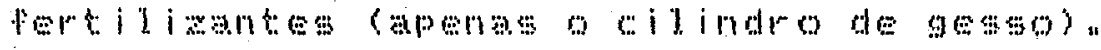

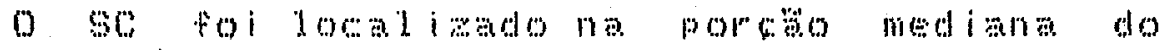

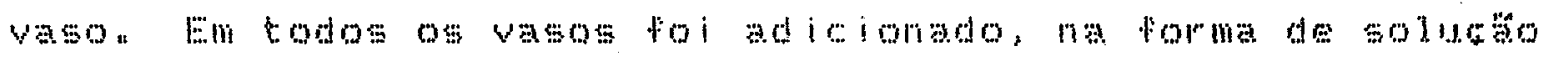

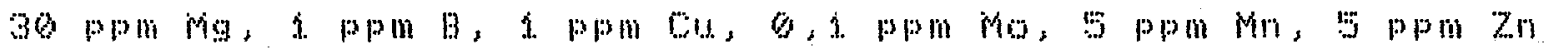
e 


\subsubsection{Domes de $N, P \in K$}

As dosagens utilizadas nos niveis $0,1,2$ e 3 estao representadas a seguir:

\section{Nivel}

()

1

2

3

Dose. PPm

$\begin{array}{lllll}N & 0 & 60 & 120 & 180 \\ K & 0 & 50 & 100 & 150 \\ K & 0 & 60 & 120 & 180\end{array}$

Naro foi utilizada adubaça em cobertura ou qual quer outro tipo de adicta de $N_{4} F$ ou K apos a instal aço do ensia.

\subsubsection{2... Solo:}

For motidizactom dol molos: i) Latossolo Vermeltio Escuro distrofico, A moder ado, textura media - LE e i1) Latossolo Roxo distrdfico, A moderado - LF.

Oss dol molos form coletados no Municipio de 
Piracicaba, utilizando-se a camada de 0 a $30 \mathrm{~cm}$.

Analises quimicas para fins de fertilidade foram realizatas e os resultados pota popresentados a Eeguir:

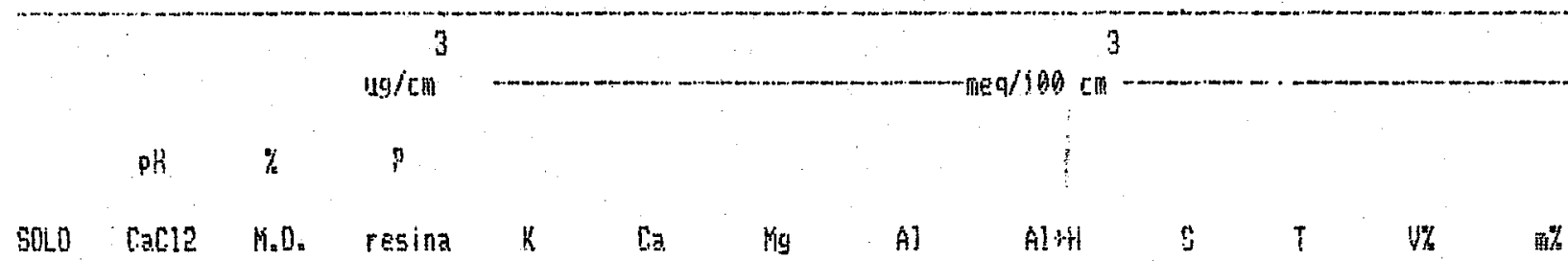

$\begin{array}{llllllllllllllll}\text { LE } & 4,4 & 1,6 & 12,6 & 0,17 & 0,50 & 0,22 & 0,48 & 2,77 & 0,97 & 3,74 & 24 & 33 \\ \text { LR } & 4,4 & 2,7 & 34,2 & 0,54 & 0,96 & 0,43 & 1,19 & 7,16 & 1,95 & 9,41 & 24 & 36\end{array}$

o. terra foi destorroado e peneirado com peneira de $2,0 \mathrm{~mm}$. A terra foi acondiciunada em vasos de cerámica impermeatilizados internamente con "neutrol". Em cada $v a n g$ formo colocadom (TFSA).

Fara todos os tratamentos basicos e para os doi solos foran utilizadom tratamentos com e sem calagen. 


\subsubsection{Calmgem}

Nos tratamentos com cal agem, a aplicaça do

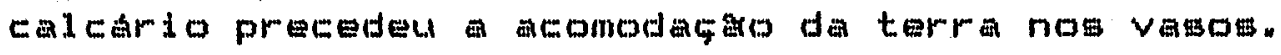

Utilizou calcario comercial da regiơ, tendo o mesmo as seguinteg caracter isticas:

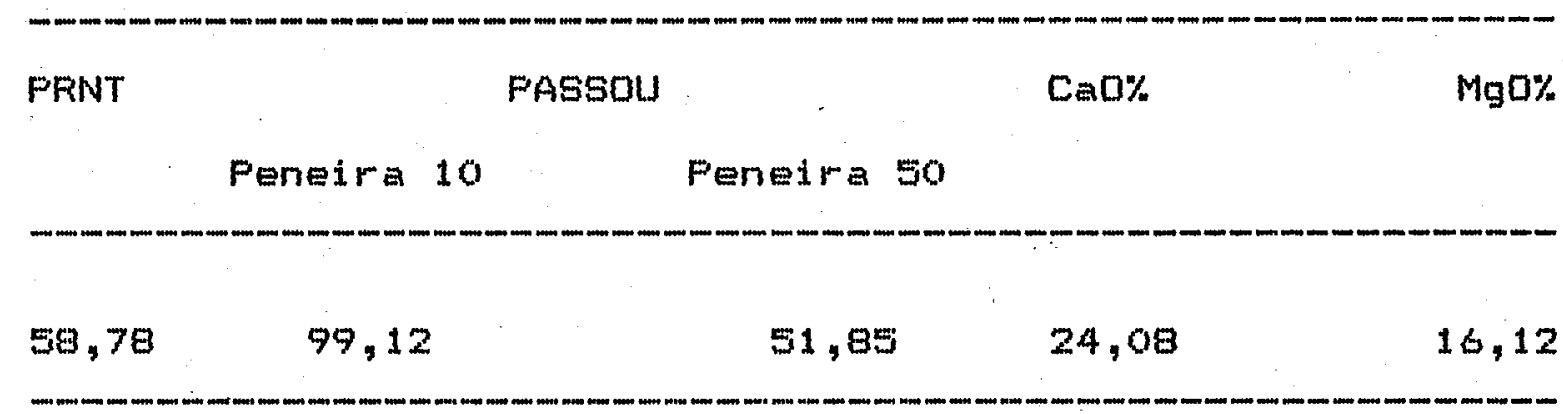

Estimoumse a quantidade necessaria para elevar a saturacono por bases a 70\%. A terra, apos peneirada, foi misturada com o calcamio e mantida proximo a capacidade de campo por bo dias. Ao temino desse perlodo, nova amostra foi coletada e submetida a andise qutmicapara fins de fertilidade, On mesultados; apos a calagem gă apresentados a seguir: 


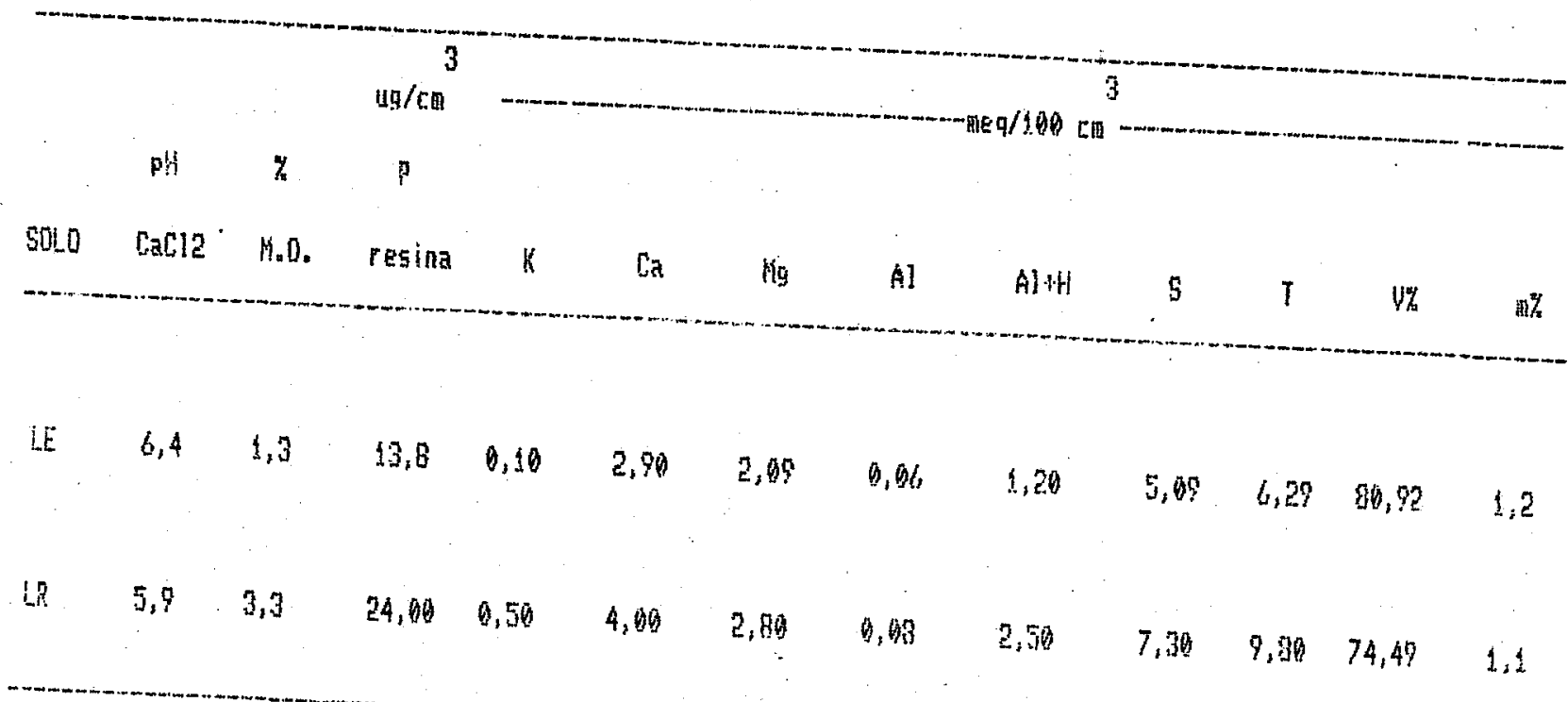

\subsubsection{Cultura}

A cultura vegetal utilizada foi o Capim Coloniago cultivar Tobiatio (Eqnicun maximun cv. Tobiata); escolnido devido a sua alta exigencia pomibilidade de efetuar cortes suceseivos. Foram plantadas 5 mudas de 10 di as en cada $V$ a desbaste deixando apenas duas plantas em cada vaso. 


\section{2uin Cortes}

0. cortes do canim foram feitos a 5 com de

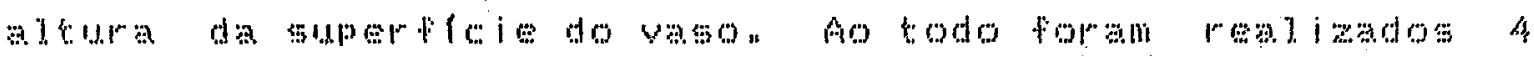

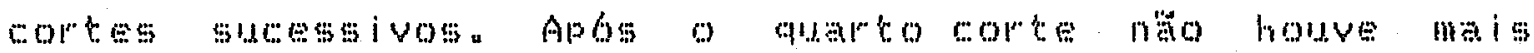

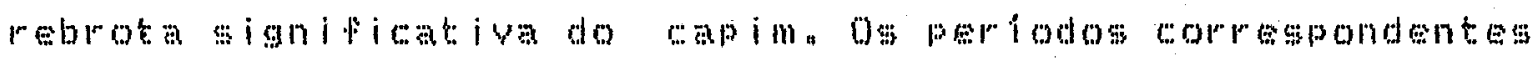
aos autro cortes foram os sogunts:

\begin{tabular}{|c|c|c|c|c|}
\hline$\hat{\mathbb{1}}$ & corte & $0101 / 02$ & ?] & $12 / 03 / 86$ \\
\hline 2 & corts & $12 / 03$ & $\pi$ & $16 / 04 / 86$ \\
\hline 3 & : & 10104 & $a$ & $20 / 06 / 86$ \\
\hline 4 & $\cdots o l^{n+\theta}$ & $20 / 06$ & 至 & $27 / 00 / 86$ \\
\hline
\end{tabular}

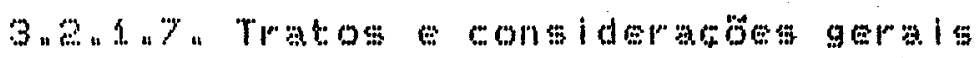

o: vasos form rolocados mobre mesas de madeira de $1,30 \mathrm{~m}$ de alturn, en cima depratos de plostico,

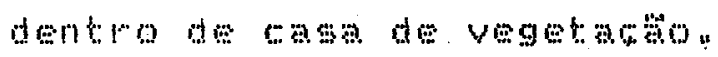

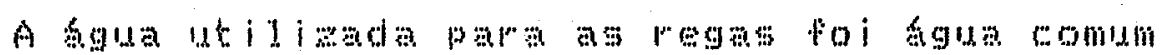
de torneira, sendo os vasos irrigados pelo menos uma vez por

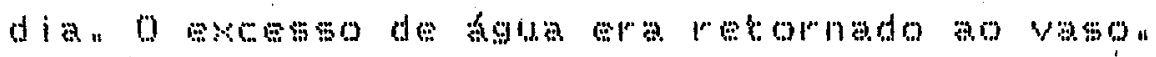

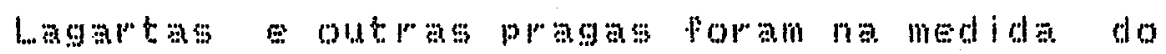

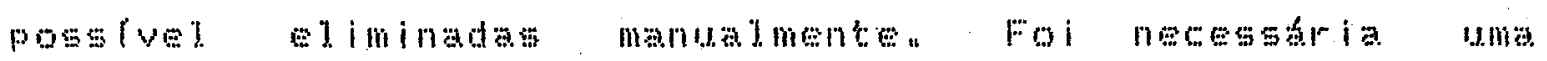

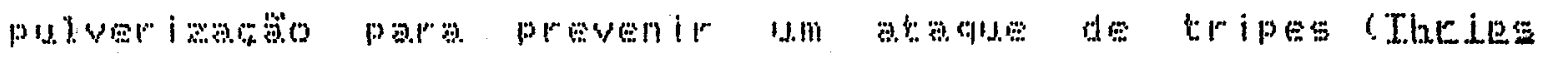




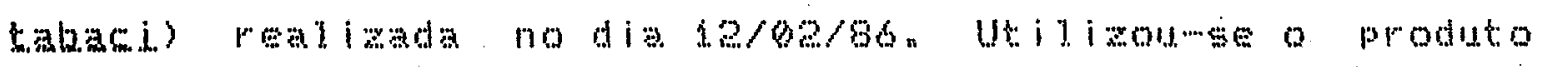

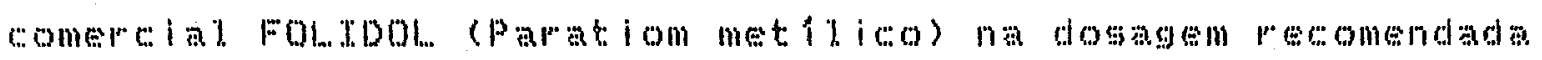
pelo fabricante.

No din $20 / 02 / 86$, isto te, na metade do periodo do primilo corte, as plantas comeraran a evidenciar um

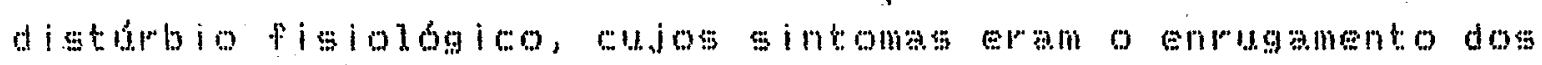
bordos das folhas, semelhange dua deficiencia de zn. Foi

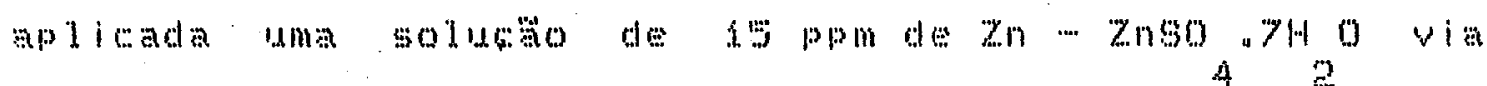

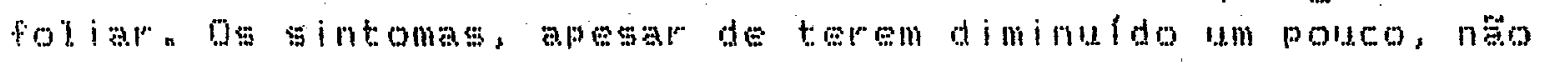

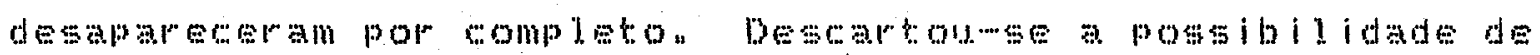

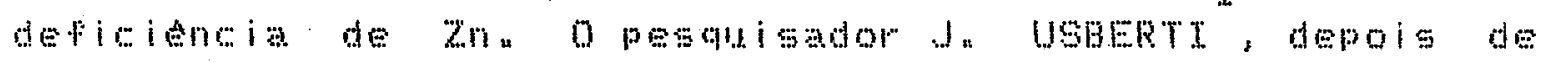

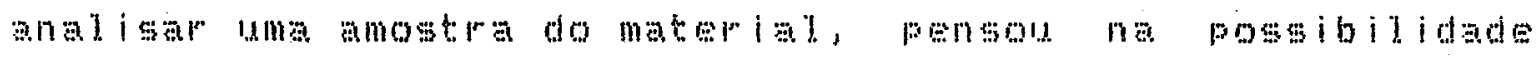

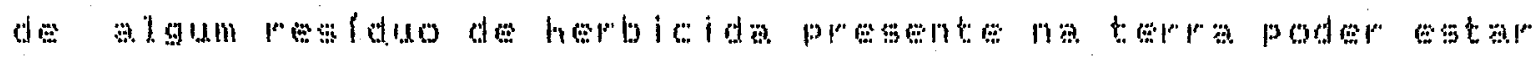
casundo o distoblo

A Him de anmentar a atividade biologica dos mioroorganismos na serra do vaso e, onsenentemente, degradar rabiamente qual quer residuo do herbicida presente,

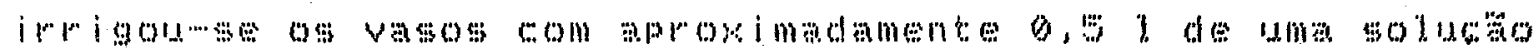

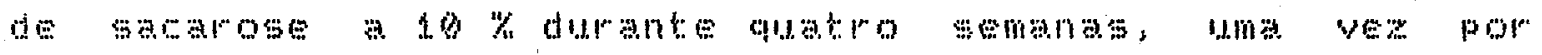
semana. Logo apos a primeira irrigato os sintomas diminuiram sensivelmente, desoparecendo por rompleto apos

1.

USBERTT, Jn (Instituto Agronomico do Estado de gao Paulo

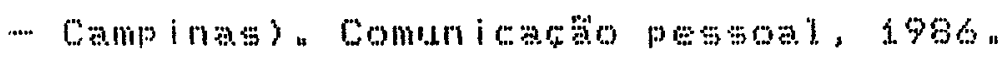


quarta semana ná voltando a manifestar. Consideramos

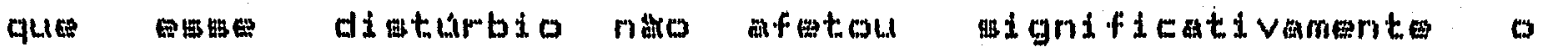
comportamento das plantas, năo comprometendo os resultados.

Oes Vamos, dentro de cada bloco, foram casualizados repedidas vezes durante o ensain.

3.2.1.8. Delineamento experimental

o ensaio foi instalado em Elocos Inteiramente Casualizados con 3 Tratamentos Easicos $* 2$ Niveis de Calagem : 2 Solos $x 4$ NIVeig de N, F $K x$ S Repeticoes $x 4$ Eporas de Colheita (Sub-rarcel as) de modo fatorial. o total de tratamentos $\& 48$, totalizando 144 parcelas e 576 sub-

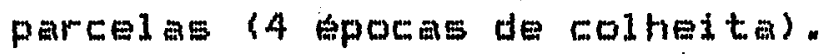

3.2.2. Ensaio com micronutrientes

3.2.2.1. Tratmentom bamicom

QB tratamentog basicos form $Q$ a meguintes:

1) Suporte Capilar com Fertilizantes NFK com Micronutrientes - $5 \mathrm{SC}$ com mieroł iis Suporte Capilar com Fertilizantes NFK e aplicacto dos Micronutrientes no $\$ 010$ sc micro no solos iii) Suporte Capilar com Fertilizantes 
NFK sem aplicaço de Micronutrientes - SC sem microi iv)

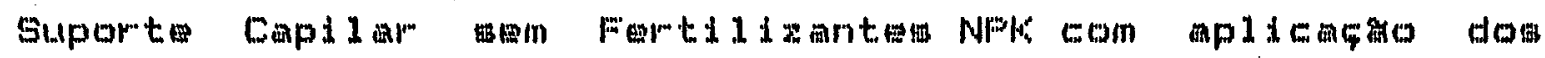
Fertilizantes de Alta Solubilidade no solo e aplicaça dos Micronutrientes no solo - FG + SC micro no molo e v) Suporte Capilar sen Fertilizantes NPK com aplicaçào dos Fertilizantes de Alta Solubilidade no solo e sem aplicacao dos Micronutrientes - FS + SC sem micro.

Q SCS foram preparados conforme o item 3.1.1. No tratamento SC com micro os fertilizantes $N, F, k$ e os micronutrientes foram IoCal1zados no interior dos SCs, absorvidos de uma solucäo.

No tratamento SC micro no solo, apenas os fertilizantes $N, P \in K$ foram aplicados no interior dos SCs. Qs micronutrientes foram aplicados ao solo, atraves de uma soluço que foi misturada com a terra.

No tratamento SC sem micro os fertilizantes

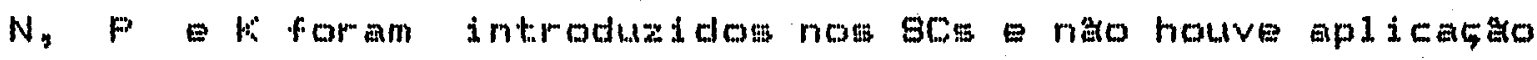
adicional de micronutrientes no solo ou pelo SC.

As parcelas do tratamento FS + SC micro no solo receberam a aplicaço dos fertilizantes de alta solubilidade no solo, migturando-se a terra com uma solucaro contendo $\mathrm{KOH}_{4} \mathrm{HPO}_{4}$ e urei- $\mathrm{Os}$ micronutrientes foram 
misturados com a terra da mesma maneira. Ds sCs utilizados nesse tratamento nac contintion nentum tipo de foutilizante.

No tratamento FS + SC sem micro procedeu-se da mesma maneira descrita no caso anterior, apenas omitindoBe a apicaco dos mitronutrientes no 5010.

Q5: SCE foram tambem localizados na porfá mediana dos vasos.

Procedeu-se dols cultivos sucessivos. A

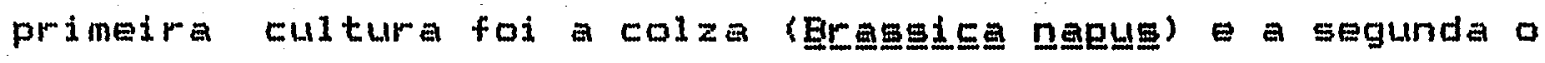
arroz (gryza sitiva).

\subsubsection{DOBEm}

As doses lutilizadas corresponderam aos niveis 1 2 2 do ensaio de efuito pesidual (item 3.2.1.2.), conforme esquema abaixo:

N

$$
p \quad F \quad m
$$

$\begin{array}{cccc}\text { Nivel } 1 & 60 & 50 & 60 \\ \text { Nivel } 2 & 120 & 100 & 120\end{array}$


Ein todos os tratanentos com aplicacas de

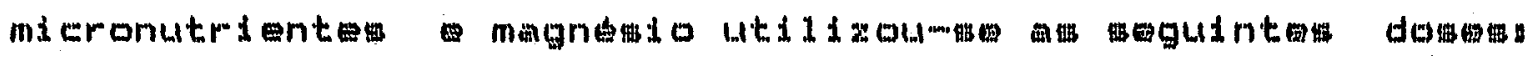
Mg 30 ppm, E 1 ppm, Cu 1 ppm, Fe 10 ppm, Mn 5 ppm, Mo 0,1 ppme $\mathrm{Zn}=\mathrm{ppm}$.

No segundo cultivo (arroz) reaplicou-se a metade da dose de $N, F, K$ em todos os tratamentos sem contudo reapliear oes micuronutrientes. Desom forma, forain reaplicados no plantio do arroz no nivel 1: 30 ppm de $N, 25$ ppm de Fe 30 ppin de Ke no nivel 2.60 ppm de $N, 50$ ppm de F e so ppm de k. Essa meia dose foi aplicada da mesma forma do. tratamento, isto na forma de fertilizantes de alta solubilidade nos tratamentos FS + SC micmo no solo Fs + SC sem micro armaznados nos SCe nos tratamentos $8 C$ com micro, SC micro no solo e SC sem micro.

\subsubsection{5010}

o solo utilizado foi uma Areia Ruartzosa; alica, A moderado

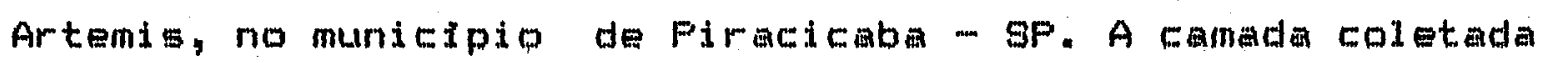
由 de 0 a $30 \mathrm{~cm} d \pm$ profundidade.

A ankijse quimica para fins de fertilidade rovelou os seguintes resultados: 


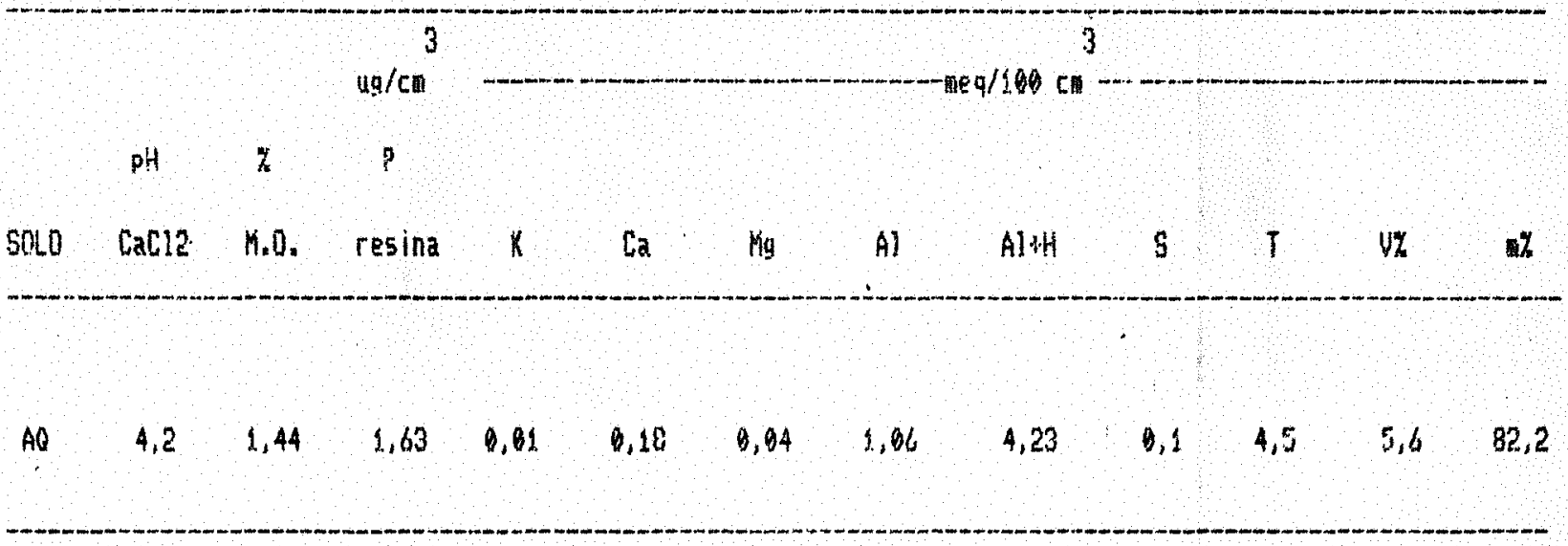

0 terra tol destorroada e peneirada com peneira de $2.0 \mathrm{~mm}$. A terra fol acondicionada em vasos de ceramica impermeab 11 izados com "neutrol". Foram ut 11 izados 5, o kg de terra fina seca ao ar por vaso.

\section{$3.2,2,4, C a 1$ agem}

A calagem fol realizada em todos os tratamentos. o calcario utilizado fol o mesmo do ensalo de efeito residual (item 3.2.1.4.). A quantidade de celeario fol estimada para elevar a saturacapor bases a $70 \%$ \% 
Misturou-me toda term com o calcario foi mantida a Capacidade de Eampo por $60 \mathrm{di}$ ă.

\subsubsection{Cul turm}

Realizou-se dois, cultivos sucessivos. No primeiro pertodo, de 15/06/96 a 27/08/86 optou-se pelo

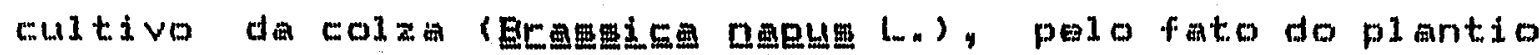
ter sido realizado numa epoca maja fria do ano e esta planta adaptar-se melhor a essas condicbes. O plantio foi feito por mudas, transferindo-se o plantas para cada vaso que foram posteriorinente desbastadas, permanecendo apenas duas.

No segundo periodo de cultivo optou-se pelo arroz (ㅁy르 Sattivaㅡ L.) variedade IAC 165. D periodo mais quente do ano e mais favoravel ao desenvolvimento dessa especie. o plantio foi realizado no dia $15 / 10 / 86$ a a colheita no dia 20/01/87. Foram utilizadas mudas, sendo a popul aço final de 5 plantas por vaso.

3.2.2.6. Colheita

Os cortes foram realizados na colza e no arroz quando a maioria das plantas ja havia florescido, removendo-se toda a parte aerea das plantas. 
Qw periodos commepondentes a cada cultivo foram ons guintes

$\begin{array}{ll}\text { Colzin } & 15 / 06 / 86 \text { a } 27 / 09 / 96 \text { (colhedta) } \\ \text { Arroz } & 15 / 10 / 86 \text { a } 20 / 01 / 97 \text { (colheita) }\end{array}$

3.2.2.7. Tratos e consideragBes gerais

DE vasos foran colocados sobre mesas de madeima de 1,30 $\mathrm{m}$ de altura, en cima de pratos plasticose mantidos ein casa de vegetaço.

Fara as regas utilizoumse agua destilada, sendo os vasos irrigados pelo menos uma vez por dia. Quando havia exiesso de agua a mesma er retornacta ao vaso.

Naro houve necessidade de controle de pragas ou doencas.

DI Vasos for am camalizados mepetidas vezes dumante o trangcormer do ensiojo.

\subsubsection{Del i neamento experimental}

0 ensaio foi instalado en Elocos Inteiramente Casualizados com 5 Tratamentos Basicos $x$ 2 Niveis de 
N. $P$ E $x 4$ Repetifema de modo fatorial. o totai de

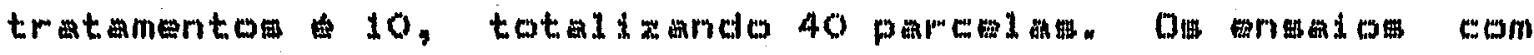
colza e arroz form analisados separadamente.

\subsubsection{Ensaio de Iixiviaca}

3.2.3.1. Tratamentom bats

En colunas de terra provocou-se a 1ixivi aça nas sequintes condi

a) Suporte Capilar Para Fertilizantes Fluidos (SC) $\operatorname{com} N, F E K-S C$ cam;

b) Suporte Capilar para Fertilizantes Fluidos (sc) sen $N_{4} F \in k+F e r t i l i z a n t e s$ de Alta Solubilidade (FS) como fontes de $N, P$ e $K-S C$ sem

E) Fertilizantes de Alta Solubilidade como fonte de $N, P E K-F B E$

d) Testemuntia, sem aplicaca de $N, P \in K:-$ TEST.

Do SC form preparados confomm descmito no item 3.1 .2$. 
0 solo utilizado foi o Latossolo Vermelto Escuro, distrofico textura media, A moderado, do muricipto de Jaboticabal. 0 solo se encontrava sob uma mata de Eucalipto bastante antiga (apros. 10 anos) y no tendo sofrido correfaco ou adubacto durante esse periodo. A camada coletada de 20 a $40 \mathrm{~cm}$. Escolhel evitar a CTC mas elevada da superficie, ocasionada pela presenca de materia orgunica e pelo fato da lisiviacio ger un fenomeno que ocorre en profundidade.

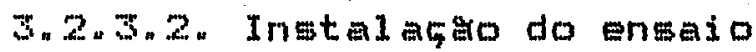

As colunas de terral for am montadas a partir de sete gegnentos de $10 \mathrm{~cm}$ de 1 tura de cano de FVC com 14,4 cm de di ametro interno.

o volume de Terra Fina Sera ao Ar (TFSA) adicionado a cada coluna foj de $11.400,2 \mathrm{~cm}$ a massa dessa terra compeponde a $15.012,9$. Dessa melacio chegamos a um valor de $1,32 \mathrm{~g} / \mathrm{cm}$ de densidade da terra dentro das colunas. Como a densidacte das particulas desse solo e de 3

2,34 g/cm (determinadia pelo metodo do alcooj) chegamos a um porcentagem de poros de $43,72 \%$ em cada colura con sete segmentos de $10 \mathrm{~mm}$ cada. 0 volume total de poros e de 4. $984 \mathrm{~cm}$ em corda colume. 
Os: 50 OS FS form posicionados na parte

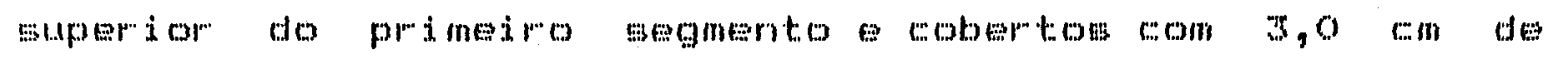
terrat.

Cida coluna foi mepetida quatro vezes sendo o delineamento estatistico montado em Elocos Inteiramente Casuedizados com 4 Tratimentos Easicos $x 4$ Fepeticoes $x 7$

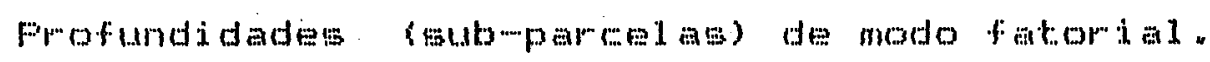

Antes de iniciar o ensaio as colunas foram 1. puadas a caparidade de compo.

\subsection{3.... Condumo do masio}

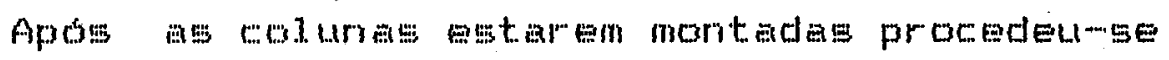
o inioio das lixivimbBes, utilizando agua destilada $A$ primeira Iixiviacoso foj foita com 2,50 de dqua por coluna iadicionados $20 \quad 10 \times 2 m 0 \mathrm{ml}$ com 15 minutos de intervalo entre cada nova adicao). Com essa quantidade de mgua as

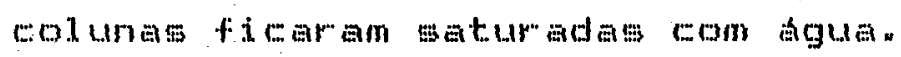

Duas semanas apos, inicioum novo ticlo de

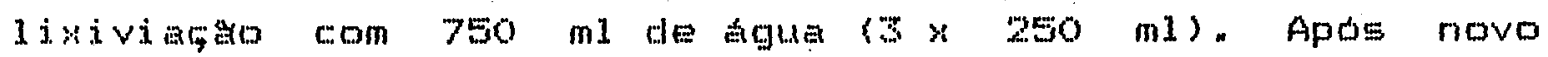
periodo de duas semanas form adicionados mais 700 ml de Agua (7x $100 \mathrm{ml})$ mpos igual periodo mais $700 \mathrm{ml}$ $(7 \times 100 \mathrm{ml})$ 
Ciada coluria recobeu ao todo 4.650 m. de agua,

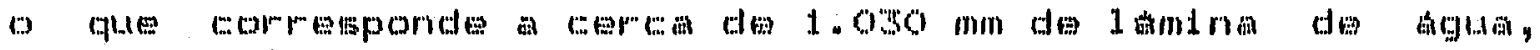
considerando a drea da superficie das colunas.

\section{2 .3 .4$. Coleta des amostras}

Apds os miclos de lisiviagaro os segmentos

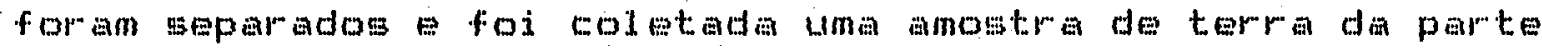

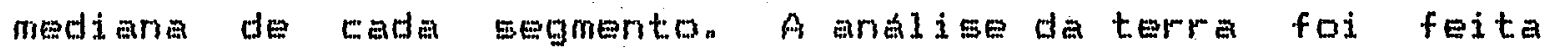
seguindo metodologia descrita no item 3.3 .1 .

\section{3. Metodoloi anditica}

\subsubsection{And 11 世政}

OE Vasos for am amostrados rom coletor de amostras cilindmico, retimandomse g anostras simples em diferentes posictoses de mperficie do Vaso, obtendome dessa maneira uma amostra composta por vaso.

Tomoumse o cuidado de no coletar amostras na posiço en que foi 1 ocalizado o Suporte Capilar.

As amostras de terre for am secas ao ar e

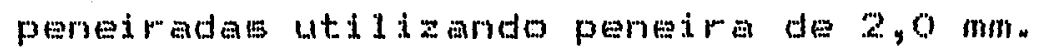


A materia orgunica foi determinada, 3 WALKLY Utilizandowate $1,0 \mathrm{~cm}$ de term, pelo metodo de WALFLEY \& BLACK (1954). Nesse metodo a materi a organica b oxidada por solucato de dicromato de potassio em presenca de fido sul furico. A titulata do exisesso de dicromato fe feita com

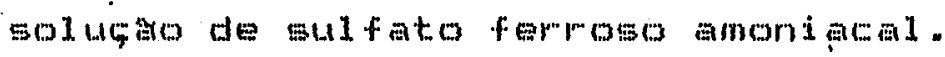

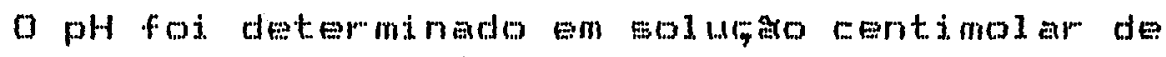

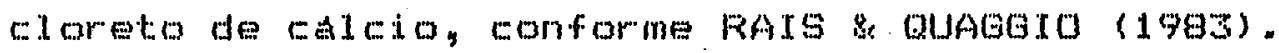

A avaliacho da acidez potencial, isto e, o Valor de $H+A 1$, foi fejta pela depresso causada no pH de una soluço tampaso, conforme deserito em RATS \& QUAGGIO $(1965)$

O fosforo, calcioy magresio potassio for am

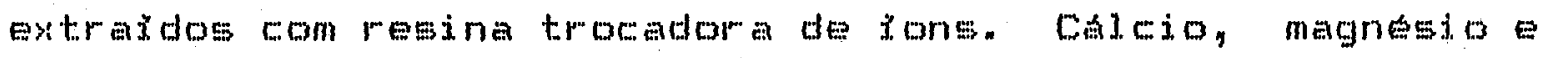
potassio foram determinados por espectofotometria de

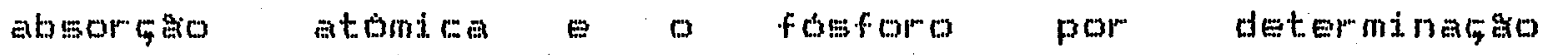
espectrofotometrica da cor azul do compleso fosfomolibdico formado entre o fosfato molibdato, gegundo metodo demsito pOr RAIS Q QUAGGIO (1983).

o ensofre na forma de sulfato do 5010 foi determinado pelo metodo tumbidimetrico conforme VITTI \& SUZUK: (1979). 


\subsubsection{Andides do material vegetal}

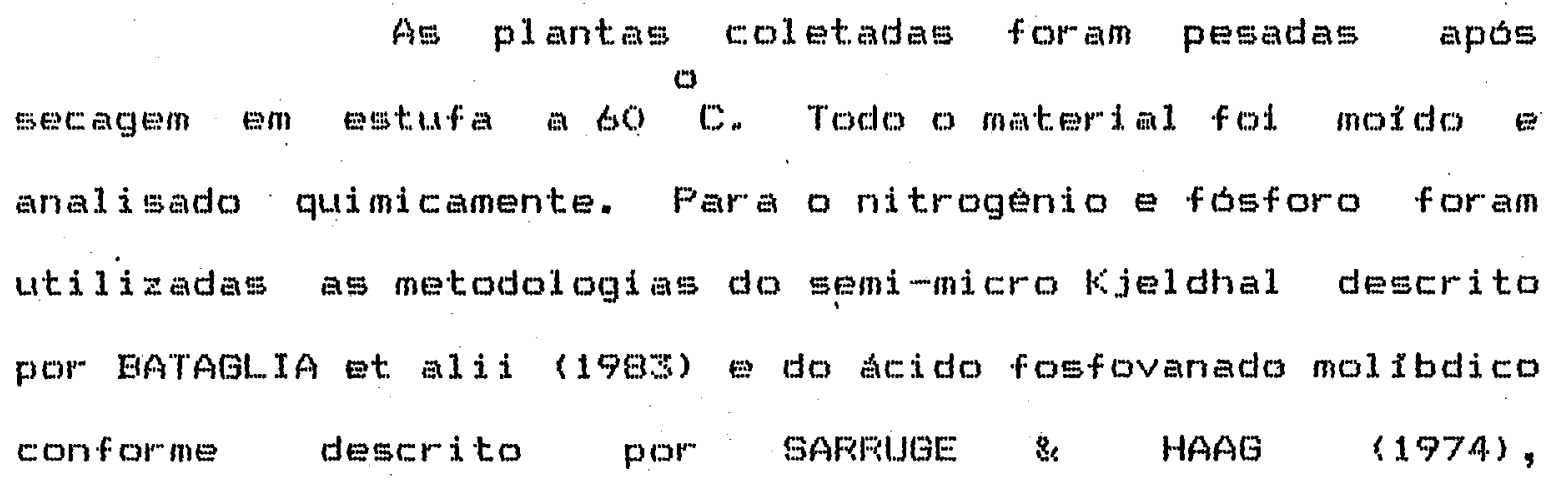
mepectivamente.

Parm a determinacio do potassio, calcio, magnesio micronutrientes utilizoum a mpectrofotometria de absorfaco atomican, conforme descrito por JOFEENGEN $(1977)$ 


\section{RESULTADOS, DISCUSSAO E CONCLUSOES PARCIAIS}

\section{1. Ensaio de efeito pesidual}

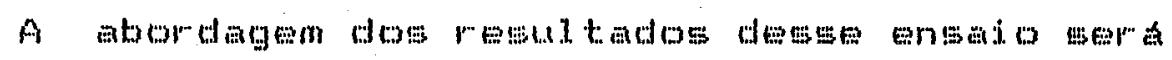
feita da maneira descrita a seguir.

A produca de materia sece por vaso serí utilizada como indicador da eficana dos diferentes tratamentos os outros pametros arialisados pteor de alguns nutrientes na parte aerea das plantas e complexo de troca do solo) servilua para explicar o comportamento das plantas. Fara tanto apumsentaremos inicialmente os valores da producto de materia duplas entre os tratamentos e as interacbs entre as tratamentos o

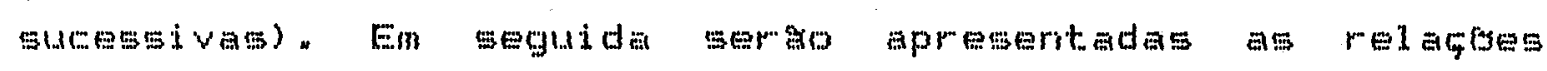
significativas entre os tratamentos e entre os tratamentose as fpocas dos outros parmetros analisadose que podem explicar o comportamento des plantas

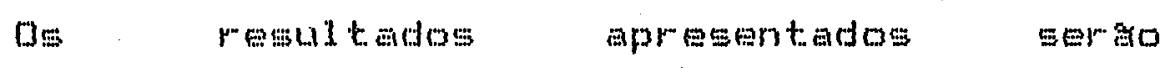


poster iormente discutidose sergotiradas as conclusöes do ensaion

No Quadro 2 (bsan 7072 ) esta as interacoses entre os tratamentos, no oudro 3 (pag. 73 e 74 ) as intermedes entre tratamentos e epocas de corte $a$ no Quadro 1 (paga 69) as medias gerias dos tratamentos. os resultados das andises de terra estäo no Quadro 5 cág. 76 a 78) (interacoses entre tratamentos) a no buaro 4 (pas "75) (medias gerais).

\section{1.1. Resulados da an 1 ise das plantas}

A.1.1.1. Materia seca $(9 / 4 a n o)-M .5$.

a) Interactenentre tratamentos

A maior producto de M. B. ocormen nos tratamentos FS EFS + SC (em media 8,6 g/vaso) e a menor - 7.6 g/yaso - no tratamento sc. com o aumento das doses houve um aumento da producto ate a gltima doses No LR as productes foram quase o dobro das poducose do LF $(6,0$ g/Yaso no LE e 19,6 , g/mo no LR). A Calagem näo teve nenhum

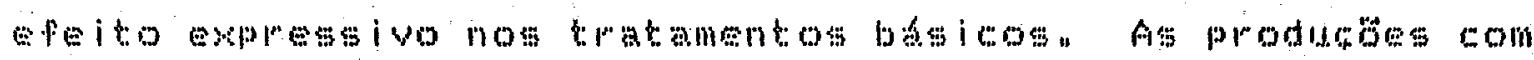
E sem calagem nos tratamentos SC, FS FS + SC foram muito semelhant:es a 


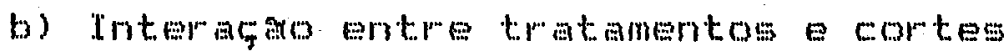

Nos tros tom amentos as produceses decreserem do primeiro ao quarto corte. Apenas no primeiro corte houve diference significativa entre os tratamentos. Nesse corte o tratamento FS + SC apresentou maior produça e o SC a menor.

Novamente ä calagen teve pouca influsneia na

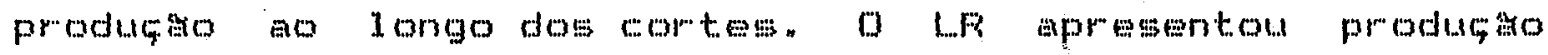
significativamente superior nos quatro cortes.

\subsubsection{Nitrogendo (\% nat folna) $-N$}

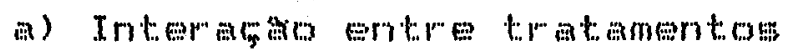

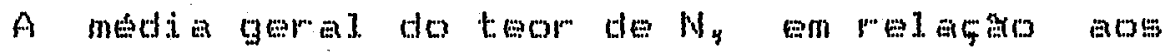
tratamentos basicos, a wa agom e abs eolos pouco variavel

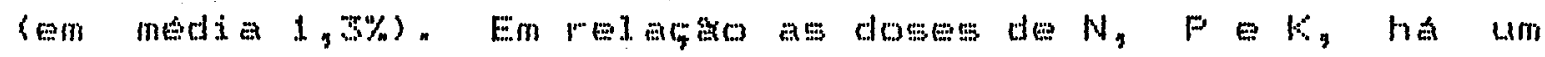
aumento de $1,2 \%$ de doese 0 a $1,6 \%$ na dose 3 .

No LF, em todas as doses os teores de $N$ for am significativamente superiores a

b) Interargarostoretratamentos e comtes

Nos quaro cortes o teor de $N$ foi 
praticamente iqual nos tres tratamentos basicos Ha um

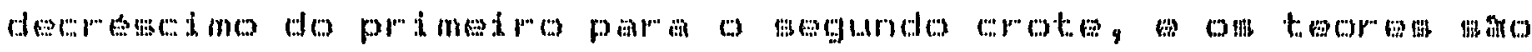
Eemelhantes do segundo a purrto corte.

No primejo corte ha um decresecimo do teor de W da cose tres perm a zero. Do segundo corte en diante, os teores de N nato apresentam diferencas nas quatro doses.

\subsubsection{Fowforo (\% Ma folha) - F}

Dos tress tratamentos bisicos o SC apresentou os menores teores de $F(0,75 \%)$. Os tratamentos FS e FS+ SC, com 0,99\%, apresentaram teores un pouco mais elevadosn

Com a Ia agem trouve uma diminuica do teor de F na folha.

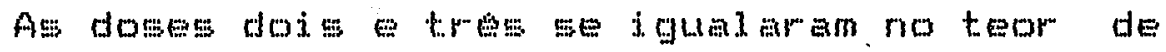
P. Ate a dowe dod houve um amento griativo de Po"

Nos rese tratamentos ha lum aumento do teor de

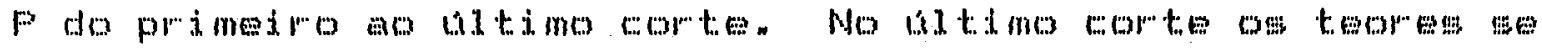
igualam nos tres tratamentos basicos. No primeiro comte o toor mai elevedo foi encontrado no FG $(0,89 \%)$ seguido do $F G+S C(0,75 \%) \equiv 5 C(0,53 \%)$ 
Nos guaro cortes, a calagen diminuiu

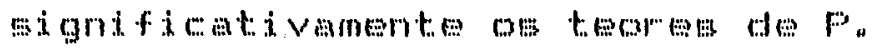

Entre os dois solos nás houve uma tendencia definida dos teores de F a 1 ongo do tempo.

Na primeir sporea as doses dois e tres

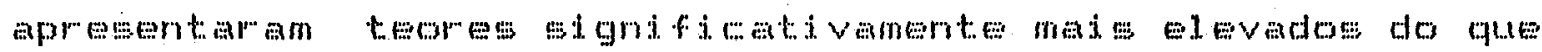
as dum doses inferiores. Com o tempon essas diferencas dimimuir am os teores aument ar" ama

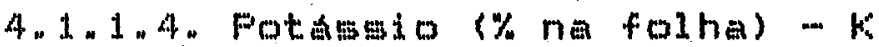

0 teor de

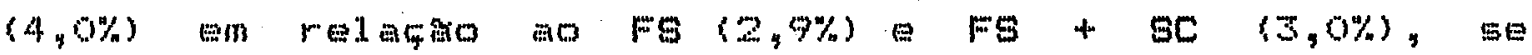

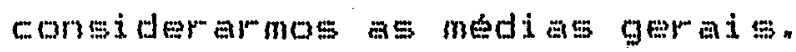

MO LR o teor de foi major ae comparado a LE.

Com o aumento da dose de $M, P$ e ho hama

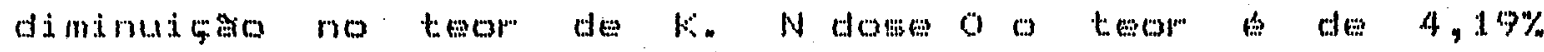
dimimuindo gradativamente atd 2 gr\% ni dose 3 . Entretanto,

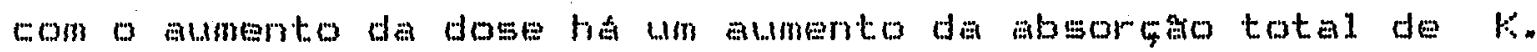
Na dose 0 o total de $k$ absorvido dose $3 \quad 32,5 \mathrm{mg} / \mathrm{V}=0$. 
Nos tres tratementos basicos o LF apresentou

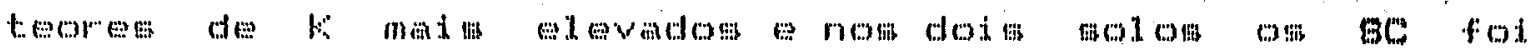
significativamente superior"

Nos tres tomtamentos hé um decrescimo do teor

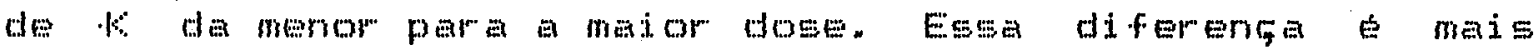
acentuada nos tratamentos FSEFS + SC do que no SC. A

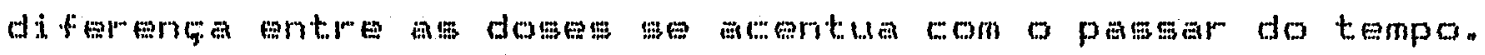

Nos tres traternentos besicos he uma diminuigo do teor de k do primeiro a quaro corte e em todos os cortes o SC apresentou valomes significativamente superiores aos outos dois tretementos.

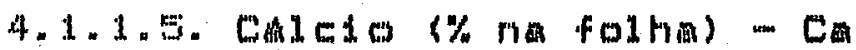

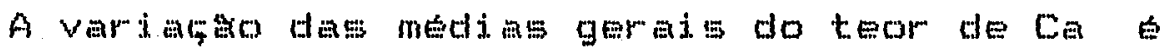
muto pequena As maiores diferencas foram encontradas

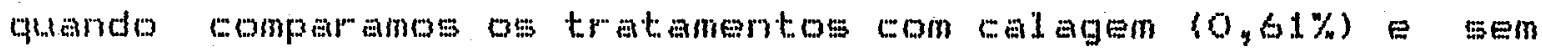

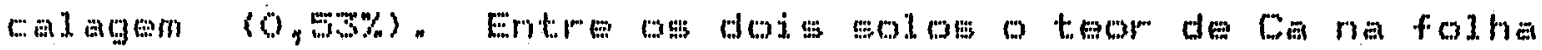
foi maior no LE. Es interacoses solo $x$ tratemertos principais, calagen $x$

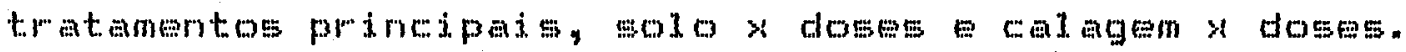

Nos tom tratamentos basiros há um aumento no teor de Ca ao 1 ongo do tempo, motretanto o total de Ca 


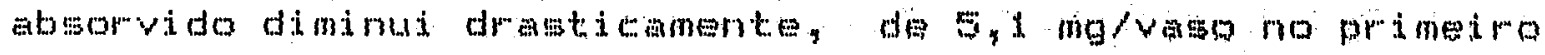
rorte a $2,7 \mathrm{mg}$ -

Os tratamentos con wa agen mantiveram teores

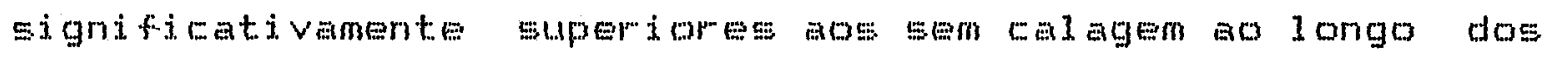
quatio cortes.

\subsubsection{Magnemio (\% na folha) - Mg}

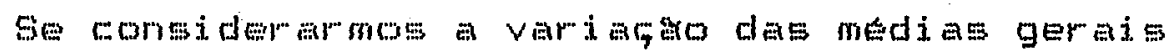
dos tratamentos percebemos que apenas a calagem motivou

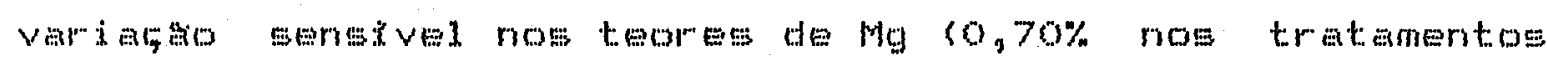
com calmgem e $0,33 \%$ nos tratamentos $\operatorname{sem}$ calagem). No

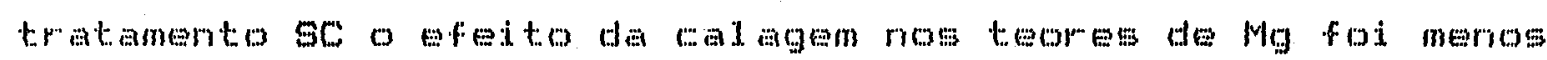
expressivo. No LE a Ealagem amentou os teores de Mg mais do que no L.F.

Ao 1 ongo dos quatro tortios hat um tendencia do aumento do beor de Mg nos tres tratamentos bésicos. Nos

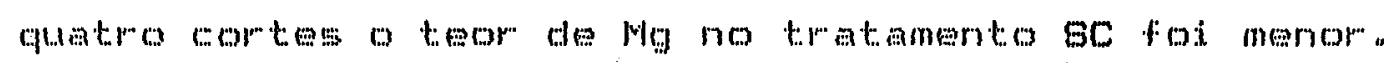

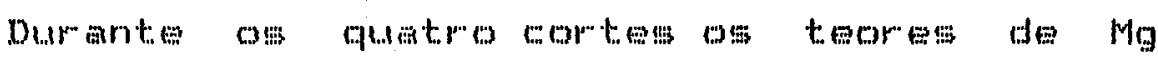
permanew mais elovadom mas foltas das plantas que

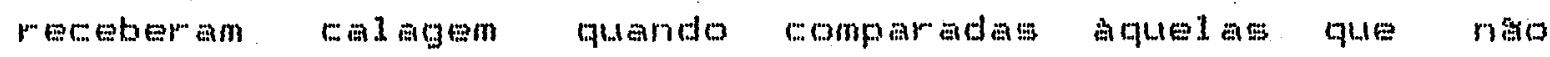
remeberm. Essa diferenca permanece praticamente constante im tom orno of $0,4 \%$. 


\subsubsection{Manganes (ppm na folma) - Mn}

Considerando as medias gerais dos tratamentos

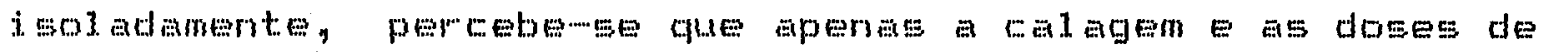
N, F a K afetaran sensivelmente o teor foliar de Mr. Nos

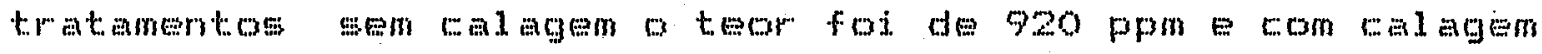
190 ppm. Com o aumento da dose de $N_{y} F$ e ha tamben um aumento do teor de Mr. de 324 ppm na dose o para 799 ppm na $\cos 5 \mathrm{~s}$

Noto ha una tondencia muito bem definida de variaga do Mn ao 10 gog dos quatro cortes en relacas aos tratamentos.

\section{1. 1.8. Zinco (ppm na folha) w $\mathrm{Zn}$}

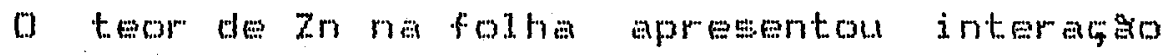

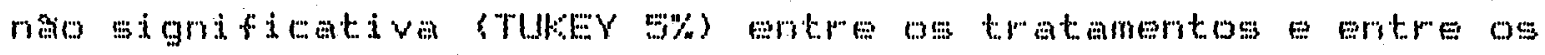

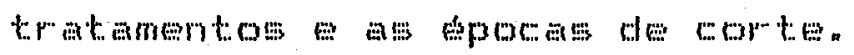

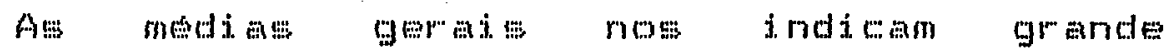
uniformidade dos valores com uma molitude de variaço de 495 ppm na dose o a 551 ppm na dose 2. Os valores dos outros

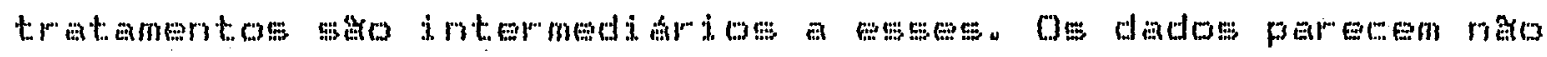
ter sido afetados pelos trat amentos apl it ados. 


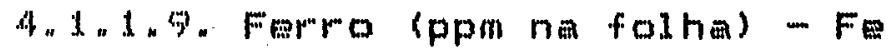

As median gerais now indicam que o teor de Fe

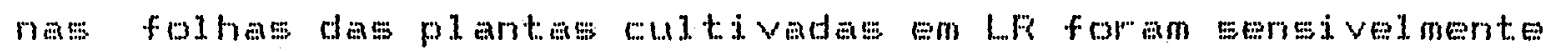

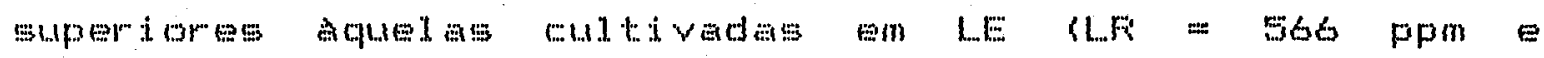
LE

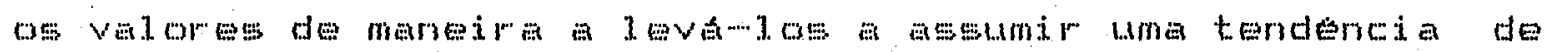

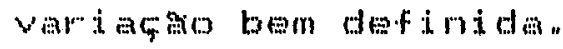

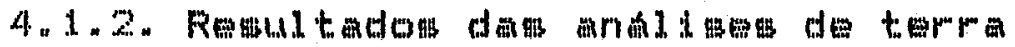

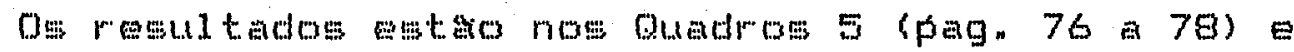
4. (pagr. 75).

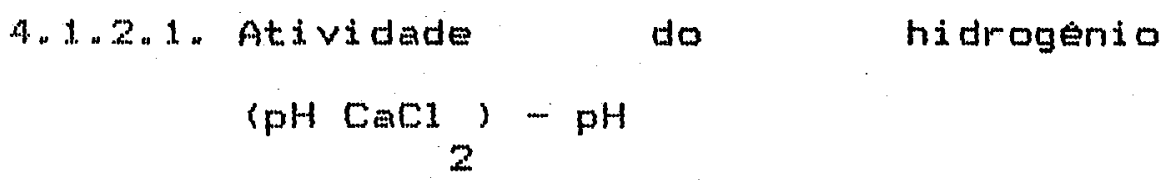

A medi a geral do valor pH dos tratamentos ह muto semelhante, igualmente orom"re entre os solos e doses.

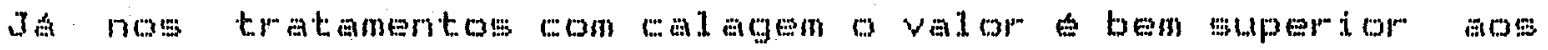

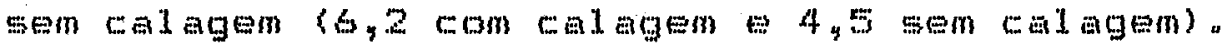

De valom mais baixos de pH nas quatro doses ocorrerm no tratamento FS.

A wal agen elevou mate o val or" ph no LE do que 


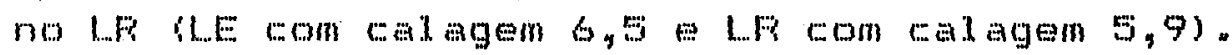

\subsubsection{Fonform soluvel \{ug/em $\}^{3}$ ) $-F$}

Amalisando as medi as gerais dos tratamentos observase que o teor mais baxio de F ocorreu no tratemento 3 .

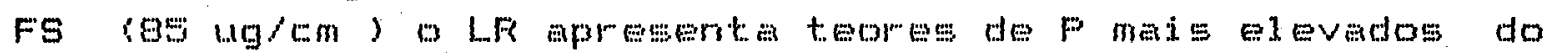

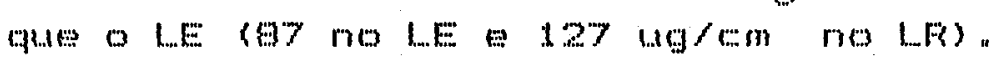

Com o aumento da dose de $N, F$ e $k$ há lum aumento equivalente do teor de p no moloy paseando de um teor baiso a elevado ( 12 na dose 0 a 246 ug/cm na dose 3 ).

Nas primeir as tres doeses os valomes de P. So

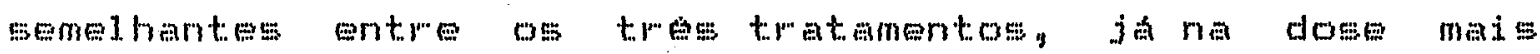

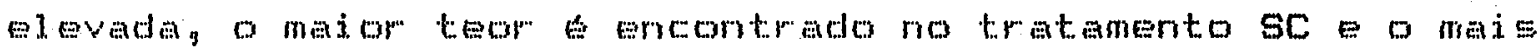
baixo Mo FS.

No LF com excerso da dowe o, em todas as outras, o toor de Pe mat el Evado do que no LE.

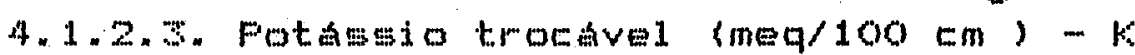

As medis gerais do tew dek no solo wo multo semeitiantes. 
Na inter acoso entre tratimentos, apesar de

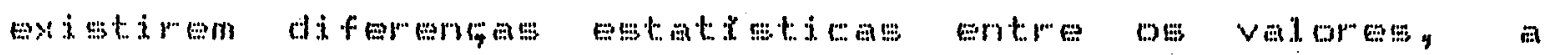
variaga nominal e muto pequena nato sendo poservel

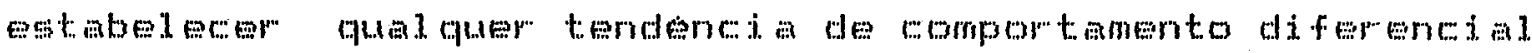
em rel acto aos tratamentom.

\subsubsection{Colcio trocavel (meq/100 $\left.\mathrm{cm}^{3}\right)-$ -}

Analisando as medias geris dos tratamentos observame que os teores de Ca mais elevados estaro associ ados aOs tratamentos com adica de SCs (SC E FS + SC).

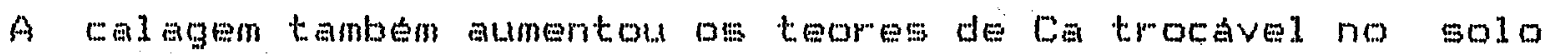

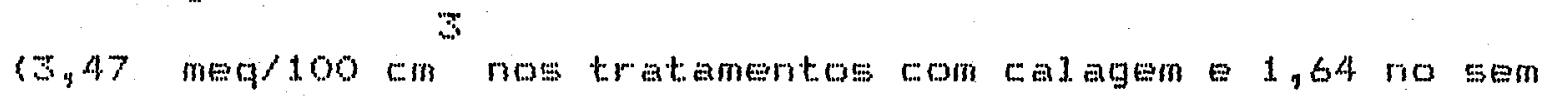
Ealagen, en media).

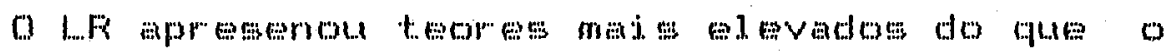
$\exists$

LE (1,92 nO LE E 3,19 meq/100 (in nO LF).

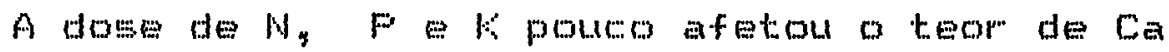
nos $=010$.

No tratamento Feg no houve diferenga do teor de Ca om relaço as doses ja nos outros dois tratamentos ha una tendencia de aumento do teor de Co com o aunento da dose $d e N, F \notin K_{4}$ 


\subsubsection{Magnomio trok avel (meq/100 em $1-M$}

Analismoso as medias gerais dos tratamentos,

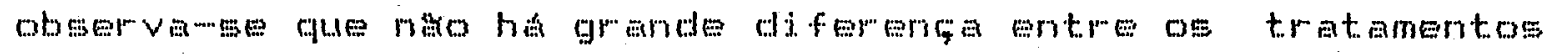

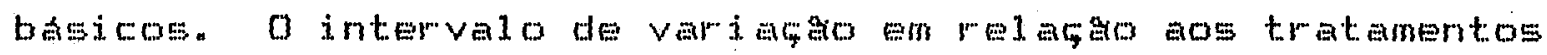

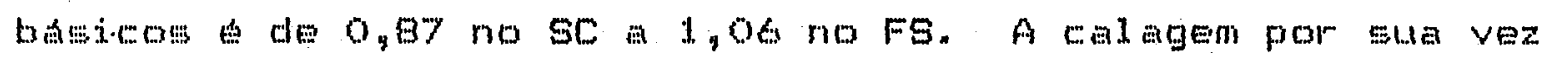
aumentou comei der avel ments o teor de Mg. Nos tratamentos sam

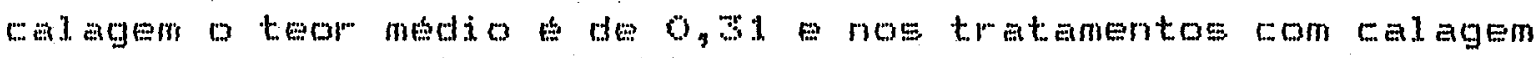

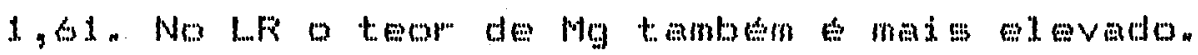

Entre as difermontes doses de Ny P e nas há grande variaca no teor de $M g$.

\subsubsection{Goma de bases (meq/100 $\left.\mathrm{cm}^{3}\right)-5$}

Analisandome a media geral dos tratamentos observame que a $S$ e 1 igeiramente muperior nos tratumentos SC F F + SC 3,99 3,77, rospectivamente. DS tratamentos

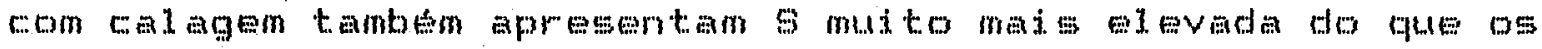

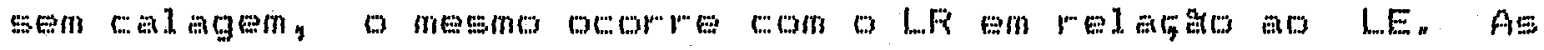

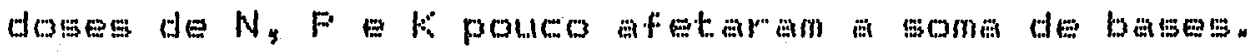
4.1.2.7. Capacidade de troca de cations

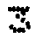 (meq/100 $\mathrm{cm})$... CTC

Analisandowe a modia gemal dos tratamentos, 
observane que ha poumo oifererca entre a CTC dos

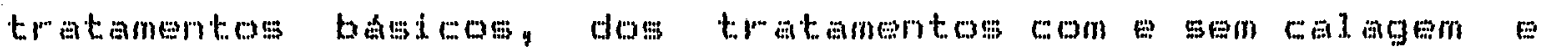
entre as diferentes doses. Aperias a CTC do LF E meior do que a do LE, $4,44 \in 9,17$, respectivamente.

\section{1.2.8. Saturario por bases $(\%)-v \%$}

Felas morias gerais observas que dos tratamentom isoladamente, mpenas a ralagem afetou a variaca da saturafo por bases em mism proporcaso $474 \%$ nos tratamentos com cal agm $31 \%$ no semy em media).

Nos tratamentos com esem colagem no ho há grande diferencia entre os brese tratumentos basicos.

Nas quatro doses, o kratamento FS apresentou

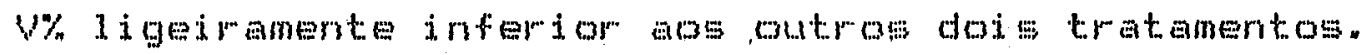

$$
\begin{aligned}
& \text { 4.1.2.9. Ensofm soluvel } \\
& -2 \\
& \text { (s0 } m g / 100 \mathrm{~cm})-50 \\
& 44
\end{aligned}
$$

As medi as gerais nos mostran que nos dois tratamentos com adicäo de SC (SC F maior do que no FG: SC $=99$, FS+ SC=95EFG $=55$. 


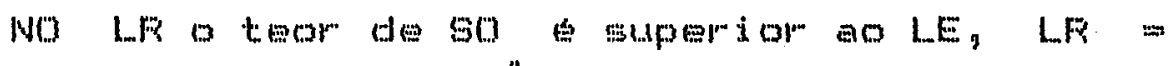
$109-1 E=57$.

Nesses doin solos o toor maje elevado foi observado no tratamento sc.

\subsubsection{Discussơ dos mesultados}

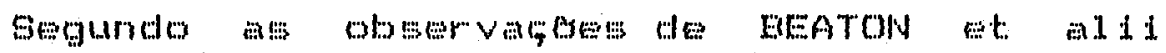
(1967) EOONDUANG et aid (1976) og fertilizantes de solubilizaço lenta, especialmente os nitrogenados, levam as

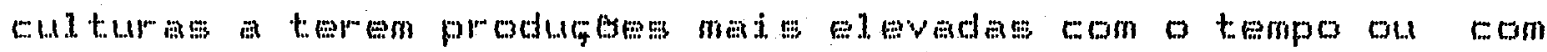
cortes sucessvos quando comparados a formas mais soluveis. A produca inicial das fomas mais soluveis d maior. Desea

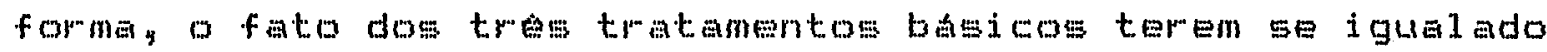
en termos de producto de Materia Gecia a partir do esegurdo conte; indicanos que o $5 \mathrm{C}$ rivo 5 comporta como um

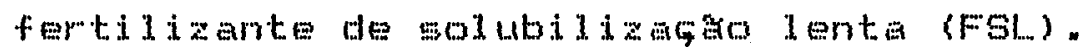

O tratamento Fo + BC apresentou no primeiro corte a mator produça de Materia Seca, seguido do tratemento Fs e por altimo o SC. Fodemos inferir dax, que ha um efeito benefico comendo pela presenta do sc sen fertilizantes, fofeito esso, amulawo quando os fortilizantes sabo intiodus idos no 98. 
A conmtanki do teor de Nitrogenio na folha

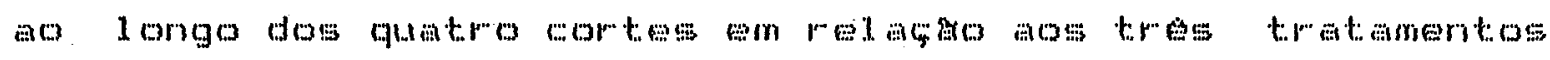

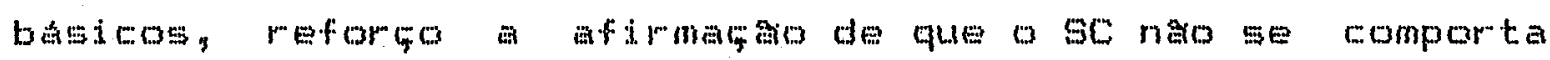

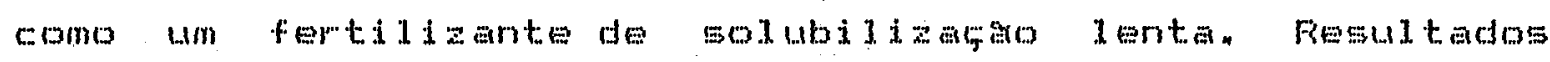
semelhantes form obtidos por VAUSHN bt ali

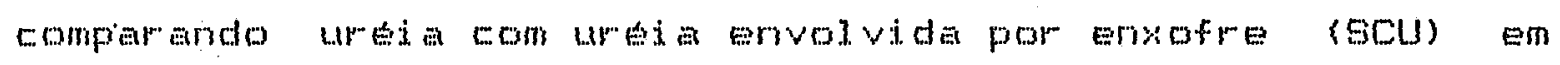

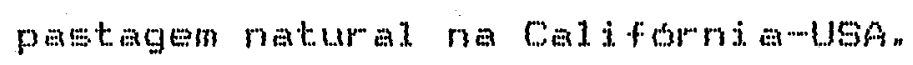

o teor mais elovado de k na folta do tritamento se ao longo dos puatro cortes, nos dois solos, com esem calagerm nas quatro doses de N, Pe ka indica que ha um aproveitamento melhor do k quando apliaado dessa

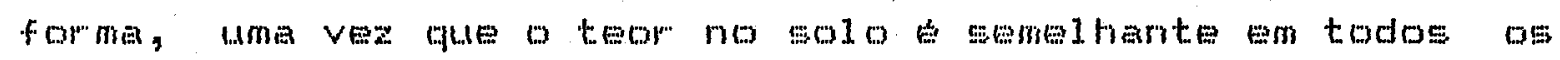
tratamentos. Este fato pode ser explicado devido a lma menor lixiviarga do k aplinado desen forma

Em relario aso tratementos principeds os

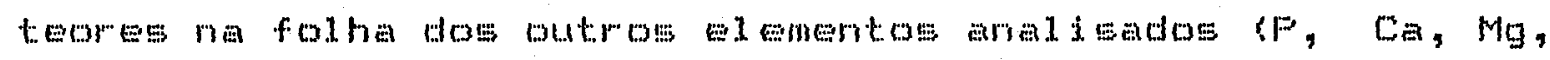
Mny Fe $F$ an apresentaram variacoss ou interacoes significativas, mas no soguindo uma tondencia gue expli gue

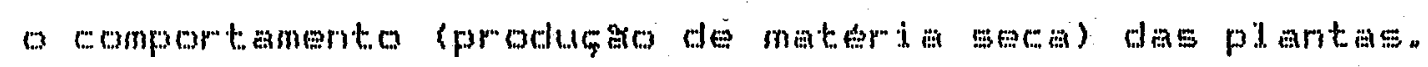

A peguena variara do $N$ nos tratamentos

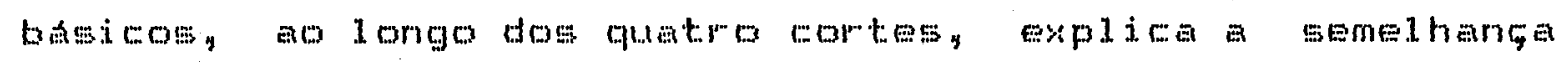
das producbes de materia seca. O teor maj elevado de b na folne, no tratamento SC Em todas as mas interacoes, 1eva- 
nos: a inferir uma melhor absorbo de $k$ quando este e aplicaco vin $5 C_{0}$

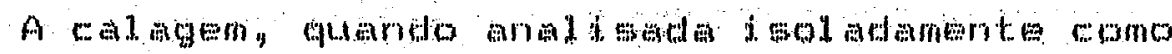
tratamento, provocou un mumbo da producro demateria seca (M. . .) porem esse aumento foi multo inferior aquele devido ao efeito isolado dos solus. fom a calagen observams teores

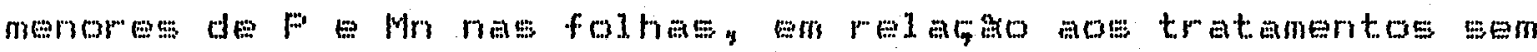
calagem. O feito da ciagen desaparece a partir do terreiro rorte, igual andome a partir dia a produg do de M.S..

A media de producta no LF de 10,6 g/vaso e no LE 6,0 g/vaso. No LF observa-ge teores ne for

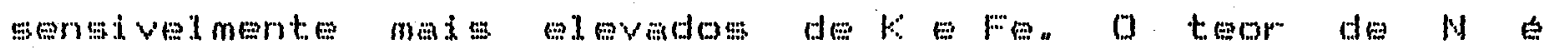
semelhante. A producto nos dois solos diminui do primeiro ao

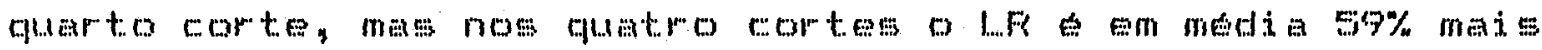
proututivo.

Observarsen analisando as doses isoladamente, que hat Lin aumento de producto de M. 5. da menor para a maior doce. Da dose o para a 10 enmento e de $64 \%$ da 1 para a 2 , $79 \%$ da 2 paria

EEs asumento vom acompanhedo de um aumento do teor de $N$ na follia de $1,2 \%$ na dose 0 para $1,6 \%$ na dose $Z$. 0

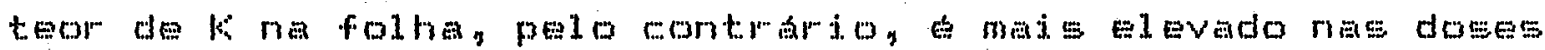


menormes.

As difersencas de produrgo entre as doses se mantem durante os quatim cortese apesar de haver uma diminuica do primeino ao quarto corte em todas as doses. o teor de N na folba abmenta da dose o para a 3 apenas no primeiro corten nos tres cortes subsequentes os valores no

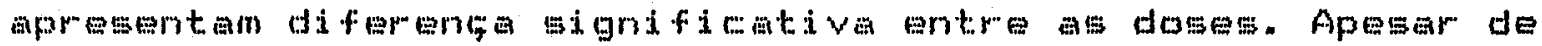

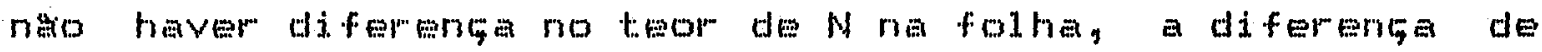
producto de Materia sem existiun

Quprincipais efejtos dos tratamentos basicos nas propriedades do wolo, relacionamere aos teores de Py Ca, V\% $=5-50$ nosolo. O valor pH osteores de for am sensivelmente alterados Ese efeito e devido principalmente pela presenga do sc e nao pela colocaca ou noto fos fertiliantes an seu interior, uma vez que os tratamentos SC FG o teor de P solivel \& de 127 ug/en no tratamento $9 \mathrm{C}, 109$ no FS + SC $=85$ no FG.

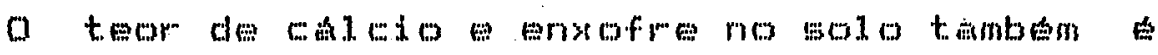

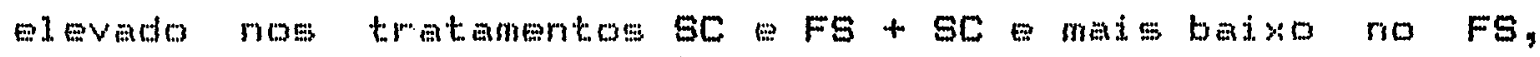

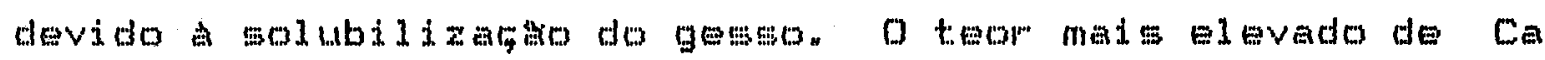

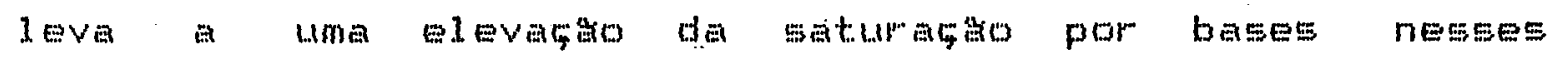
tratamentos. 


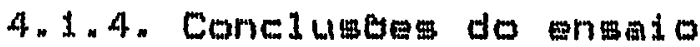

a) $0 \mathrm{sc}$ naco se comporta como um Fertilizante

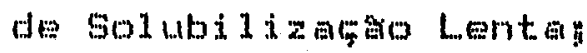

b) O K quardo aplitado via SC E melhor aproveitacto $p=1$ as $p l$ antas

c) o efeito do tipo de solo na produgas analisado isoladamente maior do que o efeito da calagem e

d) os tratamontos basicos nao afetiram o Valor pH, e os teomes de Ki Mg no solo. As Vari aços de F, Cia e 5-50, encontratas no 4

tratamentos, devem-se principalmente a presenga do SC. 


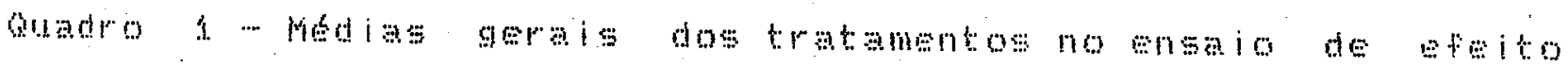

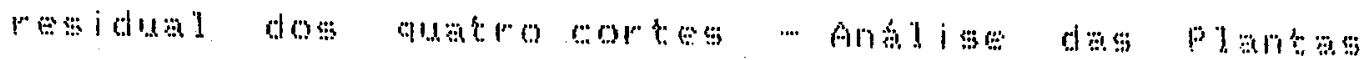

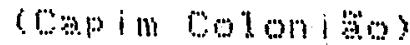

PARABETRO

\begin{tabular}{|c|c|c|c|c|c|c|c|c|}
\hline$M .5$. & $N$ & $P$ & $k$ & $\mathrm{Ca}^{\prime}$ & $\mathrm{Mig}$ & th & $\mathrm{Zn}$ & $\mathrm{FE}$ \\
\hline$g$ & 7 & $\%$ & 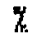 & $\%$ & $\%$ & Ppll & ppm & $\mathrm{prg}$ \\
\hline
\end{tabular}

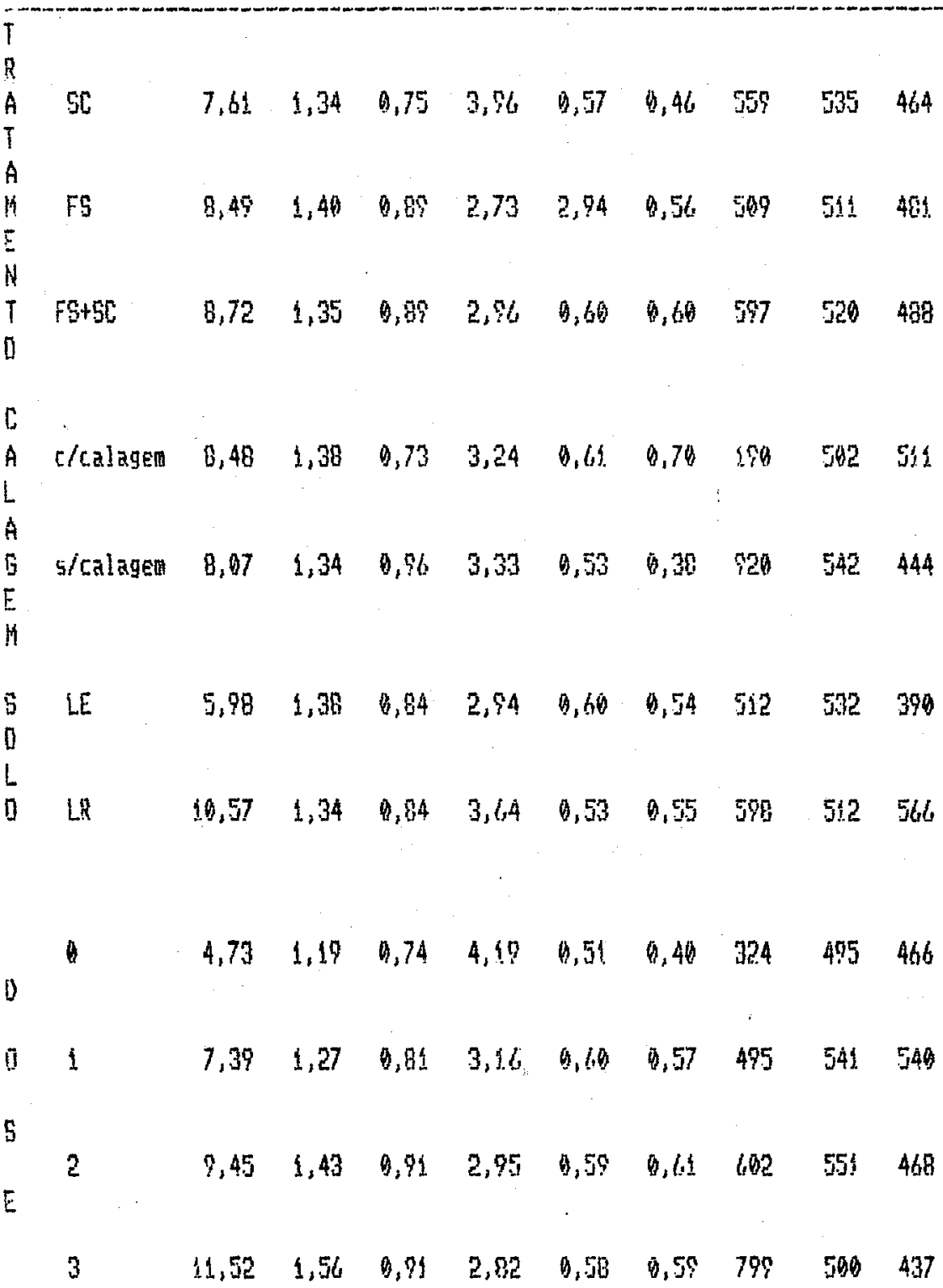




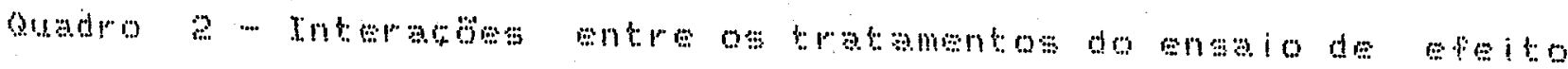

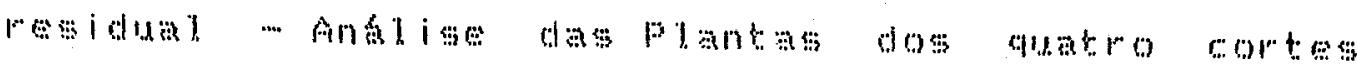
(mopim oloniso)

\begin{tabular}{|c|c|c|c|c|c|c|c|c|c|}
\hline & \multirow{2}{*}{ TRATAMENTO } & \multicolumn{2}{|c|}{$50<0$} & \multicolumn{2}{|c|}{$D A L A E Y$} & \multicolumn{4}{|c|}{$D O S E$} \\
\hline & & LE & LR & roth & SEH & $\theta$ & 1 & 2 & 3 \\
\hline 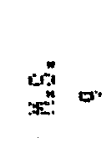 & $\begin{array}{c}S C \\
F S \\
F S+C C\end{array}$ & & & $\begin{array}{l}7,55 \mathrm{Ab} \\
8,96 \mathrm{Az} \\
6,99 \mathrm{Az}\end{array}$ & 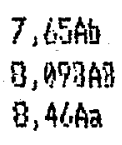 & $\begin{array}{l}4,680 a \\
4,700 a \\
4,730 a\end{array}$ & $\begin{array}{l}6,34 C b \\
7,44 \mathrm{Ca} \\
8,21 \mathrm{Ca}\end{array}$ & $\begin{array}{r}7,64 \mathrm{Bc} \\
10,30 \mathrm{Ag} \\
14,42 \mathrm{Az}\end{array}$ & $\begin{array}{l}11,7 \mathrm{EAa} \\
11,25 \mathrm{Aa} \\
11,55 \mathrm{Ba}\end{array}$ \\
\hline 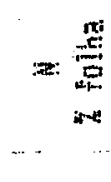 & $\begin{array}{c}5 c \\
F 5 \\
F 5+5 C\end{array}$ & $\begin{array}{l}1,33 \mathrm{Ab} \\
1,44 \mathrm{AB} \\
1,40 \mathrm{AB}\end{array}$ & $\begin{array}{l}1,35 \mathrm{Ad} \\
1,37 \mathrm{Ba} \\
1,303 \mathrm{~b}\end{array}$ & & & & & & \\
\hline a. $\underset{n}{\stackrel{n}{E}}$ & $\begin{array}{c}5 C \\
F S \\
F S+5 C\end{array}$ & $\begin{array}{l}0,67 \mathrm{Bb} \\
0,94 \mathrm{~A} \\
0,92 \mathrm{Aa}\end{array}$ & $\begin{array}{l}0,83 \mathrm{Az} \\
0,8 \mathrm{BAa} \\
0,8 \angle \mathrm{Az}\end{array}$ & & & $\begin{array}{l}0,75 \mathrm{Aa} \\
0,7 \mathrm{CA} \\
0,76 \mathrm{Ba}\end{array}$ & $\begin{array}{l}0,74 \mathrm{Ab} \\
0,940 \mathrm{a} \\
0,85 \mathrm{ara}\end{array}$ & $\begin{array}{l}0,74 \mathrm{Ab} \\
1,63 \mathrm{Aa} \\
9,97 \mathrm{Aa}\end{array}$ & $\begin{array}{l}0,78 \mathrm{et} \\
0,97 \mathrm{ad} \\
0,99 \mathrm{Aa}\end{array}$ \\
\hline$w \frac{0}{\infty}$ & $\begin{array}{c}S C \\
F S \\
F S+5 C\end{array}$ & $\begin{array}{l}3,71 B \mathrm{z} \\
2,55 \mathrm{Bb} \\
2,56 \mathrm{Bb}\end{array}$ & $\begin{array}{r}4,21 A \\
3,33 A B \\
3,37 \text { AD }\end{array}$ & $\begin{array}{l}4,06 \mathrm{Ad} \\
2,778 \mathrm{~b} \\
2,71 \mathrm{Ab}\end{array}$ & $\begin{array}{l}3,35 \mathrm{Z} \\
3,11 \mathrm{Ab} \\
3,62 \mathrm{Ab}\end{array}$ & $\begin{array}{l}4,22 \mathrm{AB} \\
4,23 \mathrm{Aa} \\
4,14 \mathrm{Aa}\end{array}$ & $\begin{array}{l}3,71 \mathrm{~A} \overline{\mathrm{a}} \\
2,7 \mathrm{Bb \textrm {b }} \\
2,83 \mathrm{bb}\end{array}$ & $\begin{array}{r}3,6933 \\
2,5486 \\
2,3586\end{array}$ & $\begin{array}{l}4,92 \mathrm{Az} \\
2,23 \mathrm{Ch} \\
2,24 \mathrm{Cb}\end{array}$ \\
\hline 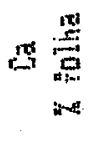 & $\begin{array}{c}55 \\
F 5 \\
F 5+5 C\end{array}$ & $\begin{array}{l}0,41 \mathrm{Aa} \\
0,37 \mathrm{Ab} \\
0,43 \mathrm{Aa}\end{array}$ & $\begin{array}{l}0,350 \mathrm{~b} \\
0,75 \mathrm{Ab} \\
0,388 \mathrm{a}\end{array}$ & $\begin{array}{l}0,62 \mathrm{Aa} \\
0,50 \mathrm{Ab} \\
0,63 \mathrm{Aa}\end{array}$ & $\begin{array}{l}0,5286 \\
0,5676 \\
0,578 a\end{array}$ & $\begin{array}{l}0,550 \mathrm{a} \\
0,4500 \\
0,539 \mathrm{a}\end{array}$ & $\begin{array}{l}9,59 \mathrm{Ab} \\
0,57 \mathrm{Ab} \\
0,63 \mathrm{Aa}\end{array}$ & $\begin{array}{l}0,59 \mathrm{Aab} \\
9,5 \mathrm{NAS} \\
0,62 \mathrm{Aa}\end{array}$ & $\begin{array}{l}0.54 \mathrm{Bh} \\
0.57 \mathrm{Ab} \\
0.63 \mathrm{~A}\end{array}$ \\
\hline 몬 & $\begin{array}{c}5 C \\
F 5 \\
F 5+5 C\end{array}$ & & & $\begin{array}{l}0,5 B \mathrm{AC} \\
0,75 \mathrm{Ab} \\
0,7 \mathrm{AA}\end{array}$ & $\begin{array}{l}0,341 \mathrm{c} \\
0,3786 \\
0,423 \mathrm{a}\end{array}$ & $\begin{array}{l}0,417 \mathrm{a} \\
0,368 \mathrm{~b} \\
0,42 \mathrm{a}\end{array}$ & $\begin{array}{l}0,40 \mathrm{AB} \\
0,64 \mathrm{Aa} \\
0,64 \mathrm{Aa}\end{array}$ & $\begin{array}{l}0,52 \mathrm{Ab} \\
0,63 \mathrm{Aa} \\
0,67 \mathrm{Az}\end{array}$ & $\begin{array}{l}0,439 \mathrm{~b} \\
0,65 \mathrm{AB} \\
0,6 \mathrm{AAz}\end{array}$ \\
\hline 돌 & $\begin{array}{c}5 C \\
F 5 \\
F 5+56\end{array}$ & & & $\begin{array}{l}1818 \mathrm{a} \\
294 \mathrm{~B} a \\
195 \mathrm{ba}\end{array}$ & $\begin{array}{l}\text { PBAa } \\
825 A D \\
999 A a\end{array}$ & $\begin{array}{l}334 \mathrm{Ca} \\
390 \mathrm{Ca} \\
3390 \mathrm{a}\end{array}$ & $\begin{array}{l}475 \mathrm{Ca} \\
4820 \mathrm{a} \\
520 \mathrm{Ca}\end{array}$ & $\begin{array}{l}5508 b \\
55696 \\
7908 \mathrm{a}\end{array}$ & $\begin{array}{l}678 A \\
709 A B \\
322 A=\end{array}$ \\
\hline 点. & $\begin{array}{r}\mathrm{SC} \\
\mathrm{FS} \\
\mathrm{FS}+\mathrm{SC}\end{array}$ & & & & & & & & \\
\hline
\end{tabular}

1- Ds espacos en branco referent-se a interabues nẵo significativas

2 - As letras mailsculas diferentes indican diferencas significativas na horizontal (TUREY 5\%)

3 - As letras dinfsculas dierentes indican diferencas signiricativas na vertical (TUKEY 5\%) 
con! inหaเลัด

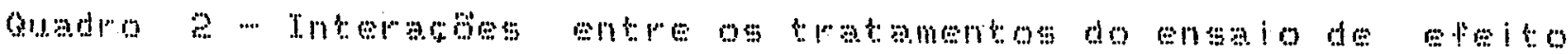

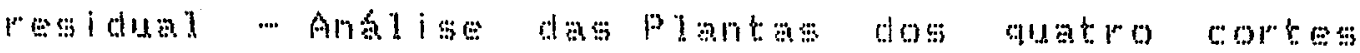
(orm in oloniato)
CALAGEM
D) $5 \mathrm{E}$

SILL

\begin{tabular}{|c|c|c|c|c|c|c|c|}
\hline & & COH & SEH & 0 & 1 & 2 & 3 \\
\hline or & $\begin{array}{l}\text { LE } \\
\text { LR }\end{array}$ & & & $\begin{array}{l}2,360 \mathrm{~b} \\
6,330 \mathrm{a}\end{array}$ & $\begin{array}{l}5,36 \mathrm{Cb} \\
7,4 \mathrm{Cla}\end{array}$ & $\begin{array}{r}7,23 \| 6 \\
11,671 \mathrm{a}\end{array}$ & $\begin{array}{r}8,794 \mathrm{~b} \\
14,84 \mathrm{~A}\end{array}$ \\
\hline$=: \frac{\mathbb{w}}{E}$ & $\begin{array}{l}L E \\
L R\end{array}$ & $\begin{array}{l}1,42 \mathrm{Aa} \\
1,35 \mathrm{Ab}\end{array}$ & $\begin{array}{l}1,35 \mathrm{~A} \\
1,33 \mathrm{~A}\end{array}$ & $\begin{array}{l}1,200 \mathrm{a} \\
1,200 \mathrm{a}\end{array}$ & $\begin{array}{l}1,39 \mathrm{C} \\
1,25 \mathrm{Ca}\end{array}$ & $\begin{array}{l}1,46 B \mathrm{a} \\
1,39 \mathrm{Bb}\end{array}$ & $\begin{array}{l}3,66 \mathrm{Aa} \\
1,52 A \mathrm{~A}\end{array}$ \\
\hline ‥ & $\frac{L E}{L R}$ & & & $\begin{array}{l}0,648 \mathrm{~b} \\
0,354 \mathrm{a}\end{array}$ & $\begin{array}{l}0,78 \mathrm{Az} \\
0,114 \mathrm{Az}\end{array}$ & $\begin{array}{l}0,97 \mathrm{Az} \\
6,85 \mathrm{AL}\end{array}$ & $\begin{array}{l}0,99 A \mathrm{a} \\
0,84 \mathrm{~A}\end{array}$ \\
\hline$\propto \underset{s}{\stackrel{r}{g}}$ & $\begin{array}{l}\text { LE } \\
L R\end{array}$ & & & & & & \\
\hline
\end{tabular}

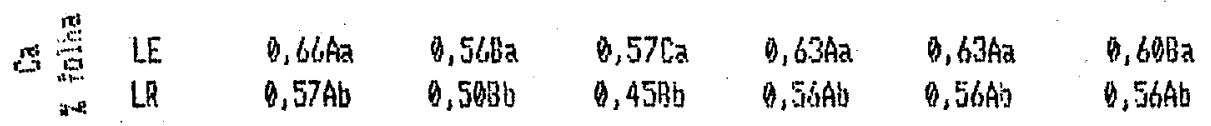

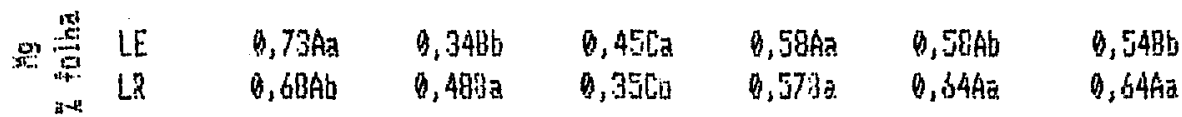

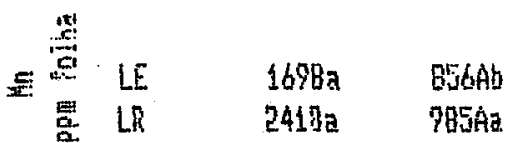

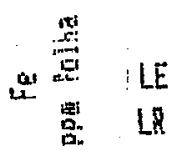

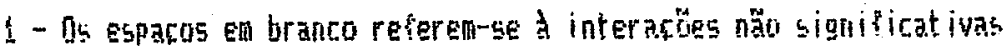

2 - As letras mailsculas diferentes indican diferencas significativas na hor izontal (TUKEY s\%)

3- As letras mindsculas diferentes indican diferencas significativas na. yertical (TUKEY $5 \%$ ) 
cont inและดัด

oudro 2 Interacos antre os tratamentos do ensato de eleito residul … Analse das plantas dos quatro cortes (com im olonios)

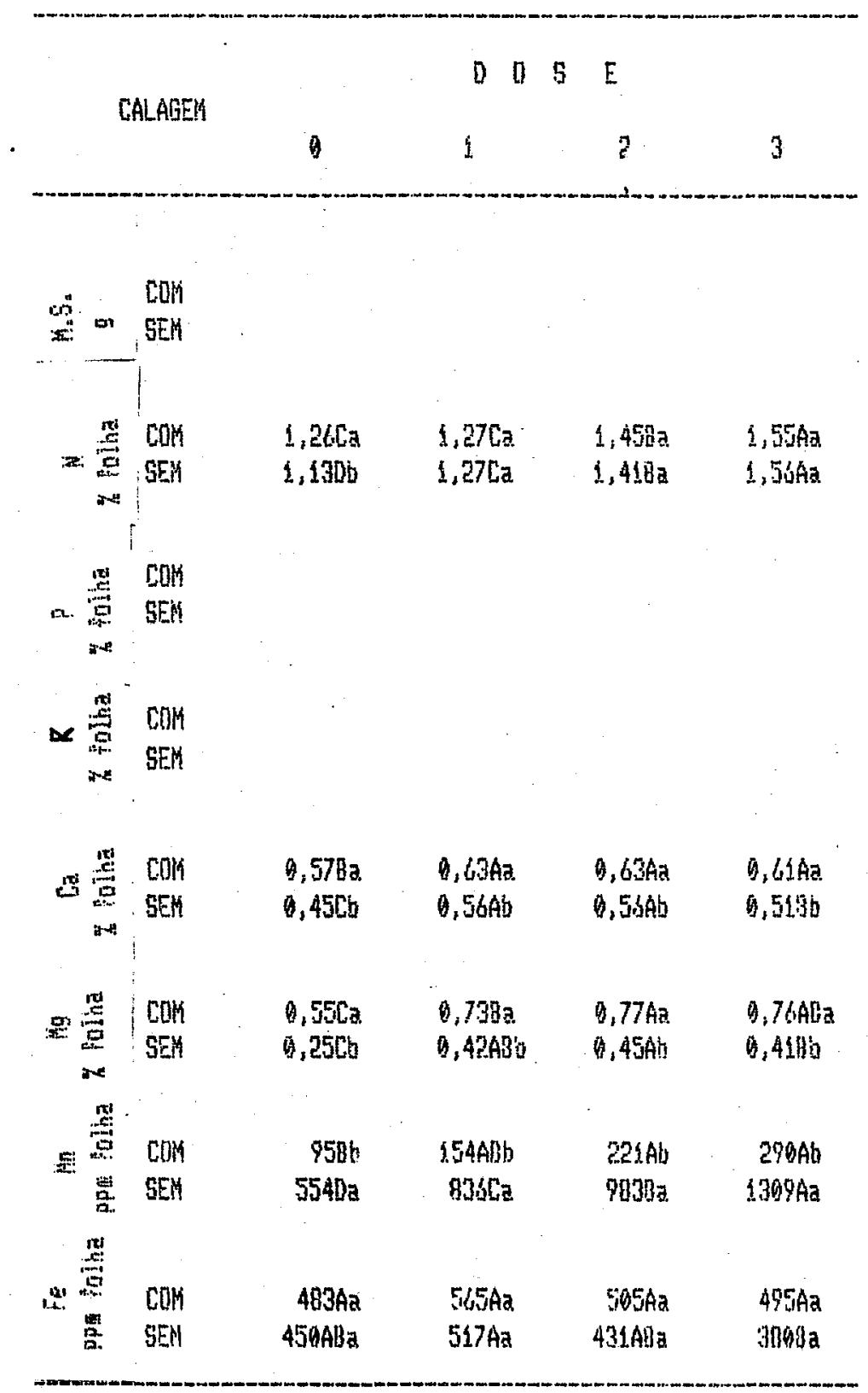

1- Os espacos eq branco refereq-5e à inter acões näo significativas

2 - As letras mailsculas diferentes indican diferencas signilicativas ma horizontal (TUKEY 5X)

3-As letras mindsculas diferentes indican diferencas significativas na. vertical (TUREY $5 \%$ 


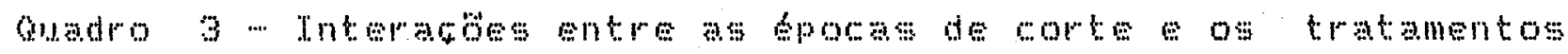

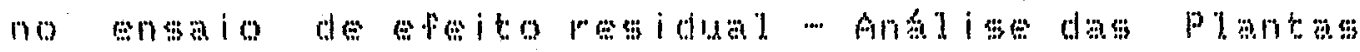
(Capim coloniso)

TRATAKENTOS

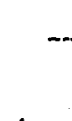

\begin{tabular}{|c|c|c|c|c|c|c|}
\hline \multirow{4}{*}{ 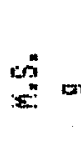 } & 1 & $13,41 \mathrm{Ca}$ & $15,259 \mathrm{a}$ & $16.27 \mathrm{~A}$ & $14,69 \mathrm{JAa}$ & $15,3 L \mathrm{AB}$ \\
\hline & 2 & $9,2 \mathrm{abb}$ & $9,74 \mathrm{Ab}$ & 10,15Ab & 20,89 Ab & $8,76 \mathrm{Bb}$ \\
\hline & 3 & $4,29 \mathrm{AC}$ & $4,93 \mathrm{AC}$ & 4, 6.tARc. & $4,37 \mathrm{AC}$ & $4,37 \mathrm{Ar}$ \\
\hline & 4 & $3,45 \mathrm{Ad}$ & $4,05 \mathrm{Ad}$ & 3. BiAc. & $3,70 \mathrm{Ad}$ & $3,84 A C$ \\
\hline
\end{tabular}
(1)

\begin{tabular}{|c|c|c|c|c|c|c|}
\hline & 1 & 2,13A & $2,22 \mathrm{~A} \mathrm{a}$ & $2,67 \mathrm{Aa}$ & $2,26 \mathrm{Az}$ & $2,01 H_{2}$ \\
\hline & 2 & 1. $13 \mathrm{AC}$ & $1, j B A C$ & $1,6 \angle A C$ & $1.07 \mathrm{AC}$ & $1, B \angle A C$ \\
\hline$=4$ & 3 & $0,96 \mathrm{Ar}$ & $1,0 B A C$ & 1,67 Abr & $\{, B \backslash A C$ & $1,96 \mathrm{AC}$ \\
\hline & 4 & $\{, 25 \mathrm{Ab}$ & $1,21 \mathrm{Ab}$ & $\{, 17 \mathrm{Ab}$ & $1,1 B A b$ & $1,23 \mathrm{Ab}$ \\
\hline
\end{tabular}

\begin{tabular}{|c|c|c|c|c|c|c|}
\hline \multirow{4}{*}{ ㄷ. } & 1 & 0,5350 & Q. BAAD & 0,7580 & $0,61 \mathrm{Br}$ & $0,8 \mathrm{AAC}$ \\
\hline & 2 & $0,558 \mathrm{c}$ & $0,6 \mathrm{BABC}$ & $D, 7 \angle A C$ & $0,598 \mathrm{C}$ & $0,7 \mathrm{GAd}$ \\
\hline & 3 & 0, Q & 0,87 ah & $0,93 \mathrm{Ab}$ & $0,72 \mathrm{Pl}$ & $1,94 \mathrm{AJ}$ \\
\hline & 4 & $1,058 \mathrm{z}$ & $1,13 \mathrm{ABa}$ & $1, j 4 \mathrm{Aa}$ & $1,018 \mathrm{z}$ & $1,20 \mathrm{Aa}$ \\
\hline \multirow{4}{*}{ 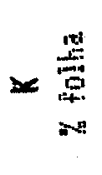 } & 1 & $4,72 \mathrm{Ab}$ & $3,690 \mathrm{z}$ & $3,61 \mathrm{BA}$ & $3,88 \mathrm{~B} z$ & $4,13 \mathrm{Az}$ \\
\hline & 2 & $4,25 A b$ & 3,6306 & $2,74 B C$ & 3,37 Ab & $3,42 \mathrm{Ab}$ \\
\hline & 3 & 3,16 Ad & $1,85 L$ & 2,15010 & $2,29 \mathrm{AL}$ & $2,4 B A C$ \\
\hline & 4 & $3,70 \mathrm{AC}$ & 3,1916 & 3,1686 & $3,4 l A b$ & $3,280 \mathrm{c}$ \\
\hline \multirow{4}{*}{ 罂 } & 1 & $0,35 \mathrm{AL}$ & $0,32 \mathrm{Ac}$ & $0.15 \mathrm{Ad}$ & $0,49 A \mathrm{~A}$ & $0,281 \mathrm{c}$ \\
\hline & 2 & $0,45 B b$ & $0,48 A B D$ & $0,50 \mathrm{AC}$ & $\mathrm{D}, 5 \mathrm{SAb}$ & $0,446 b$ \\
\hline & 3 & $9,79 \mathrm{a}$ & $0,67 \mathrm{Ca}$ & $0,79 \mathrm{Aa}$ & $9,7 \mathrm{SAa}$ & $0,719 \mathrm{Z}$ \\
\hline & 4 & $0,74 \mathrm{Aa}$ & $0,688 \mathrm{~A}$ & 0.76 Ab & $0,76 \mathrm{Aa}$ & $0,69 \mathrm{da}$ \\
\hline \multirow{4}{*}{ 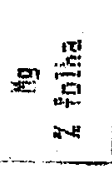 } & 1 & $0,31 B C$ & $0,44 \mathrm{Ad}$ & $0,45 \mathrm{AC}$ & $0,57 \mathrm{AC}$ & $4,279 d$ \\
\hline & 2 & $0,48 \mathrm{Cb}$ & $0,5 \angle B C$ & 0, , LAB & $9,73 \mathrm{Ab}$ & $0,37 \mathrm{Bc}$ \\
\hline & 3 & $0,52 \mathrm{Ba}$ & $0,65 \mathrm{Aa}$ & $0.68 \mathrm{~A} A$ & $0, B Q A=$ & $0,4316 \mathrm{~b}$ \\
\hline & 4 & $0,53 \mathrm{Ca}$ & $0,601 b$ & $0,67 \mathrm{AB}$ & $0,7: \mathrm{Ab}$ & $9,498 \mathrm{a}$ \\
\hline \multirow{4}{*}{ 돌 } & 1 & $3958 \mathrm{c}$ & $593 \mathrm{Az}$ & 478AL & 20615 & $711 A \mathrm{~A}$ \\
\hline & 2 & 55186 & 5123 五 & $6 e^{4} A b$ & $1776 \mathrm{~b}$ & $547 \mathrm{Aa}$ \\
\hline & 3 & 67ذAA & 53683 & $695 \mathrm{Az}$ & $1899 \mathrm{z}$ & $697 \mathrm{Ab}$ \\
\hline & 4 & 5YSAb & 487B & $591 \mathrm{Ab}$ & 1878z. & SMIAC \\
\hline \multirow{4}{*}{ 焉 } & 1 & $326 A 8$ & 242Ab & $217 \mathrm{AC}$ & $263 \mathrm{Ab}$ & $2 d 2 \mathrm{Ah}$ \\
\hline & 2 & 5596 a & $714 A B \mathrm{~A}$ & 810Aa & $735 \mathrm{~A} A$ & b81A \\
\hline & 3 & $669 \mathrm{Aa}$ & 73.AА & $44 i \mathrm{Ab}$ & 76na & $5978 \mathrm{a}$ \\
\hline & 4 & $2 L^{5} \mathrm{Ab}$ & $23 b A b$ & $279 \mathrm{AC}$ & $282 \mathrm{Ab}$ & $238 \mathrm{Ab}$ \\
\hline
\end{tabular}

1 - 0s espacos en branco referenl-se aे interaröes nắ0 significat ivas

2- As letras maibsculas biferentes indicam diferencas significativas na hor izontal (TUKEY 5\%)

3-As letras minfsculas diferentes indican diferencas significativas na vertica! (TUXEY 5\%) 
cont inuąão

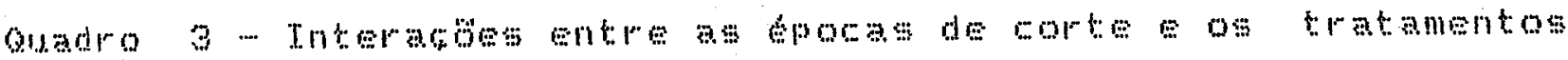

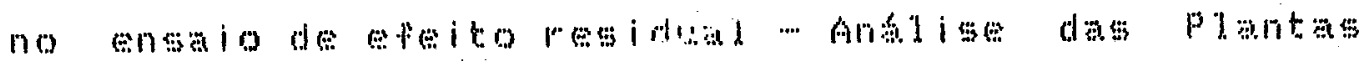
(Capim boloniso)

\begin{tabular}{|c|c|c|c|c|c|c|c|}
\hline \multicolumn{2}{|c|}{ EPOCA } & LE & $L R$ & 9 & 1 & 2 & 3 \\
\hline \multirow[b]{2}{*}{ ז' } & 1 & $10,658 \mathrm{a}$ & $\{9,92 \mathrm{Az}$ & 7, 61Ca & $15,14 \mathrm{Ba}$ & 18,144a & $19,67 \mathrm{Aa}$ \\
\hline & $\begin{array}{l}2 \\
3 \\
4\end{array}$ & $\begin{array}{l}7,4886 \\
3,5006 \\
2,6960\end{array}$ & $\begin{array}{r}11,97 \mathrm{Ab} \\
5,74 \mathrm{AC} \\
4,65 \mathrm{Ad}\end{array}$ & $\begin{array}{l}4,840 \mathrm{~b} \\
3,3 \mathrm{ACc} \\
3,19 \mathrm{BC}\end{array}$ & $\begin{array}{l}6,95 \mathrm{Cb} \\
4,02 \mathrm{BCC} \\
3,50 \mathrm{Alc}\end{array}$ & $\begin{array}{c}10,800 \mathrm{~b} \\
4,078 \mathrm{c} \\
4,60 \mathrm{All} \mathrm{c}\end{array}$ & $\begin{array}{r}16,32 \mathrm{Ab} \\
4,21 \mathrm{AC} \\
4,4 \mathrm{GAd}\end{array}$ \\
\hline \multirow{3}{*}{$\geq$} & $\begin{array}{l}1 \\
2\end{array}$ & 2, $20 \mathrm{AB}$ & $2,023 \mathrm{a}$ & 1,510a & $1,78 C z$ & $2,34 \mathrm{Bz}$ & $2,76 \mathrm{Aa}$ \\
\hline & $\begin{array}{l}2 \\
3\end{array}$ & $\begin{array}{l}1,08 \mathrm{AC} \\
1,01 \mathrm{AC}\end{array}$ & $\begin{array}{l}1,04 A C \\
1,0 \mathrm{BAC}\end{array}$ & $\begin{array}{l}1,93 C \mathrm{C} \\
1,63 \mathrm{C}\end{array}$ & $\begin{array}{l}1, Q Q A B \\
1,11 A C\end{array}$ & $\begin{array}{l}1,1, \mathrm{AB} \\
1,0, \mathrm{AABC}\end{array}$ & $\begin{array}{l}1,32 \mathrm{Abc} \\
1,69 \mathrm{Ac}\end{array}$ \\
\hline & 4 & $1,18 \mathrm{Ab}$ & $1,24 \mathrm{Ab}$ & $1,22 \mathrm{Ab}$ & i, 1.2Ab & $1,22 \mathrm{Ab}$ & $1,16 \mathrm{Ab}$ \\
\hline \multirow{4}{*}{ a. } & 1 & $0,37 \mathrm{Ab}$ & 0,5680 & 0,4605 & 0,501 & Q, $80 \mathrm{AB}$ & $0,95 \mathrm{AG}$ \\
\hline & 2 & $9,67 \mathrm{AC}$ & $0,68 \mathrm{AC}$ & $0,73 \mathrm{Ab}$ & $9.69 \mathrm{ABC}$ & $0,69 \mathrm{Add}$ & $0,65.9 \mathrm{c}$ \\
\hline & 3 & 0,8206 & $0.93 \mathrm{AO}$ & $0,890 \mathrm{~b}$ & $0,3 \mathrm{BA} A \mathrm{~d}$ & $0,93 \mathrm{Ab}$ & $0,79 \mathrm{AMJ}$ \\
\hline & 4 & $1,92 \mathrm{~B}$ & $1.19 \mathrm{Aa}$ & $0,98 B a$ & $1,10 \mathrm{Aa}$ & $1,16 A \mathrm{a}$ & $1,18 \mathrm{Aa}$ \\
\hline \multirow{4}{*}{ 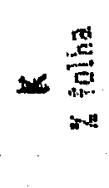 } & 1 & $3,699 b$ & $4,93 \mathrm{Az}$ & $4,68 \mathrm{AB}$ & $3,67 \mathrm{~A}$ a & 3.910 a & 3.0732 \\
\hline & 2 & $2,87 \mathrm{BC}$ & $3,93 \mathrm{Ab}$ & $4,85 \mathrm{Aa}$ & $3,15 t h$ & $2,86, \mathrm{Cb}$ & 2,7206 \\
\hline & 3 & $2,33 \mathrm{Ad}$ & $2,43 \mathrm{Ad}$ & $3,19 A C$ & $2,39 \% \mathrm{c}$ & $2,11 \mathrm{Cr}$ & $1,06 \pi, \mathrm{C}$ \\
\hline & 4 & $3,48 \mathrm{Aa}$ & $3,24 \mathrm{Bc}$ & 4,07 Ab & $3,458 \mathrm{a}$ & 3,6506 & $2,880 \mathrm{C}$ \\
\hline \multirow{4}{*}{ 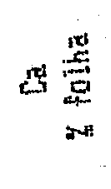 } & 1 & $0,39 \mathrm{Ad}$ & $0,308 c$ & $0,36 \mathrm{Ac}$ & $0,327 \mathrm{c}$ & $0.74 \mathrm{ABC}$ & $0,34 \mathrm{AMC}$ \\
\hline & 2 & $0,52 \mathrm{AC}$ & 0,4386 & $0,44 B 6$ & $0,52 \mathrm{AG}$ & $0,50 \mathrm{Ab}$ & 6,4686 \\
\hline & 3 & $0,78 \mathrm{Az}$ & $0.703 \mathrm{a}$ & $0,641 \mathrm{a}$ & $6,7 B A z$ & $9,7 \mathrm{BAZ}$ & $0,76 \mathrm{Aa}$ \\
\hline & 4 & $0,75 \mathrm{Ab}$ & $0.71 B a$ & $0,61 \mathrm{Ba}$ & $0,7 / \mathrm{A} \mathrm{A}$ & $0,75 \mathrm{Aa}$ & $0,75 \mathrm{Aa}$ \\
\hline \multirow{4}{*}{ 焉 } & 1 & $0,44 A C$ & $0.373 \mathrm{~d}$ & $0,29 \mathrm{Cd}$ & 0,4636 & $9,4 \mathrm{BAC}$ & $0,47 \mathrm{Ad}$ \\
\hline & 2 & $0,5 \mathrm{BA} \mathrm{a}$ & $0,52 \mathrm{Bc}$ & $0,38 \mathrm{Cc}$ & $0,64 \mathrm{Aa}$ & $0,63 \mathrm{Ab}$ & 0,5580 \\
\hline & 3 & $0,588 \mathrm{z}$ & 0, BSA & $0,43 C b$ & $9,639 \mathrm{a}$ & 9,79AB & $0,7 \mathrm{BAC}$ \\
\hline & 4 & $0,550 b$ & $0,65 \mathrm{Aa}$ & $9,4 \mathrm{Ba}$ & $0, \angle 2 \mathrm{Aa}$ & G, GGAab & $0,64 \mathrm{Ab}$ \\
\hline \multirow{4}{*}{ 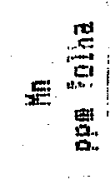 } & 1 & 438Ab & 477Ad & $312 \mathrm{Ca}$ & $410 B i$ & $495 \mathrm{BC}$ & $617 \mathrm{Ad}$ \\
\hline & 2 & $583 \mathrm{Az}$ & $541 A C$ & $337 \mathrm{Ca}$ & $572 \mathrm{Ba}$ & $\angle 37 A B a, b$ & $700 \mathrm{AC}$ \\
\hline & 3 & $5698 a$ & 717Aa & 7330 a & $544 C z$ & 6960 & $1696 \mathrm{AB}$ \\
\hline & 4 & $469 \mathrm{Bb}$ & $655 \mathrm{Ab}$ & 3150 & $453 \mathrm{Cb}$ & 506 b & $874 A b$ \\
\hline \multirow{4}{*}{ 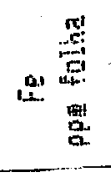 } & 1 & $2544 h$ & $271 \mathrm{AO}^{\circ}$ & $300 \mathrm{~A}$ & $283 \mathrm{Al}$ & 2\{\{Á\} & $256 \mathrm{Ab}$ \\
\hline & 2 & $5496 \pi$ & B75A2 & $700 \mathrm{AB}$ & $732 \mathrm{AB}$ & $733 \mathrm{AB}$ & GULA \\
\hline & 3 & $5792 \mathrm{a}$ & $795 \mathrm{AB}$ & 627ka. & BGSA & 6697 a & $5901 \mathrm{a}$ \\
\hline & 4 & 197Ab & $323 \mathrm{Ab}$ & $240 \mathrm{Ab}$ & $287 \mathrm{Ab}$ & $266 \mathrm{Ab}$ & $247 \mathrm{Ab}$ \\
\hline
\end{tabular}

1 - Os espacos en branco refeream-se a interacôes näo signi?icat ivas

2- As letras mailisculas diferentes indican diferencas significativas na hor izontal (TUKEY 5\%)

3- As letras aindsculas diferentes indican diferencas significativas na. vertical (TUKEY 5\%) 


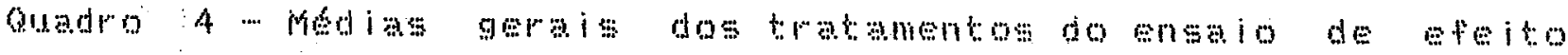

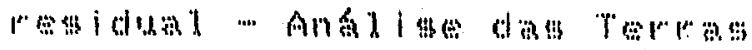

$P A R A M E T R D$

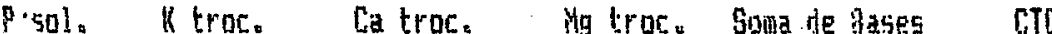

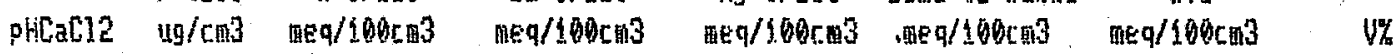

$5-514$

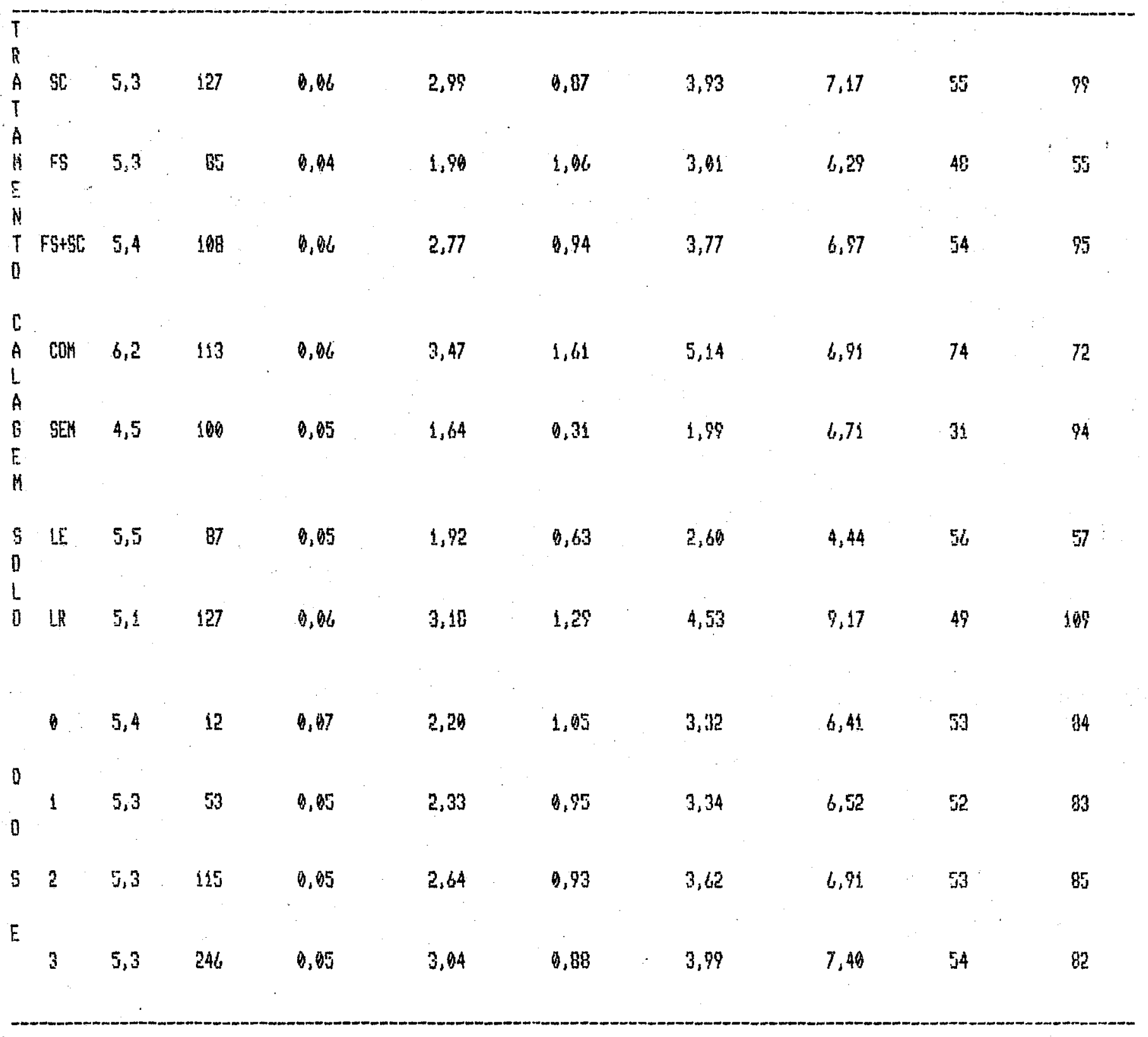




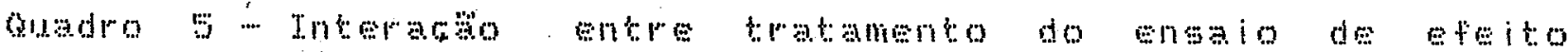

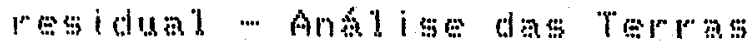

50LO CALABEH DOSE

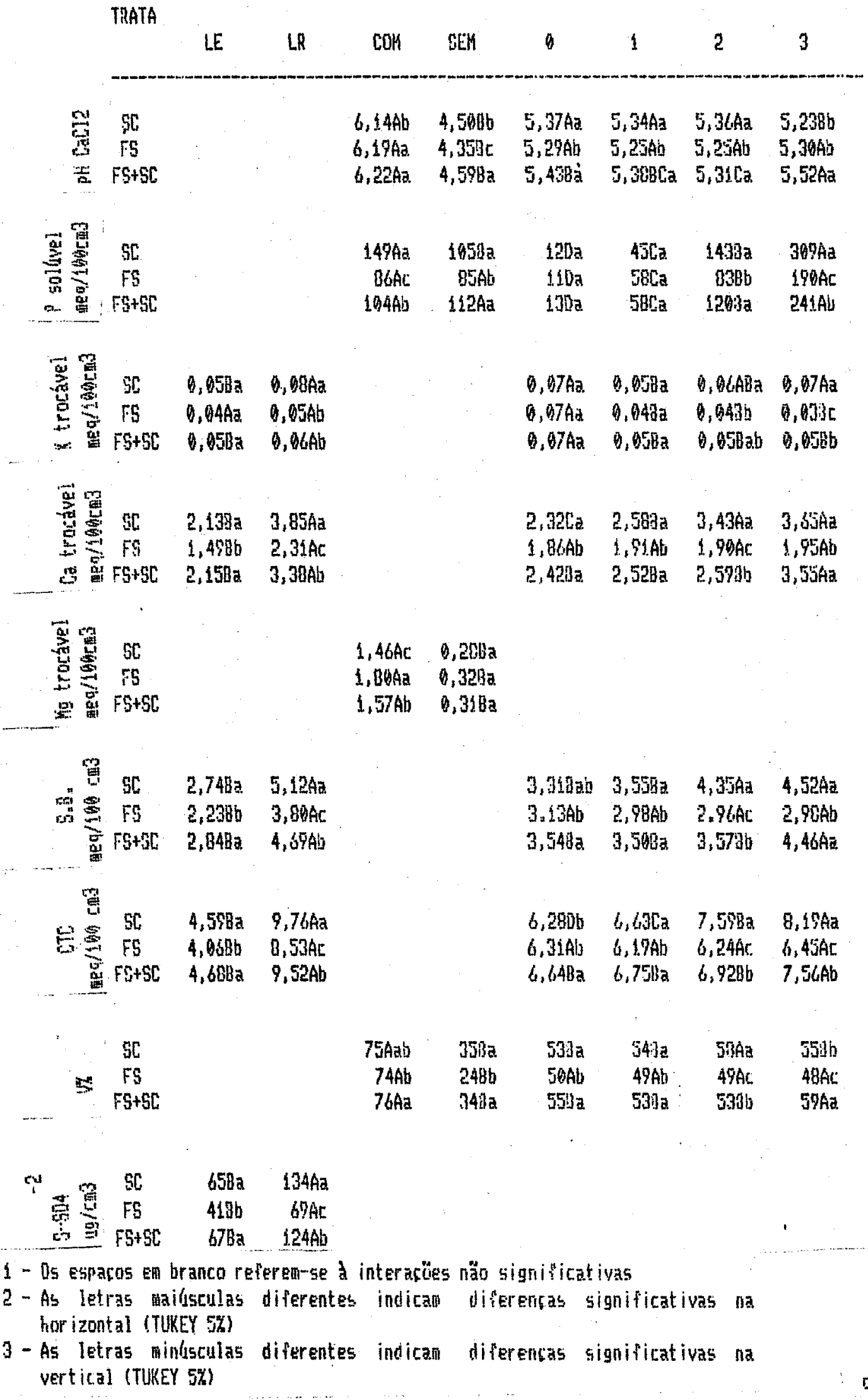


tontinuarăa

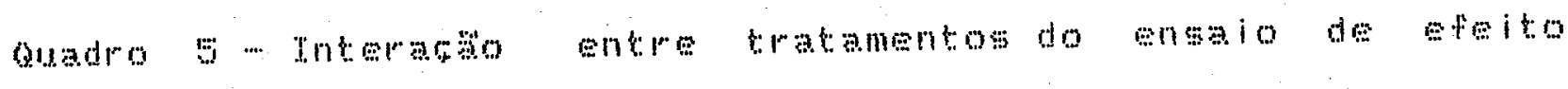

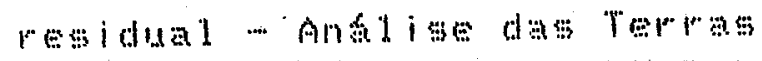

\begin{tabular}{|c|c|c|c|c|c|c|c|}
\hline & \multirow{2}{*}{5010} & \multicolumn{2}{|c|}{$C A \perp A B E H$} & \multicolumn{2}{|c|}{005} & \multicolumn{2}{|l|}{$E$} \\
\hline & & $\mathrm{COH}$ & SEK & 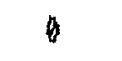 & 1 & 2 & 3 \\
\hline . 畐 & LE & $6,49 \mathrm{Aa}$ & $4,608 \mathrm{~Pa}$ & & & & \\
\hline 5 & Ln & $5,88 \mathrm{AB}$ & 4,3796 & & · & & \\
\hline 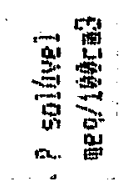 & $\begin{array}{l}\text { LE } \\
\text { LR }\end{array}$ & & & $\begin{array}{r}90 a \\
1.50 a\end{array}$ & $\begin{array}{l}4 \pi \mathrm{Ch} \\
64 \mathrm{Ca}\end{array}$ & $\begin{array}{l}1036 b \\
1289 \mathrm{a}\end{array}$ & $\begin{array}{l}193 \mathrm{Ab} \\
396 \mathrm{Aa}\end{array}$ \\
\hline 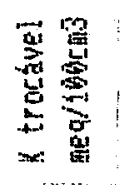 & $\begin{array}{l}\text { LE } \\
L R\end{array}$ & & & $\begin{array}{l}0,00 \mathrm{Ab} \\
0,97 \mathrm{Aa}\end{array}$ & $\begin{array}{l}0,04 \mathrm{Aa} \\
0,65 \mathrm{in}\end{array}$ & $\begin{array}{l}0,65 \mathrm{Aa} \\
0,058 \mathrm{a}\end{array}$ & $\begin{array}{l}0,05 \mathrm{~A} \\
0,053 \mathrm{a}\end{array}$ \\
\hline 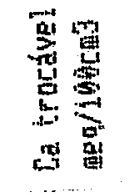 & $\begin{array}{l}\text { LE } \\
\text { LR }\end{array}$ & $\begin{array}{l}2,51 \mathrm{Ab} \\
4,43 \mathrm{Az}\end{array}$ & $\begin{array}{l}1,358 \mathrm{~b} \\
1,948 \mathrm{a}\end{array}$ & & & & \\
\hline 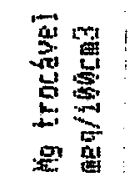 & $\begin{array}{l}\text { LE } \\
\text { LR }\end{array}$ & $\begin{array}{l}1.92 \mathrm{Ab} \\
2,20 \mathrm{Aa}\end{array}$ & $\begin{array}{l}0,236 \mathrm{~b} \\
0,393 \mathrm{a}\end{array}$ & & & & \\
\hline c & $\begin{array}{l}\text { LE } \\
L R\end{array}$ & $\begin{array}{l}3,59 \mathrm{Ab} \\
6,70 \mathrm{Aa}\end{array}$ & $\begin{array}{l}1,62 \mathrm{Bb} \\
2,379 \mathrm{a}\end{array}$ & & & & \\
\hline 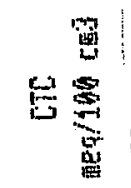 & $\begin{array}{l}\text { LE } \\
L \pi\end{array}$ & $\begin{array}{l}4,64 \mathrm{Ab} \\
9,19 \mathrm{AA}\end{array}$ & $\begin{array}{l}4,260 \mathrm{~B} \\
9,17 \mathrm{Aa}\end{array}$ & & & & \\
\hline & LE & 77Aa & $36 \mathrm{Ba}$ & & & & \\
\hline 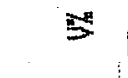 & $L R$ & 73Ab & 2690 & & & & \\
\hline 蚛要 & $\begin{array}{l}\text { LE } \\
\text { IR }\end{array}$ & & & . & . & & \\
\hline
\end{tabular}

1 - Ds espacos en branco refereat-se a interarîes não significatiyas

2 - As letras mailsculas diferentes indicam diferencas significativas na. hor izontal (TUKEY 5\%)

3- As letras ginksculas diferentes indican diferencas significativas na vertical (TUKEY 5X) 
cunt inuaçัด

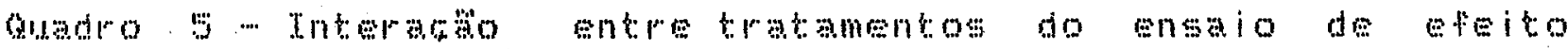

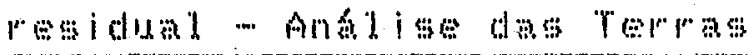

\section{1) $\$ E$}

CALAGEYI

$12 \quad 2 \quad 3$

\begin{tabular}{|c|c|c|c|}
\hline$\frac{r y}{y}$ COAf & $6,27 \mathrm{Aa}$ & $4,200 \mathrm{~b}$ & $6,14 \mathrm{BCa}$ \\
\hline SEH & $4,493 \mathrm{~b}$ & $4,44 B b$ & $4,470 \mathrm{~b}$ \\
\hline
\end{tabular}

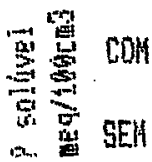

\begin{tabular}{|c|c|c|c|}
\hline 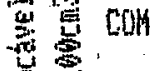 & $0,07 \mathrm{Aa}$ & Q, $B \angle A B B$ & $0.05 \mathrm{Ba}$ \\
\hline 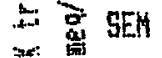 & $0,07 \mathrm{Aa}$ & $9,048 \mathrm{~b}$ & $0,043 \mathrm{a}$ \\
\hline
\end{tabular}

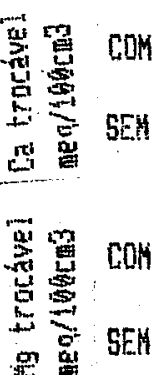

点兽 COM

COH 76Aa 74Aa 74Aa . 74Aa

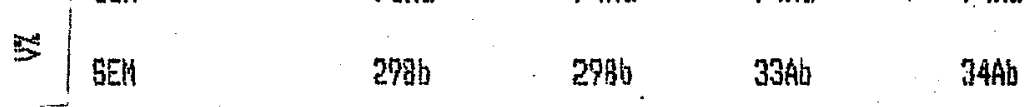

ru

$+2 \mathrm{COH}$

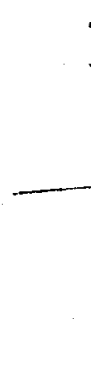

$$
\text { S COM }
$$

它

tr. 믁 SEM

1 - Ds espacos en branco refereat-se a interacöbs näo significativas

2 - As letras maibsculas diferentes indican díferencas significativas na hor izontal (TUKEY 5\%)

3-As letpas mindsculas diferentes indicam diferencas significativas na. vertical (TUKEY 5X) 


\subsection{Ensaio com micronutrientes}

linimiamente vamos apresentar o eleito dos

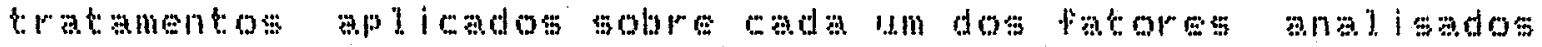
isoladnmente para as duas culturas

Wume segunda parto do discusato, and 1 isaremos

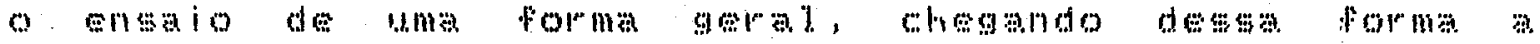
conclusoses ma is abrangentes"

4. 2. 1. Apresentáño dos resultados

$$
4.2,1.1 . \operatorname{col} z a
$$

A colsa foi cultivada num primeiro periodo, do $19 / 06 / 86$ a 27/08/86, a o efeito dos ratamentos foi o seguince cos resuladog dos partatros avaliados esta no

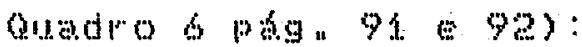

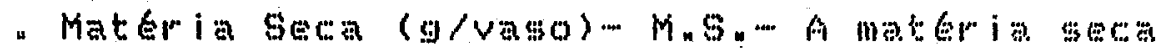

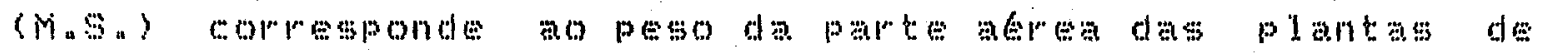
mada vaso, secas em estuta Anal isando os dados do Quadro 6 observang que a aplicaca

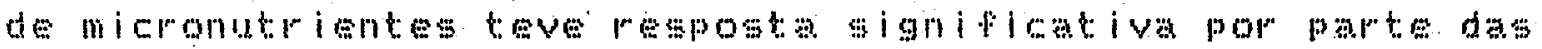
plantas, uma vez que nom fratomentogem que eles foran

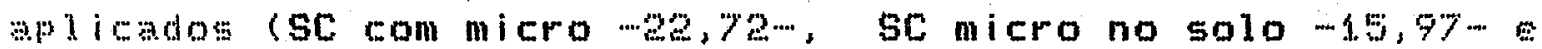




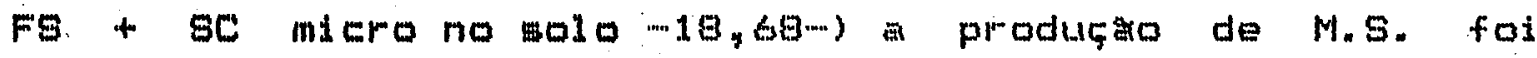

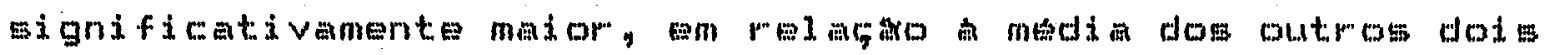
tratamentos $-10,42$. A maior producto ocorreu com a aplicaço dos iniconutrientes dentro do $\mathrm{SC}$

- Nitmogenio (\% na parte armea $-N-0$ tior de N\% na prte area teve iqual comportamento em todos os tratamentos em que os nutrimontes form aplicados via SC. Apenas no tratamento Fs + SC miero no solo houve na dose 2 un incmemto significativo do teor de nitrogenio, passando de 1,49 (media dos outmos tratamentog) para $2,22$.

De qualquer forma o teor de nitrogenio foi pouco afetado pelos tratamentos ou pelas doses, apesar. do Valor significativo de "F" para tratamentos e para a interaço tratamentos $x$ doses:

- Fosforo (\% na parte merea) - F - o teor de fosforo na parte aerea motrcu-se bastante variavel.

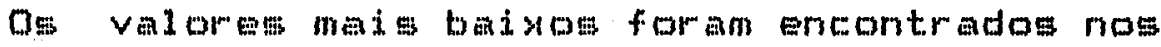
tratamentos SC micro no respectivamente. Us tros tratamentos restantes apresentaram Valores mais eleVados de F, mesmo o fratamento FS + GC sem mi cro. 


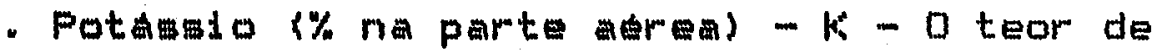
pot a seu comportamento mostra as mesma tendencias do fosforo. o Valor medio de 2,62 nos cinco tratamentos básicos.

- Calcio (\% na parte aprea) - Ca - O teor de Ealcio năo apresentou variacoro en melaço aos tratamentos ou m. doses. nas mendo afetado pela forma de aplicaço dos micronutrientes. 0 valor medio basicos.

- Magnesio (\% na parte aprea) - Mg - o teor de magnesio tamben nao apresentou multa variagas, apenas nos

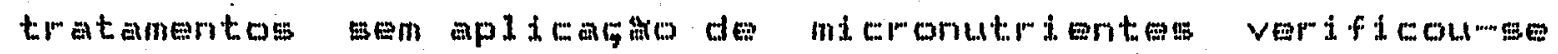
Valores significativamente inferiores, 0,16 em media nos tratamentos sem micronutrientes e 0,23 naqueles em que houve a aplicaça.

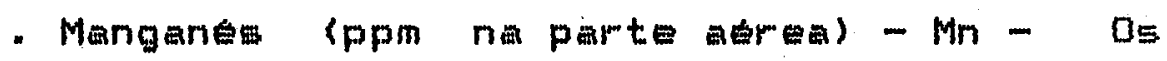

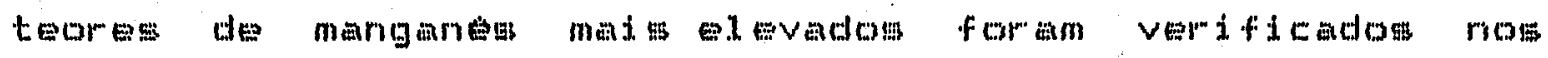
tratamentos em que ow fertilizontes fom aplicados na forma

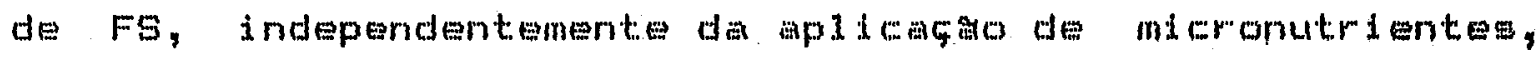

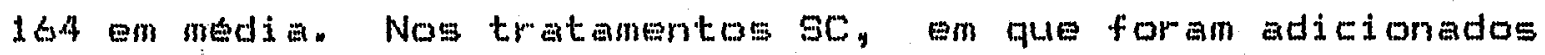
micronutpientes o teor de manganes foi mais alevado. 


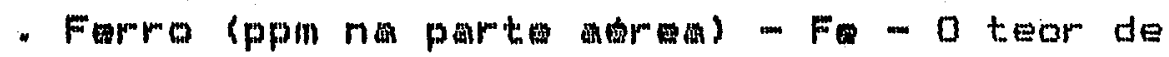
fermo mpresenta Ou valores maje elevados concentram-ge nos tratamentos fos (168 em media) os mais beixos nos tomtamentom SC (b6 em motial.

\subsubsection{Arrma}

o arroz foi cultivado num segundo periodo, de $15 / 10 / 86$ a $20 / 01 / 97$ com reaplicacio de meia dose de $N_{y} P$ e $K$ sendo o efeito dos tratamentos sobre os parametros avaliados, o seguinte fom resultados numericos dos parbmetros avaliados esta no amaro 7 pag. 93 e 94).

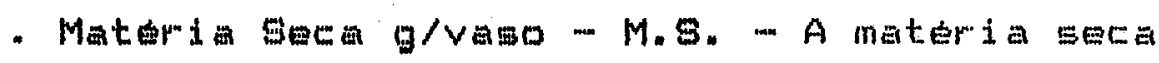
$\left(M_{n} S_{n}\right.$ corresonde a peso da parte aprea das plantas de cada vaso secas em estufa a 60 o ats peso constante. Os

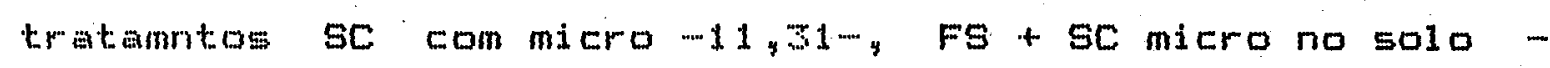
$11,35 m-F S+5 C$ sem micro -12,19-, apresentaram o mesmo desempento. Ds dois tratamentos restantes, SC micro no solo e SC sem miero, tiveln uma productom de materia sera significativamente menor, 9,31 em media. Na dose 20 desempentio dag plantas foi superior, nao havndo interacá entre as doses os tratamentos. 
- Nitrogenio (\% na parte mera $)-N-D s$

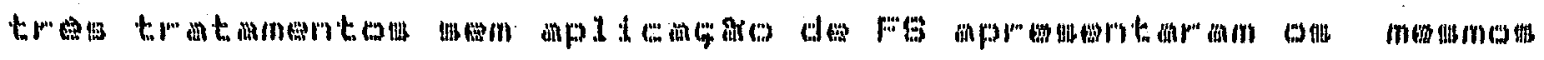
valores de N\% na parte aerea congiderando os tratamentos em

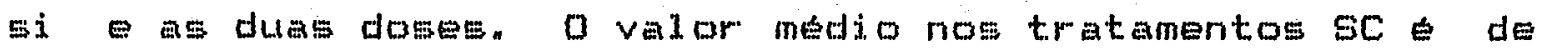
0,90 em rela50 a 1,00 nog tratamentos Fs. Nos tratamentos com aplicacar de FS a dose 2 esteve assoeiada a Valores mais elevados de N\%" Comparando-se os tratamentos, houve pouca Variaço, gendo os Valores battante uniformes.

- Fomforo \{\% no parte mpea - P - Da mesma maneira como para o nitrogenioy os tres tratamentos com aplicacaro do N, F eKi via BC, se comportaram de forma identican Entmetanto, o teror de p noe tratamentos com aplicacolo de FG foi significativamente maior, neseses dois tratamentos, mose 2 mpresentou Valomes tambem superiores. Q Valor medio dos tratamentom SC de 0,44 dos tratanentos $F, 0,81$.

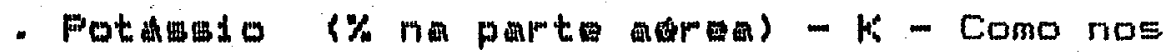
dois casos anterioress os tros tretamentos com aplicato de

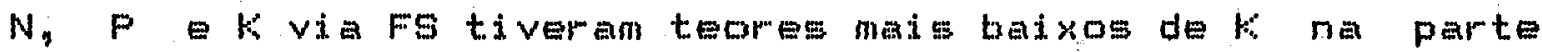

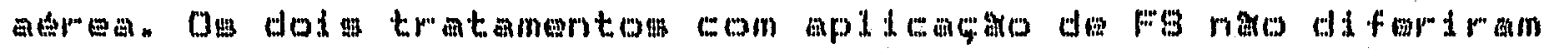
entre si. Nia dose 2, mom todos os casom, o valor dek foi superior" Naro foi verificada interacas signdficativa entre as doses tratamentos. O val or medio dos tratamentos sC 3,03 e dos tratanentos $\mathrm{FG}, 3,90$. 
- Calcio \{\% na parte aprea - Ca - O tratamento sc sem miero aprementou o valor mais elevado de Ca $(0,91)=0 F 5+5 C$ micro no solo o mas baiso $(0,61)$. OS tres tratamentos petantes apresentaram valores intermediarios e semelhantes entre Ca ma parte amea foi superior. Nä houve interaço significativa eritre os tmatamentose as dosem. De uma maneila germ a variaco dos valores now e muito grande e nă segue uma tendencia bem definida em relacaso abs tritamentos.

" Magriesio (\%, na parte a de Mg foi Eignificativamente superior nos tres tratanentos en que os nutrientes $N, F$ fo foram aplicados via scy sendo. gue os Valores, dentro deswestratamentos sao semelhantes 10,32 em medi

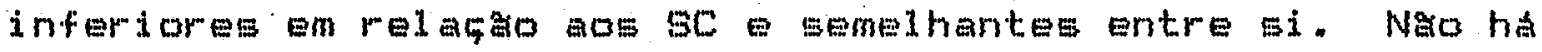

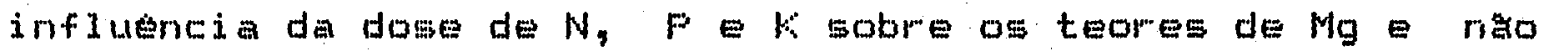
ha tamben interaço significativa entre tratamentos doses.

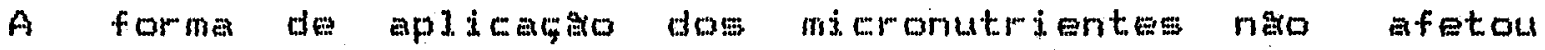
sigrificativamente os teores dk Mg. O teor de Ma, bem como no Easo do $P$ E fol Influmeiado mai pela forma de aplicacă dos nutrientes N, $F$ é (via SC ou via FS).

- Mangunes (ppm na parte ampal - Mg - Ds tratamentos SC com micm e SC micmo no solo apmesentaram os 
valores mais elevado de Mn, 964 e 989, mepectivamente.

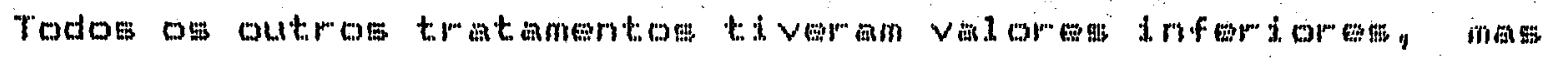

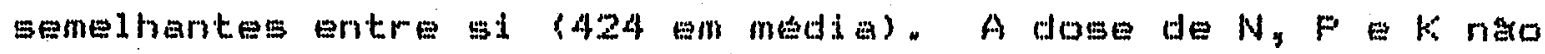
apresentou feito sobre os Valores de Mr e tambem nâ foi verificada interaço gignificative entre doses e tratamentos. A forma de aplicaso dos micronutrientes parece tamben nao ser a causa da variaca dos resultados.

- Ferro (ppm na parte aprea) - Fe - o teor d Fe bastante unifome nos tratamentos. Todos os

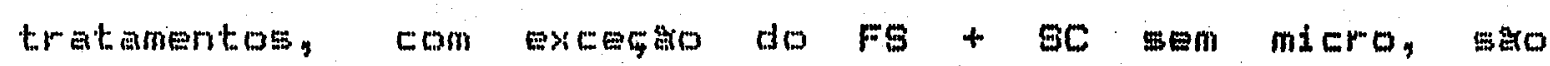
semelhantes entre 91 (205 em media). Nestes tratamentos

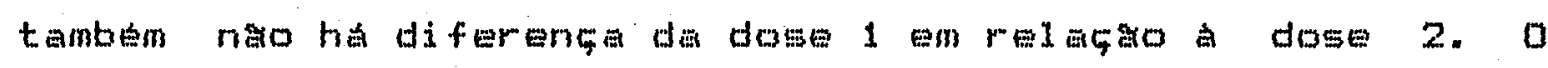
teor de Fe no tratamento FS+SC an micmo no dome 1 apresentou Lim valor significativamente maior do que todas os demais (513). A forma de aplicara dos micronutrientes parkeg tambem nao influenciar o teor de Fe na parte aerea.

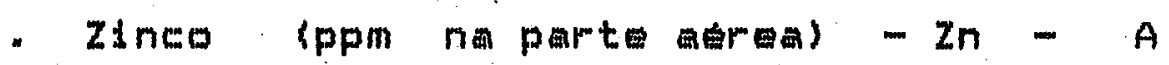
aplicaco de inderonuturentes teve influmeda mignificativa no teor de Zn das plartas, lumia vez que en todos os

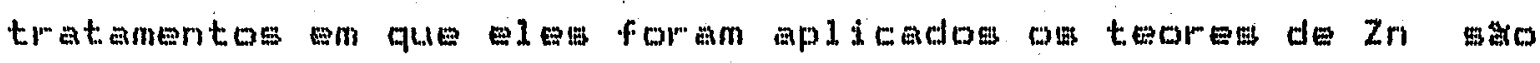
significativamente maiome mas semelhantes entres 1119 em medial. Nos dois tratamentos sem aplicacoso de mi ronutrientes os valores saro inferiores aos dos outmos

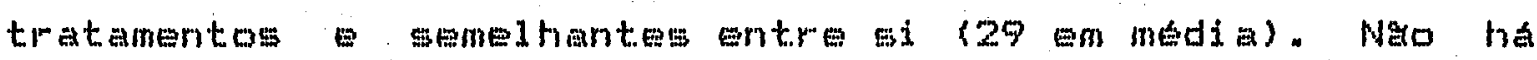


Influenci das dobes de $N_{*} P$ e $K$ sobre o teor de $Z n$ tambem

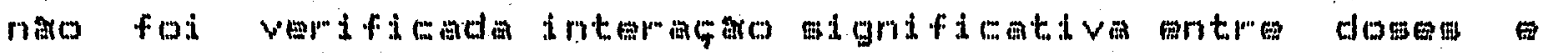
tratamentos. A forma de aplicacto dos micronutrientes parece tamben nơ afetar o teor de $\mathrm{Zn}$ das plantas.

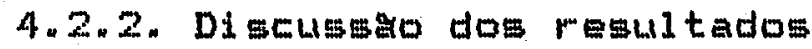

Frar a di

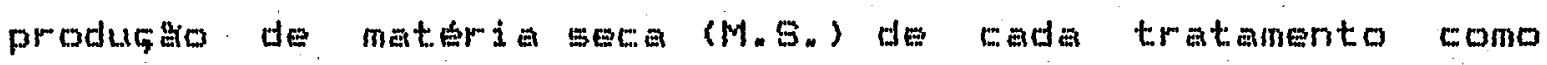

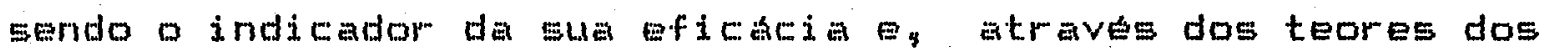
nutrientes na folta, tentaremos explicar o comportamento apresentado.

No primeiro cultivo (cola a $15 / 06$ a $27 / 08 / 96$ ) ficou evidente o efeito significativo. da aplicaço dos micronutyentes, una vez que nos dois tratamentos em que eles naso for am aplicados - $\mathrm{SC}$ sem micmo FS + SC sem microa producto de M. S. foi gignificativamente menor.

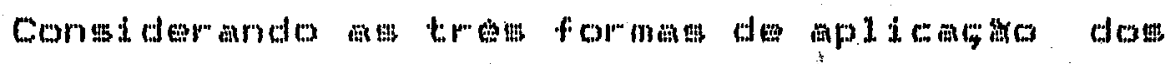
micronutyientes via SC rom micmoy via gC micro no solo e $\mathrm{FS}+\mathrm{SC}$ micro no solo otratimento vili sc com mico apresentou a maior producto de M.S. naro diferindo estatisticamente do tratamento FS + SC micro no solo.

Atraves dia andise do teor de alguns 
nutrientes ne parte an area das plantas (ouadro o) nao foi

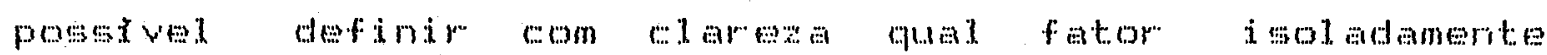
contribuiu Em maior propormo indusindo o comportamento obserman.

Mos dois tratementos de maior. desempento ma

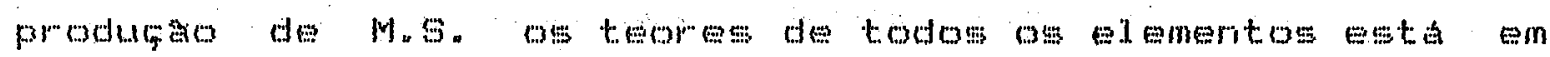
novis elevados, en quase folos os casos apresentando os valores mats elevedos of toclos os tratamentos poren nimo excessivos. O pior desempentio do tratemento SC micro no solo ná pode ser explicado pelos paranetrog analisados.

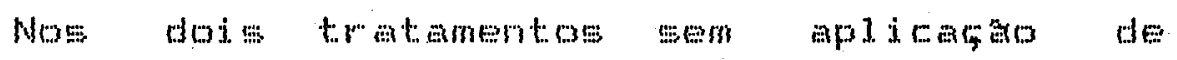

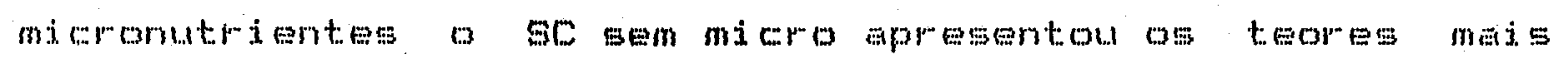
baisos de praticamonte todos os nutmientes analisados, principalmente o ainco, fato gue pode explicam ese comportamento o cutso fratamento sem aplicacos de micronutrientes FG t SO sem micro iopresentou desempento semelhante ao anterior mas toores de mutrientes seme1hantes

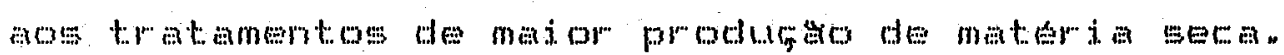

Como principad ats o momento podemos citar quey para as condicbes esperimentals a aplicacino de micronutrimentes teve efeito

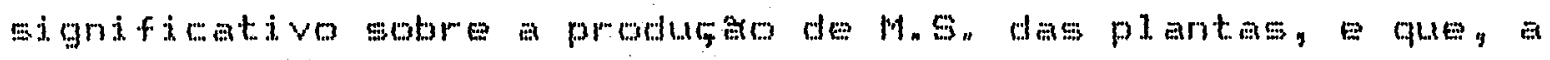
mplicaráto via sc perfeitamente vidvel proportonando 
productao igual ou superior en relagao à aplicacao dos

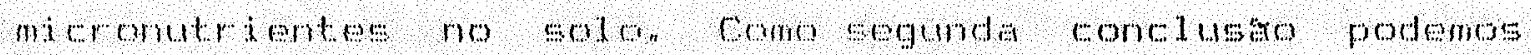
Citar que os parametros aralisados, isto \&, o teor de alguns nutrientes na foltha, nao permitira explicar de maneira definitiva a diferenca de comportamento, existente e observada, dos tratementos.

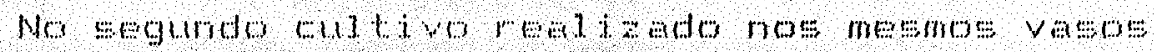
(arros $15 / 10 / 96$ a $20 / 01 / 87)$ a influmela da aplicacta dos micromutrientes ja \& bem menor tridente, uma vez que trat amentos com e sem aplicerso de mi cronutrietes tiverem o mesmo desempention.

o tratanento Fo t $\mathrm{SC}$ anem miero teve productao de M.8. semenharite ar FS t SC micro no solo e SC com micro.

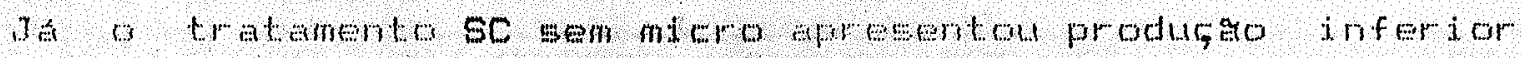
aOs trese anteri ormente cil adres mas eemellante ao SC micro no 5010

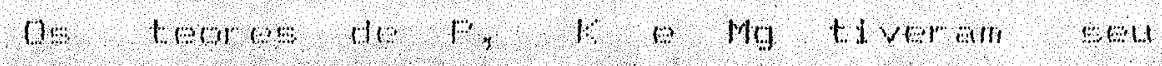

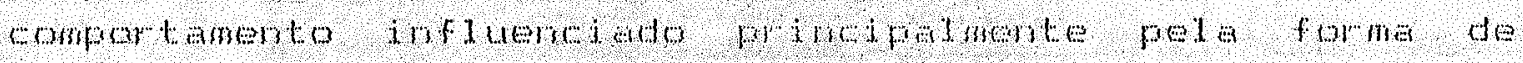

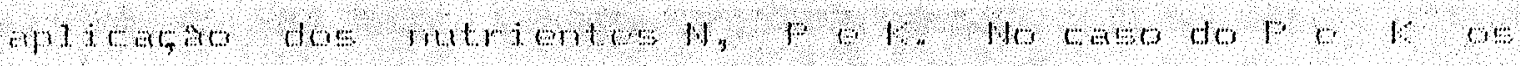

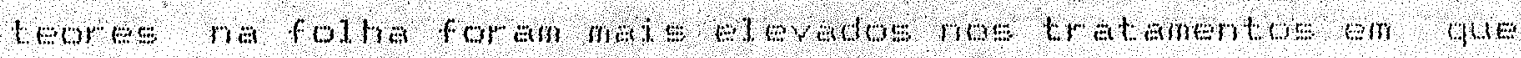
for an utiliztados FS.

a torm desemperito dos tratementhe com 


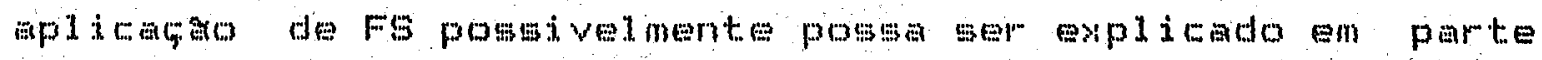

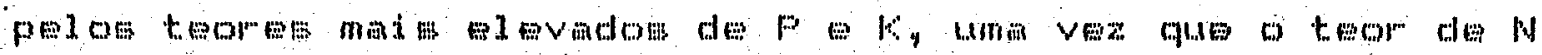

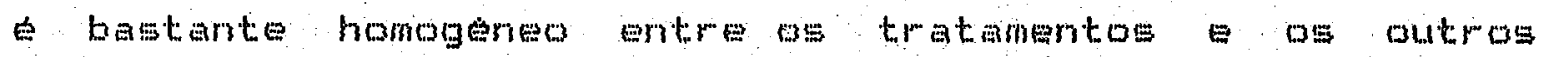

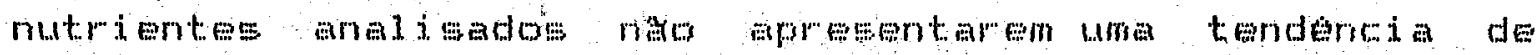
comportamento bem definida.

o teor de zn fol afetado pela aplicacto ou nao de micmonutrientes, sendo os teores de Zn na parte aerea das plarias significativamente inferiores nos

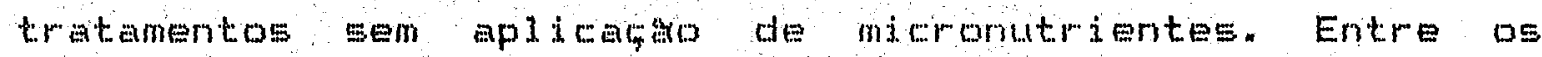
diferentes modos de aplitacto o comportmento foi semel hantion

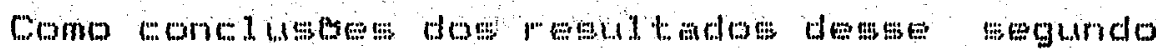
rutivo podemos eiter que ja no evidente o efeito da

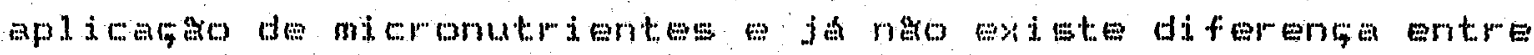

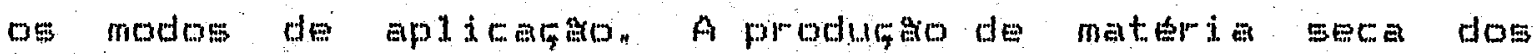
tratamentos, muto ma uniforme se comparada a primeiro cultivo, pode apenas em parte ear expli fada pelos parametros aval i ados.

4. 2.3. Conc1umber do momo

Como conclugbes germ desen eneaio podemos

afirmar que: 
a) a aplicato de micromutientes, via SC

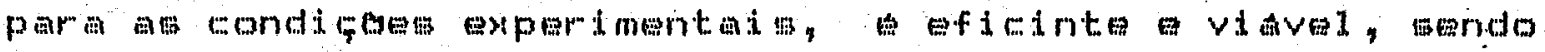
comparavel ou superior a aplicato no nolo:

b) o efeito do molo de aplicasa dos micronutrientes restringemse a um periodo de cultivo. No - Eggundo periolo de cultivo, men reaplicacã dos miconuty isties, 0 comportamento das plantas tende a se tornar ma

c) os parmetros arralisados para explicar as difurentes producbes de materia seca tteor de alguns

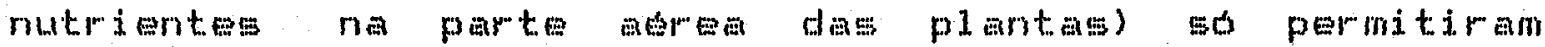
explicacoes partiais. 


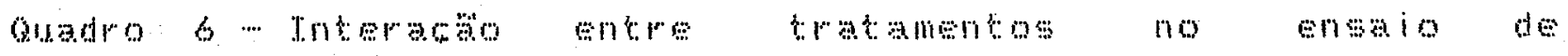

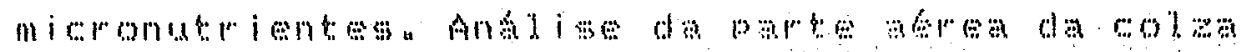

TEOA NA PARTE AEREA

\begin{tabular}{|c|c|c|c|c|c|c|c|}
\hline H. 5. & $N$ & $P$ & K & Ca & $\mathrm{Mg}$ & An & Fe \\
\hline$g$ & 7 & $\%$ & $\%$ & $\%$ & 7 & Pp⿴l & pigh \\
\hline
\end{tabular}

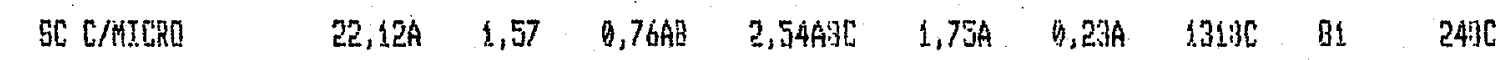

14 SC MICRO SOLO 15,97BC 1,37 Q.6ABC 2,11C 1,5TA 0,24A 139BC 52 30ABC

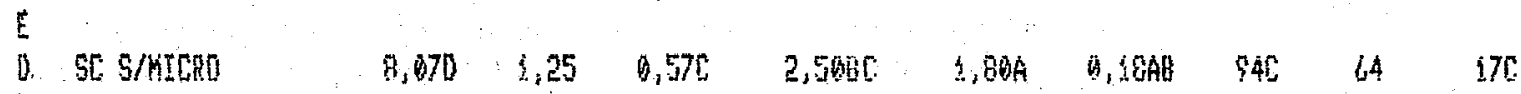

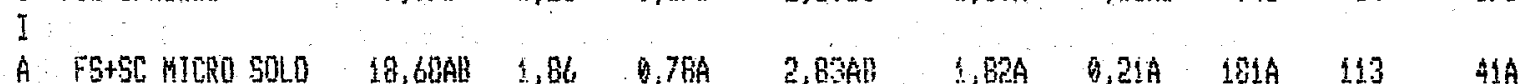

5

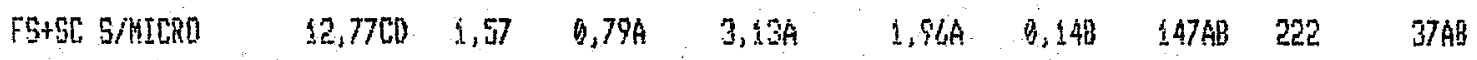

0.4.5. $\quad(5,47) \quad(0,13) \quad(0,62) \quad(0,44) \quad(6,06) \quad(47) \quad(14)$

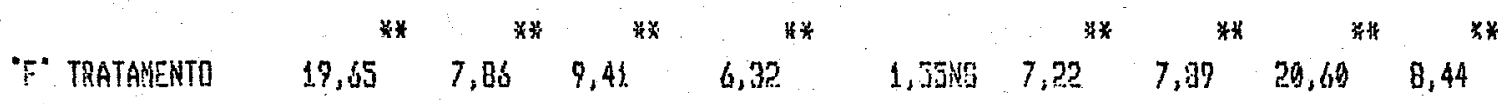

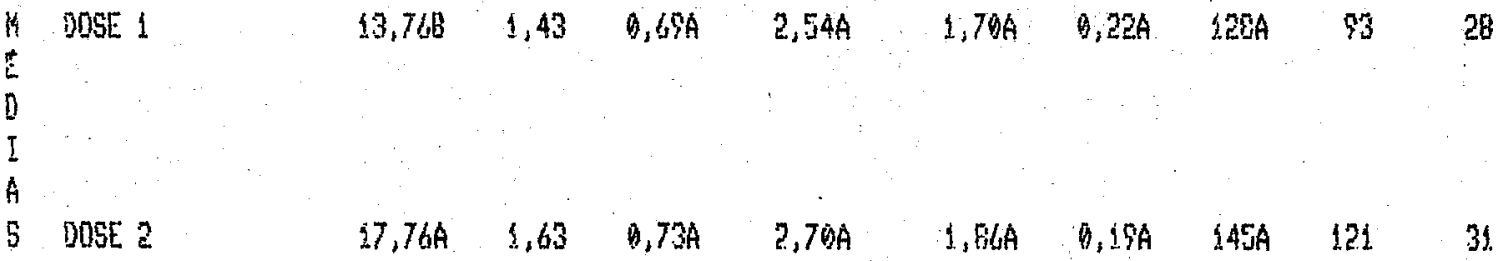

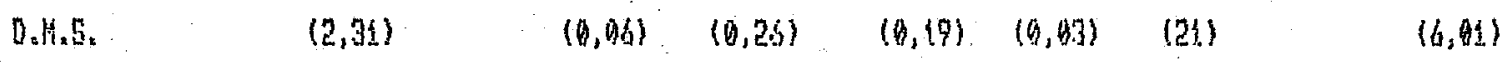

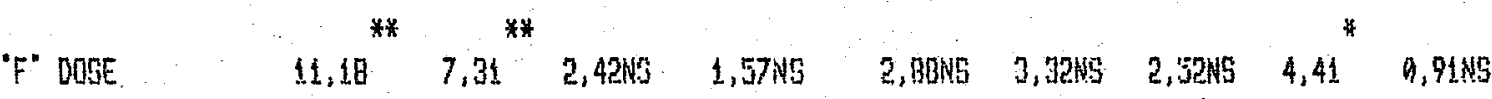

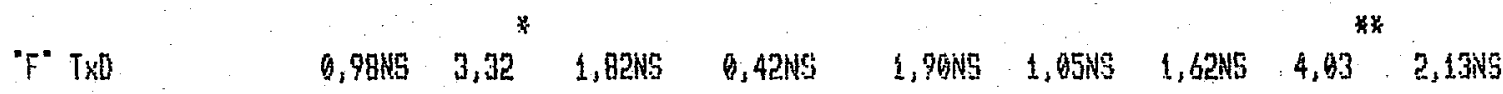

* TUREY IY

TULEY 5\%

continua 
cont inmata

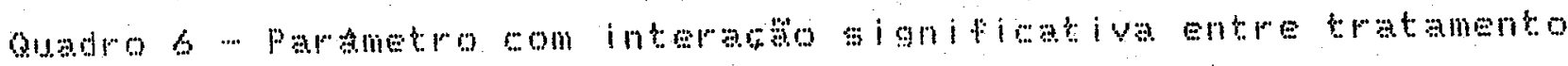
x. dosis

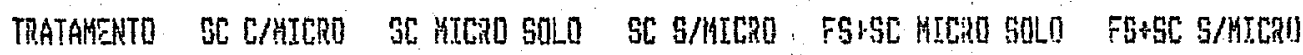
DIjEt

NITROG NIO \%

D.K.S. $(9,40)$ mล i(tsenlo (5\%)

Doge 1

$1,48 \mathrm{AB}$

$1,39 \mathrm{~A}$ ב

1,2BA\%

1,5946

1. $59 \mathrm{Aa}$

D.M.3. (9,34) gindisculo (5\%)

TUREY

$$
1,678 \mathrm{a}
$$

1,438 a

$1,238 \mathrm{a}$

$2,22 A 3$

1,589a

FERR D PPI

DOSE 1

71A32

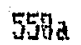

7582

$74 i b$

6. H.5. (87)

坛ifsculo (5x)

0.H.5. (62) Rinúsculo (5\%)

TUKEY

$720 \mathrm{ca}$

$51 \mathrm{ca}$

$540 z$

1521 .

2T5Aa 


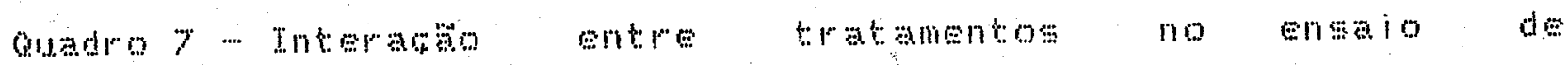

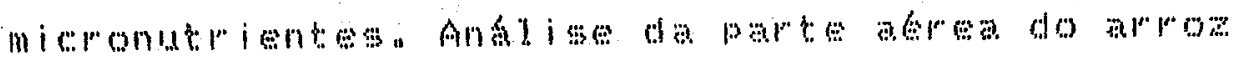

TEOR NA PARTE AEREA

\begin{tabular}{|c|c|c|c|c|c|c|c|c|}
\hline $\mathrm{M} . \mathrm{S}_{\mathrm{B}}$ & $N$ & $P$ & K & $\mathrm{Ca}$ & $M g$ & Pin & $F e$ & $\mathrm{In}$ \\
\hline 9 & $z$ & $y$ & $\%$ & $\%$ & 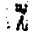 & pell & PpR & PPQ \\
\hline
\end{tabular}

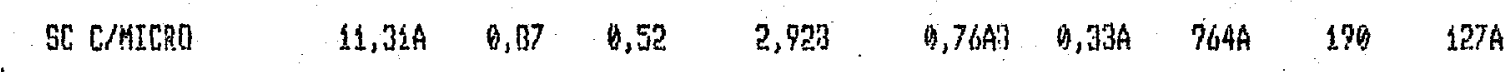

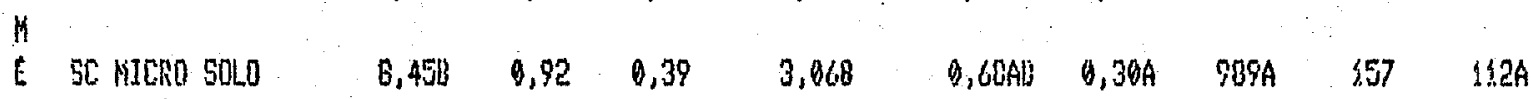

D.

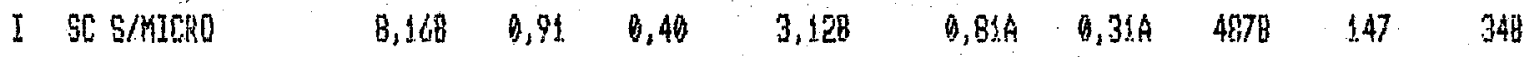

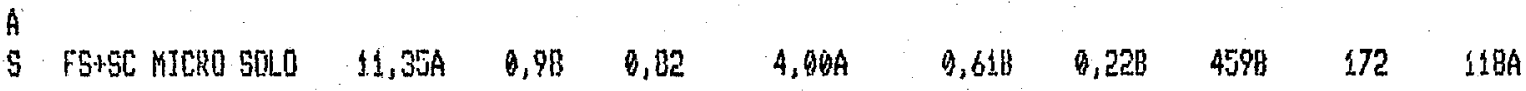

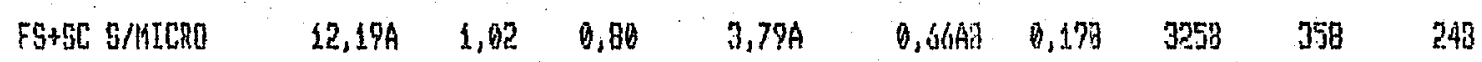
D.H.S.
$(2,62)$
$(0,40) \quad(0,17) \quad(0,05) \quad(207)$
1539

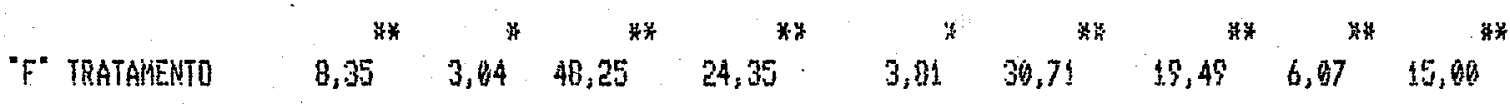

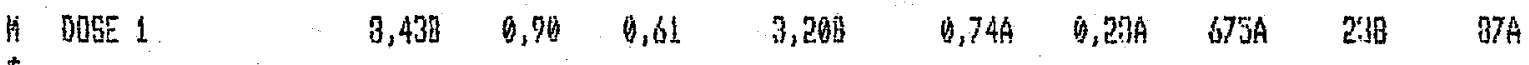

E.

D

I

A

5 DISE 2

$12,15 \mathrm{~A} \quad 0,98 \quad 0,56 \quad 3,56 \mathrm{~A} \quad 0,66 \mathrm{~B}^{\circ} \quad 0,2 / \mathrm{A} \quad$ 61.4A $\quad 172 \quad$ BOA
D.M.S.
$(1,17)$
$(6,14)$
$(0,47) \quad(0,62) \quad\{12 B)$
$\{23.57\}$

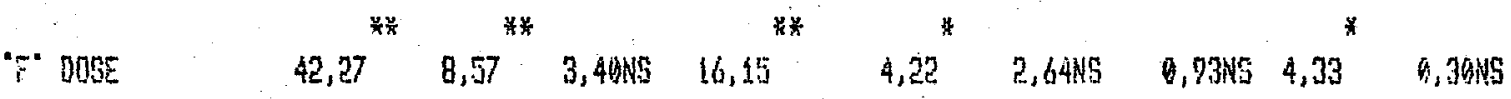

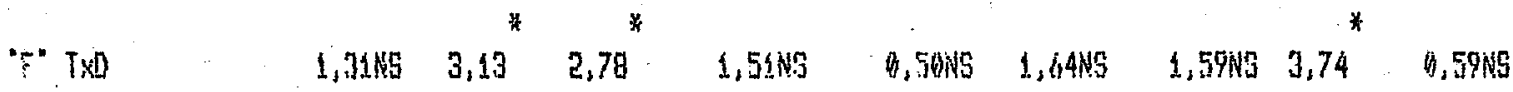


continuar:ău
ondro 7 -... Prometros
com interato
signitiontiva
entre
tratamento soses

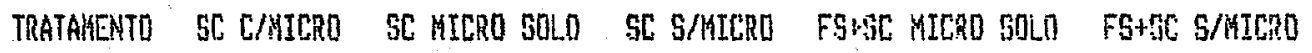
DDSE

NITROEENID \%

\begin{tabular}{llllll} 
DOSE 1 & $0,81 \mathrm{Aa}$ & $0,96 \mathrm{Aa}$ & $0,91 \mathrm{Aa}$ & $0,39 \mathrm{Ab}$ & $0,91 \mathrm{AB}$ \\
DOSE 2 & $0,92 \mathrm{Bz}$ & $0,89 \mathrm{a}$ & $0,921 \mathrm{a}$ & $1,96 \mathrm{ABZ}$ & $1,12 \mathrm{Aa}$ \\
\hline
\end{tabular}

0.4.5. (6.19) mailsculo 5\%

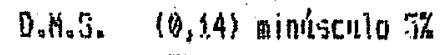
TUKEY

FUSEO0\%

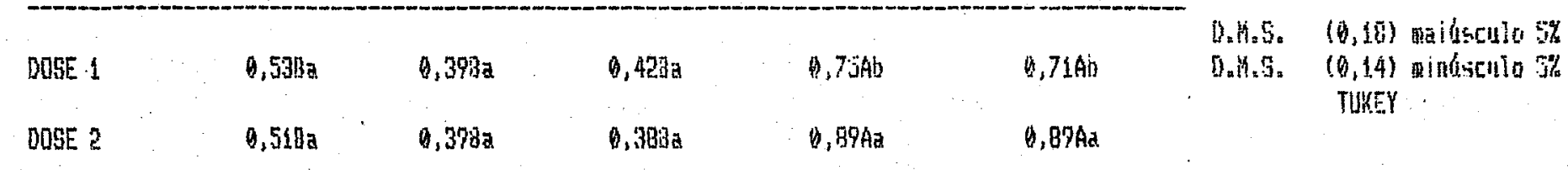

FER 0 ppq

\begin{tabular}{|c|c|c|c|c|c|c|c|}
\hline DOSE 1 & $1797 \mathrm{a}$ & $174 \mathrm{Ba}$ & $1428 \mathrm{z}$ & $1689 \mathrm{a}$ & $\mathrm{I} 13 \mathrm{Ad}$ & $\begin{array}{l}\text { D. H.5. } \\
0.4 .5 .\end{array}$ & $\begin{array}{c}(205\rangle) \\
(\{45) \\
\text { TUKEY }\end{array}$ \\
\hline DOSE 2 & 187 Az & 1525a & $141 \mathrm{Az}$ & $17 S A$ & $244 \mathrm{fh}$ & & \\
\hline
\end{tabular}




\subsection{Ensaio de $11 \times 1$ viaço}

Os remultados de analise de terra do ensaio de lixivi aco esota mepresentados no Quadro 8 (pag. 102) e Duadro 9 (pag. 103 0104 ).

Inicimlmente apresenteremos aquel as que

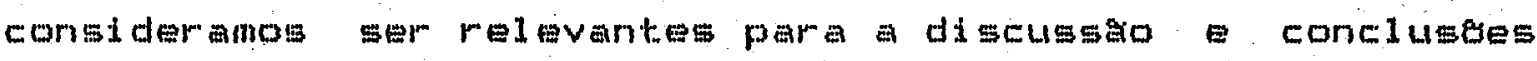
apresentadas na sequencia.

\section{3.1. Apresentma do dom remultados}

"Fosfora soluvel (ug/em 3 - F- Na profindidade um dos teores maj olevados de fosforo sol avel ocorrem nos tratamentos $\mathrm{SC} \mathrm{sem}(256)$ FS (286) entretanto nac diferem estatioticamente do tratamento sc com (163) que apresenta un valor de p mais baiso. A testemunha e inferior a todos os outros tratamenkos (s). Na profundidade dois

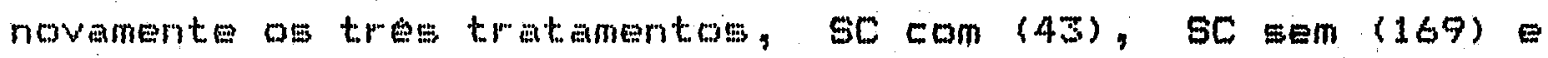

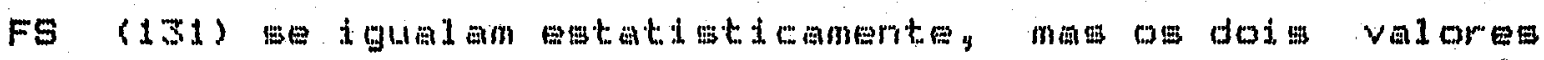

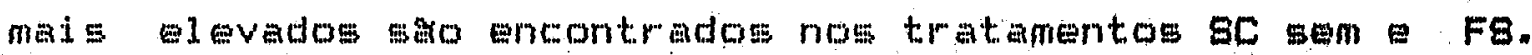

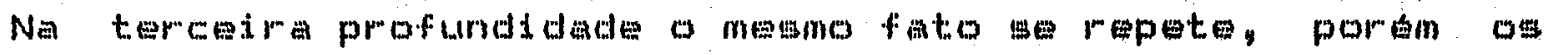
tratamentos SC com sC sm diferem estatisticamente sendo 0 valor mais bajso novamente associado ao tratamento SC com. A partir da profundidade quatro, ate a litima profundidade (sete), todos os tratamentos se igualan entre si a a 
testemunha, no sondo majs constatado o efeito dos tratamentom.

\section{3}

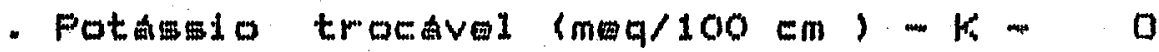
teor de potassio trocavel ate a profundidade tres e mais elevado e igual entre 51 nos tratamentos $5 \mathrm{cem} 10,99$ em media) EFS(0,92 en infolia), do que os kratamentos SC com

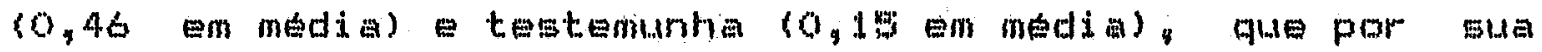

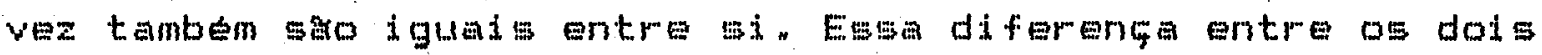
grupos de tratamentos forfirmada pela arise estatistica em quase todas as interaçesentre a es. A partir da profundidade quatro todos os tratamento se igualam entre si, e testemuntia inclusive.

\section{3 \\ "Cale10 trotavel (meq/100 $\mathrm{Em}$ ) - Ca -}

teor de calcio trocavel \& batante homogeneo se compararmos os tratamentos em profundidaden Apenas na profundidade Lim foi observado un teor sgnificativamente mais elevado no

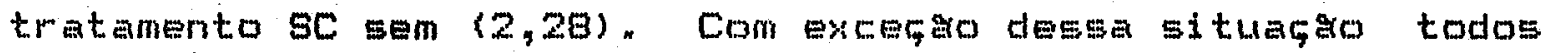

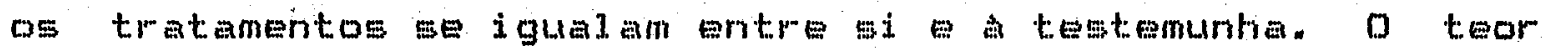

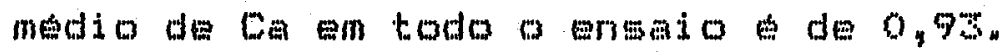

3

- Magnesio trocavel (meq/100 cm) - Mg - Ate

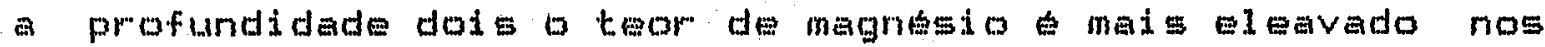
tratamentos SC sem 10,42 em media) e FS $(0,41$ em media), se comparados ac tratimento sc com (0,20 em media). EsEa 
diferenca estatisticamente significativa. A testemunha se iquala a tratamento SC com em todis as profundidades" A partir da profundidade tres todos os tratamentos $E$ a testemunha se iqual am entresi.

\section{3}

- Aluminio trocável (meq/em) - Al - Naro há interaço significativa entre tratamentos profundidade em relaço ab teor de aluminio trocavel em profundidade. Nato ha diferenca significativa do teor de Al ate a profundidade seis, apesar da beixa Diferenca Minina Significativa PDMS = s $(0,04) \mathrm{meq} 100 \mathrm{~cm}$ ). Em relaço aos tratamentos tambem houve pouca variaço. a teor mais elevado foi verificado no tratamento SC sem $(0,43)$. OE tratamentos SC Com $(0,39)$, FS $(0,42)$ e TEST $(0,37)$ ge igual am entre si. A Diferenga Minima Significative tamberm muito baise (DMS $=\{0,05\rangle \mathrm{meg} / 100$ $\mathrm{cm})$.

3

- Soma de bamelmeq/100 cm $)-s$ - Ate a profundidade dois a Soma de Eases da testemunha 1,34 em media) edo tratamento SC com $(1,73$ ein medi a) se iqual aram. o valor $S$ dos tratamentos SC sem fe fS for am, ressas mesmas profundidades, superiores a testemunta. A partir da profundidade tres nao ha mais efeito dos tratamentos em relaço a Soma de Bases, uma vez que todos os tratamentos se iqualaram a testemurita a ei proprios. 
A Saturacto por Eames a CTC tiveram

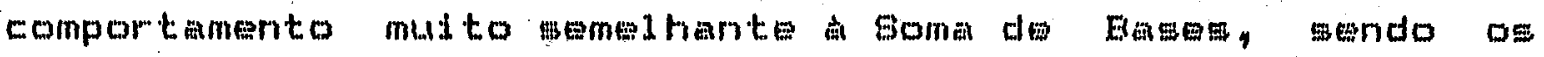
mesnos comentarios validos tamben para esse caso.

\section{3.2. D1}

D teor mais El evado de potassio trocavel nos tratamentos SC men $F E$ a semelhanga entre a testemuntia o tratamento SC com levamos a inferir que a aplicasăo de $k$ via SC faz on que Esse nutriente seja menos lisiviado se comparado a FS, ficiando consequetemente retido dentro do 5C. Apesar da grande quantidade de gesso (CaSO.2H D) adicionato as colunas ne forma de $5 \mathrm{C}(15,0 \mathrm{~g}$ por coluna) na 4

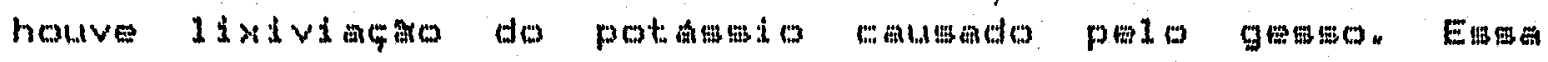
inferturia baseiame no fato do teor de potassio ser semelhante nos tratamentiog FS SC mem, mondo que ambos tiveram adiço de 46 ppm de k-moH por coluna no solo. mplicado da mesma forma e, apenas o tratamento $S C$ sem recebeu adiça de 15,0 g de 9 esso na forma de $8 C$ men $N, P$ e K.. Caso gefe getso estivese Iixiviando o potaseio os teores de potassio do tratamento GC wo possivelmente tambem do SC com, deveriam ser mais elevados em profundidade. ENGELSTAD \& RUSSEL (1975) alertam para o fato da lisiviaca ser menos intensa para as formas FSL de potassio quando comparadas aos fertilizantes potassicos a tiamente mol Liveis. 
o comportamento do mo, toores ma is elevados nos tratamentos SC sen a FS ma is ba ixos no tratamento so com testemunha, tamben nos levam a presumir aue näosteja havendo lixiving do manesio devido do preseno do 98500

oteor de chicio, bastante un forme entre os

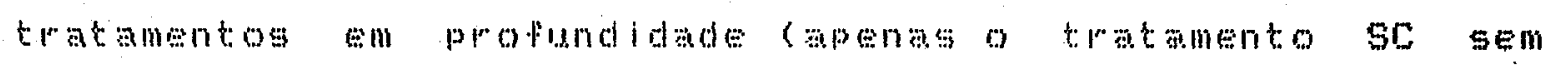

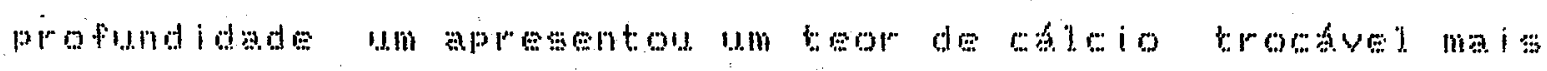
elevado, os outros tratementos nessa protundidade e todos os tratamentos, a teatemuha inchus ive, nas outras mostramam

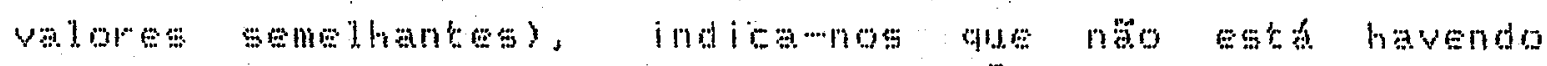

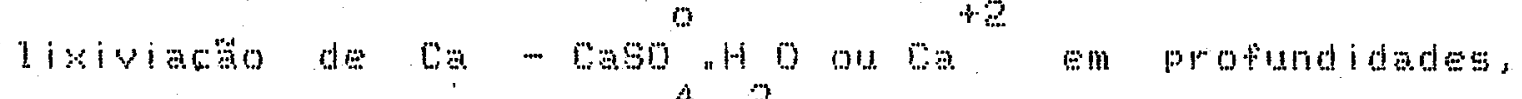

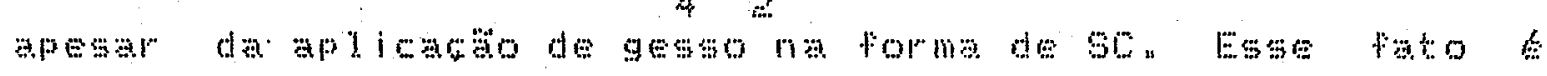
confirmado pelo comportamento do $A l$, isto b, no ha diminuliso do toor de Al trocbvel nos tratamentos com

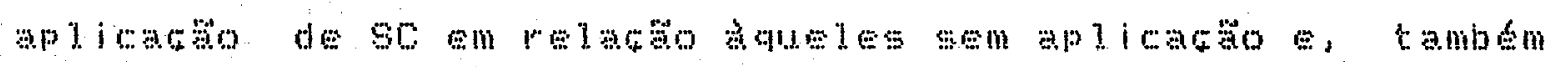

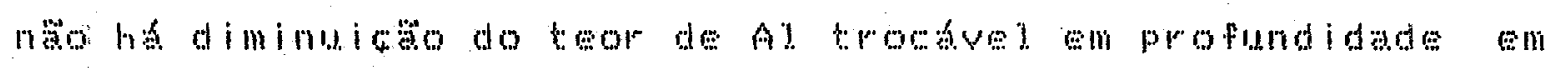

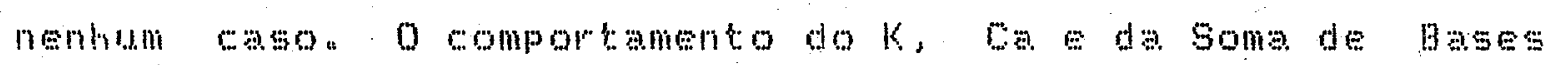

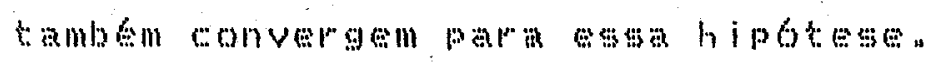

A Difereno Minima signipicativa bastonts Elevada no aso do postoro cops entre tratamentos (132) ugp/cm) torna dificid o apareimento de diferengas significativas centre os tratamentos Basendomos no

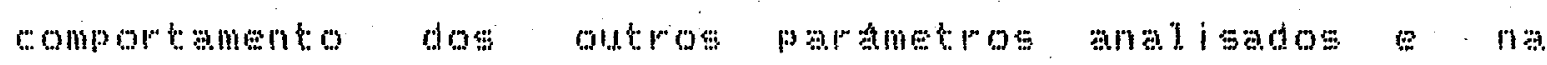


tendencia do valor nominal dos teores de fosforo trockvel tecorsmos a gun coments

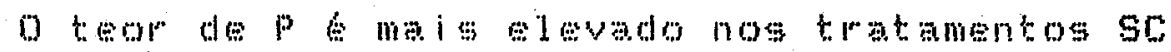
$5 \mathrm{em}$ \& FS em relaro aos tramontos SC con profuntide tresto lna profundiade tresoteor de p gignificativamente superior no tratamonto sc sem, em relaga

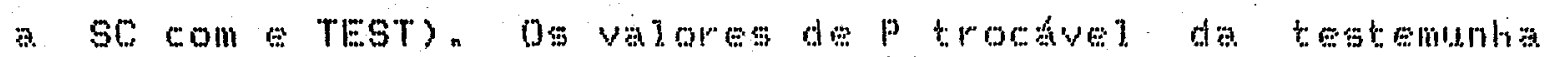
tornam-se muito proximos dos do tratamento so com a partir

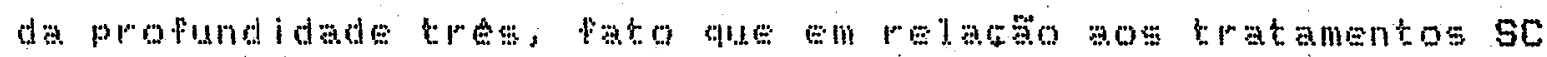

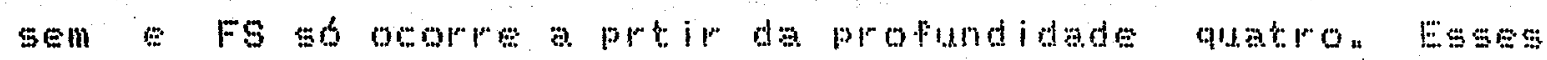

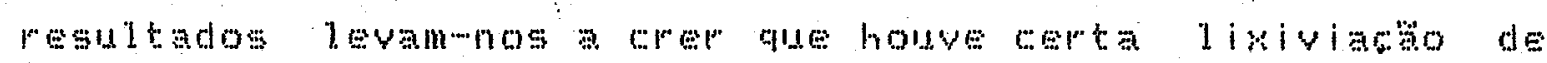
Hotoro nos fratamentos SC sem o FS, mrovavelmente por Plaro

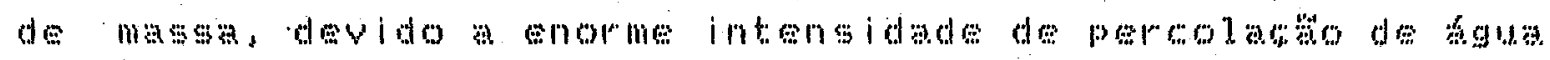

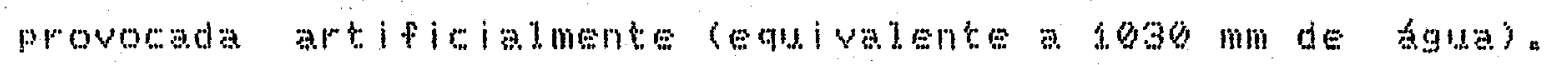

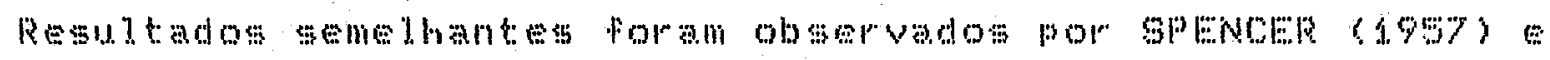
HUMPHIERYS a PRITCETT (AP7T)" No tratamento SC com, provavelmente osa lixiviago bor theo de mass foi menos intensa cos valores de p sabo mas baxos a diminuem

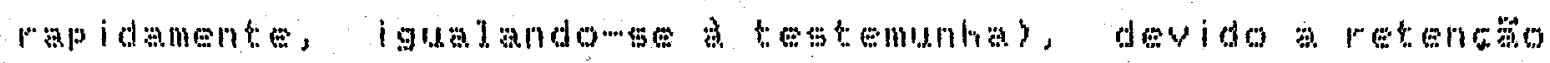

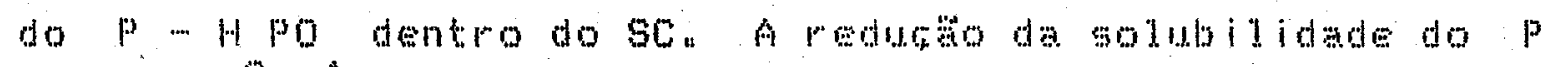

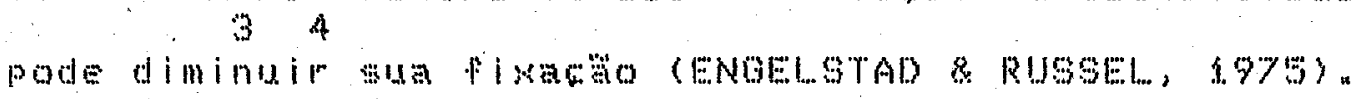




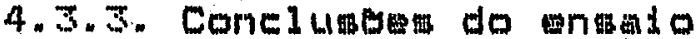

a) aplicacto de gesso na forma de SC nas condiçesesperimentais, nâmo provorou a lisiviag aro de outros cations $\left\{k^{+}, \mathrm{Mg}^{+2}\right.$, nem a neutralizaca do $A 1^{+3} \mathrm{em}$ profundidade:

b) O F́ aplicado via 50 provavel mente tamben o. F, ficam retidos parci almente dentro do sc, interagindo menos com o meio sendo consequentemente lixiviados e liber-ados em menor intensidade

(i) a metodologia adoteda permitiu testam a

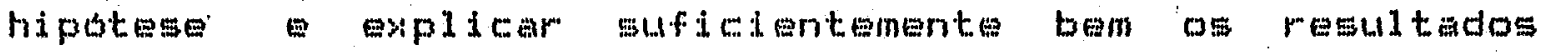
obtidos. 
ouaro 8 - Resultados de Andise de Terra do Enaro de Lixiviata - Médias e Testo

\begin{tabular}{|c|c|c|c|c|c|c|c|c|}
\hline pH $\quad M$ & $p^{8}$ & $y$ & Ca & $14 \mathrm{~g}$ & $\mathrm{Al}$ & $H+A]$ & $\mathfrak{s}$ & $T$ \\
\hline
\end{tabular}

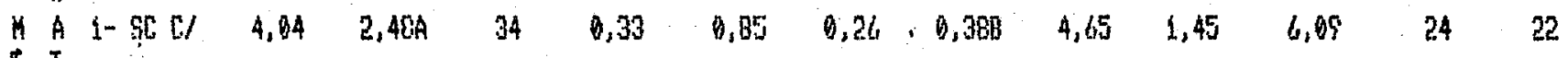

\begin{tabular}{lllllllllllll}
\hline & $T$ \\
A $2-5 C 5 /$ & 4,28 & $2,52 A$ & 85 & 0,59 & 0,57 & 0,33 & $6,43 A$ & 4,22 & 1,69 & 6,12 & 29 & 22
\end{tabular}

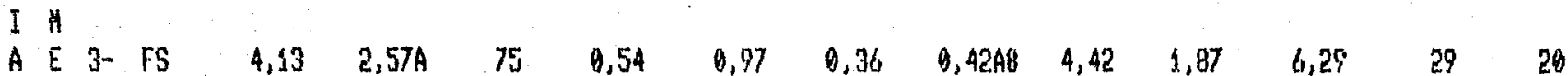

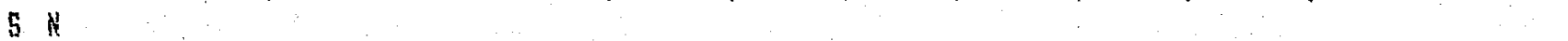
$\begin{array}{llllllllllllll}T & 4-T E S T & 4,18 & 2,54 A & 5 & 0,16 & 0,94 & 0,33 & 9,378 & 4,16 & 1,41 & 5,57 & 25 & 21\end{array}$ 5

0.14.5. $\quad(0,48)$

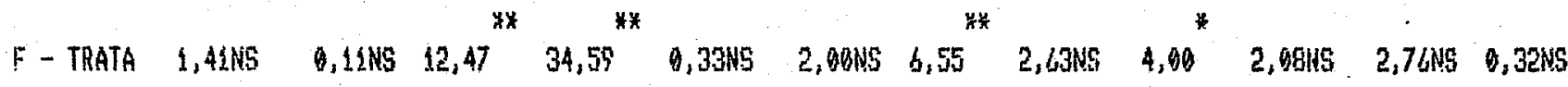

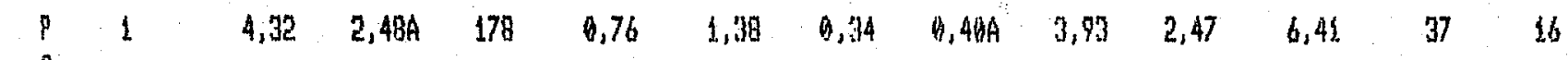

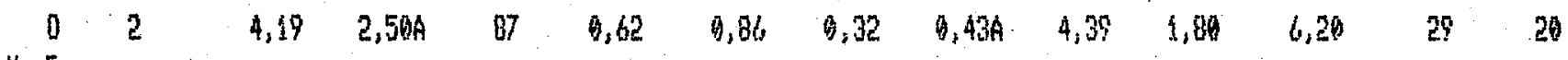

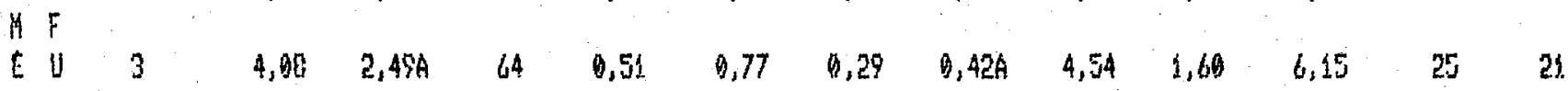

DN N

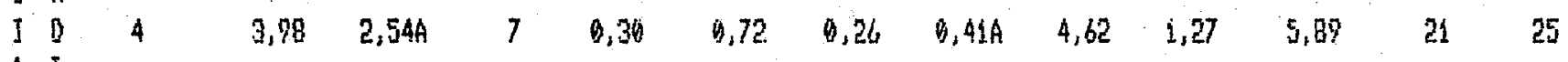

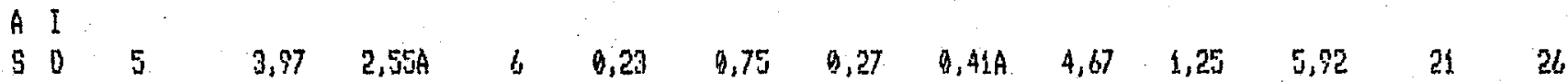

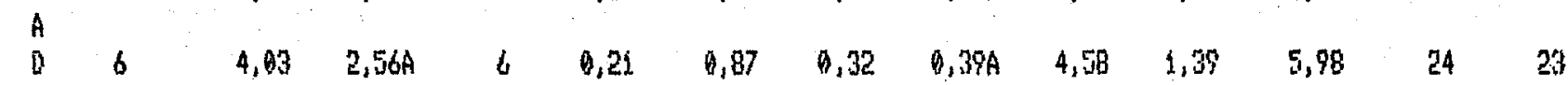

E

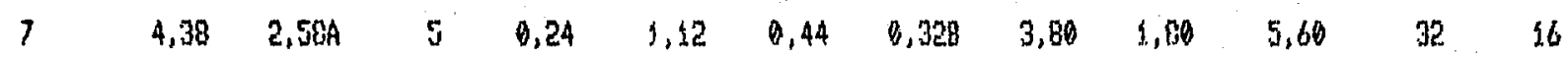

D.H.5. $\quad(0,14) \quad(9,94)$

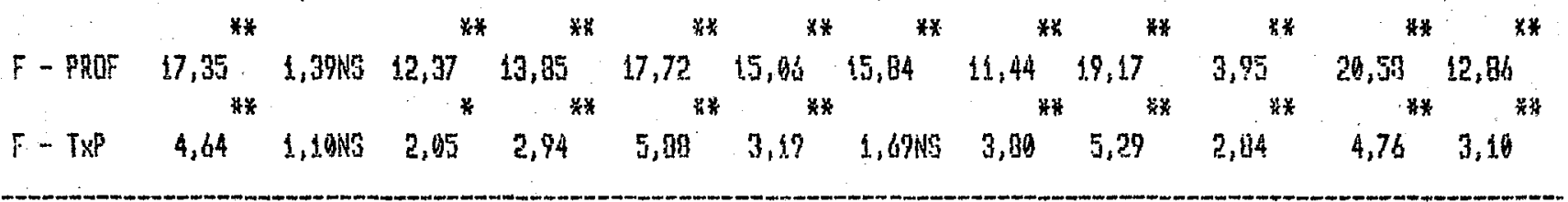

* TUMEY $5 \%$

** TUKEY $1 \%$ 
euadro 9 - Resultados da Analise de Terra do Ensalo de Lixiviacăo -.- Resultados com interamo simitiotiva oritre rratamentos profund idade

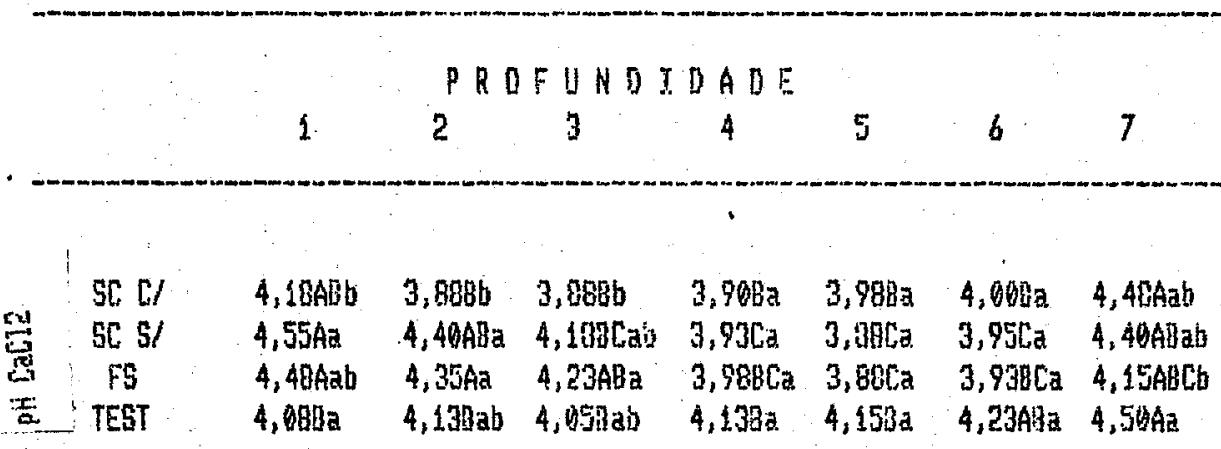

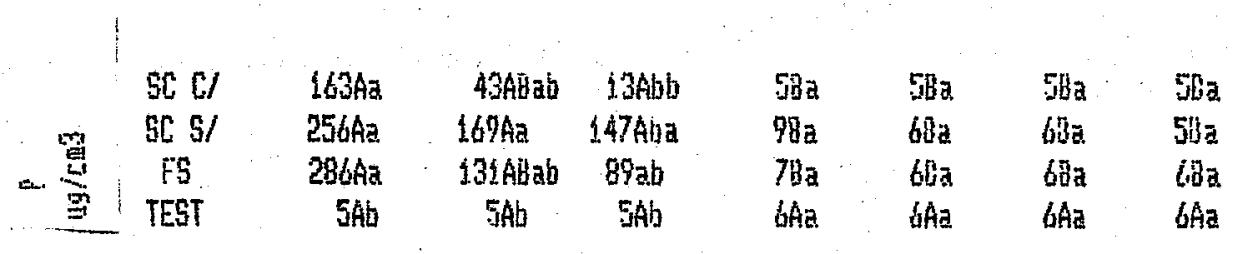

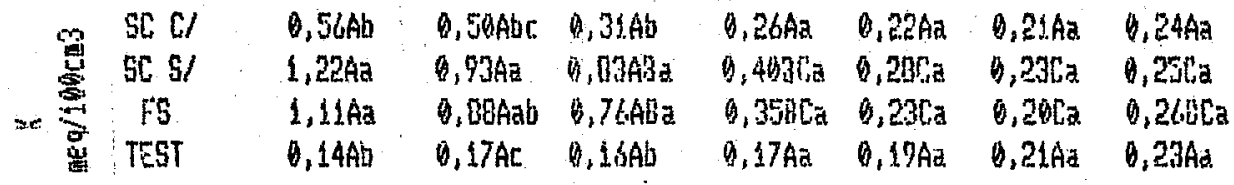

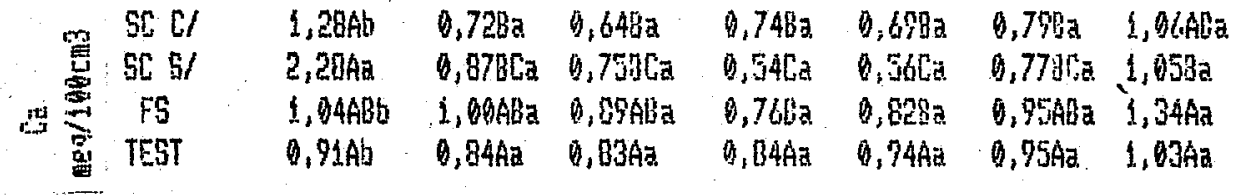

\begin{tabular}{|c|c|c|c|c|c|c|c|c|}
\hline & $5 C[1$ & 0,1886 & $0,228 \mathrm{~b}$ & $4,22 \mathrm{Ba}$ & $0,278 \mathrm{a}$ & $0,26 \bar{B}$ & $0,29 \mathrm{ABZ}$ & $0,49 \mathrm{Aa}$ \\
\hline & SC \$ $/$ & $0,46 \mathrm{Aa}$ & ๑, 3BABة & $0,32 \mathrm{BCa}$ & $0,24 \mathrm{Ca}$ & $4,21 \mathrm{CZ}$ & $0,39 \mathrm{Bl}^{\mathrm{a}} \mathrm{d}$ & $0,43 \mathrm{Aa}$ \\
\hline & TEST & $\begin{array}{l}n, 41 \text { As } \\
6,32 \mathrm{~A} a \mathrm{ab}\end{array}$ & 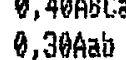 & $\begin{array}{l}9,33 \mathrm{BCD} \\
0,27 \mathrm{Aa}\end{array}$ & $\begin{array}{l}1,260 \mathrm{a} \\
0.27 \mathrm{Aa}\end{array}$ & $\begin{array}{l}0,27 \mathrm{CDa} \\
0.37 \mathrm{Aa}\end{array}$ & 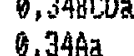 & $\begin{array}{l}0,50 \mathrm{Aa} \\
0.49 \mathrm{Aa}\end{array}$ \\
\hline
\end{tabular}

- As letras mailisculas indicam diferenca signnificativa na hor izontal (TUKEY 5y)

- As letras aindsculas indican diferenca significativa na vertical (TUKEY 5y) 
cantinuacลิ

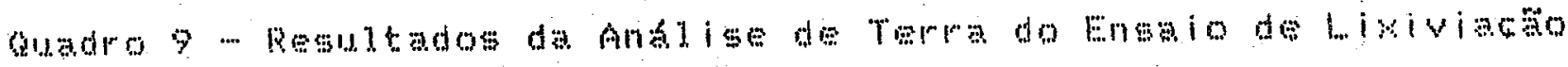

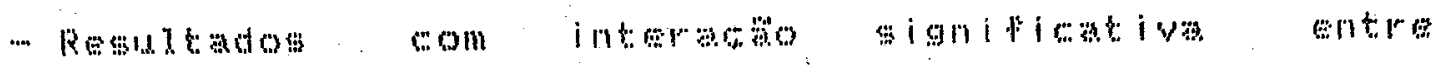
Tratamentose Protundidade

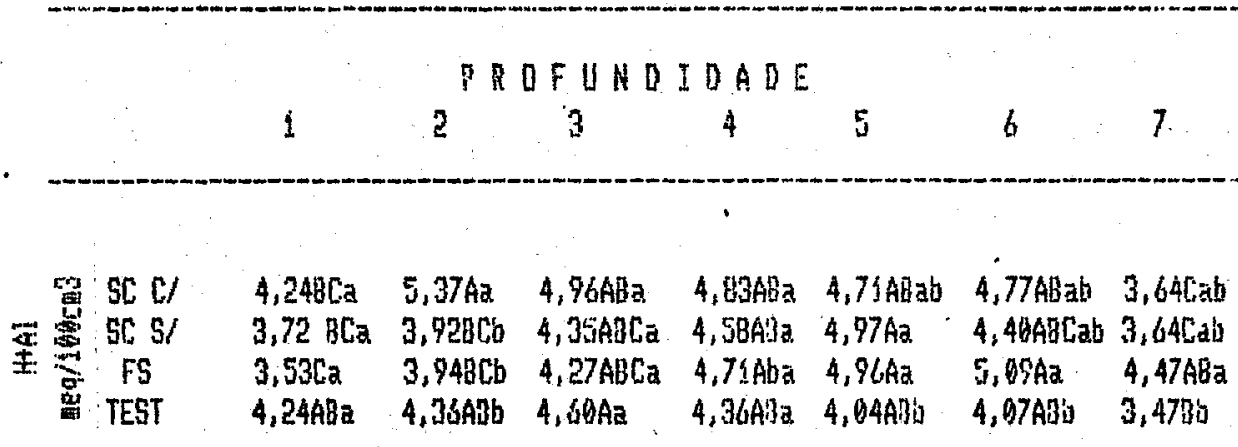

\begin{tabular}{|c|c|c|c|c|c|c|c|c|}
\hline & SC. C/ & 2, QBAADE & $1,44 A B b D$ & $1,27 \mathrm{ABZ}$ & $1,26 \mathrm{Aba}$ & $1.17 \mathrm{a}$ & 4.28ABa & $1,70 \mathrm{AGa}$ \\
\hline & 565 & $3.95 \mathrm{Aa}$ & $2,198 \mathrm{ah}$ & $1,88 \mathrm{BCz}$ & $1,150 \mathrm{E}$ & $1,05 \mathrm{Ca}$ & $1,39 \mathrm{Ca}$ & $1,758 \mathrm{C}$ \\
\hline 蹗 & FS & $2,56 \mathrm{Ab}$ & $2,2 \mathrm{EABA}$ & $1,79 \mathrm{ABC}$ & $1,36 \mathrm{~s}$ & 1. $31 \mathrm{Ca}$ & $1,49 \mathrm{PCa}$ & $2,10 \mathrm{ACO}$ \\
\hline & TEST & $1,37 \mathrm{AC}$ & $1,31 \mathrm{AC}$ & $1,2 \mathrm{BAZ}$ & $1,99 \mathrm{Az}$ & $1,46 A 2$ & $1,59 \mathrm{Az}$ & 1, GUAA \\
\hline
\end{tabular}

\begin{tabular}{|c|c|c|c|c|c|c|c|}
\hline 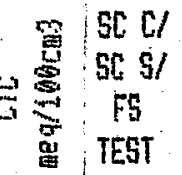 & $\begin{array}{l}6,26 \mathrm{ABb} \\
7,67 \mathrm{Aa} \\
6,69 \mathrm{Ab} \\
5,61 \mathrm{Ab}\end{array}$ & $\begin{array}{l}6,81 A a \\
6,978 \mathrm{a} \\
6,22 \mathrm{Aa} \\
5,67 \mathrm{Aa}\end{array}$ & $\begin{array}{l}6,23 \mathrm{ABa} \\
6,239 \mathrm{a} \\
6,25 \mathrm{Az} \\
5,38 \mathrm{Aa}\end{array}$ & $\begin{array}{l}6,09 A B a \\
5,748 \mathrm{a} \\
6,07 \mathrm{Aa} \\
5,66 \mathrm{~A}\end{array}$ & $\begin{array}{l}5,96 \mathrm{Bba} \\
6,62 \mathrm{az} \\
6,27 \mathrm{Aa} \\
5,51 \mathrm{Aa}\end{array}$ & $\begin{array}{l}6,66 \mathrm{ABa} \\
5,67 \mathrm{Aa} \\
6,58 \mathrm{Aa} \\
5,57 \mathrm{Aa}\end{array}$ & $\begin{array}{l}5,33 \mathrm{~b} \\
5,398 \text { ab } \\
6,57 \mathrm{~A} a \\
5,13 A^{\prime}\end{array}$ \\
\hline
\end{tabular}

\begin{tabular}{|c|c|c|c|c|c|c|c|c|}
\hline 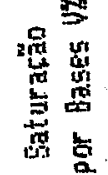 & $\begin{array}{c}\text { SC CI } \\
\text { SC S/ } \\
\text { FS } \\
\text { TEST }\end{array}$ & $\begin{array}{l}31 \mathrm{ABbC} \\
51 \mathrm{AB} \\
42 \mathrm{Aab} \\
25 A C\end{array}$ & $\begin{array}{l}21 A B b \\
36 A a \\
37 A a \\
23 A b\end{array}$ & $\begin{array}{l}296 \mathrm{~b} \\
269 \mathrm{COAb} \\
32 \mathrm{AB} \\
22 \mathrm{~A}=\mathrm{b}\end{array}$ & $\begin{array}{l}\text { 21Aba } \\
200 a \\
238 a \\
23 A a\end{array}$ & $\begin{array}{l}29 \mathrm{Ba} \\
290 \mathrm{a} \\
21 \mathrm{Ba} \\
27 \mathrm{Aa}\end{array}$ & $\begin{array}{l}22 \mathrm{ABa} \\
23 \mathrm{CO} \mathrm{a} \\
23 \mathrm{Ba} \\
27 \mathrm{Aa}\end{array}$ & $\begin{array}{l}32 \mathrm{Aa} \\
329 \mathrm{AC} \\
32 \mathrm{AB} \text { a } \\
32 \mathrm{~A} A\end{array}$ \\
\hline
\end{tabular}

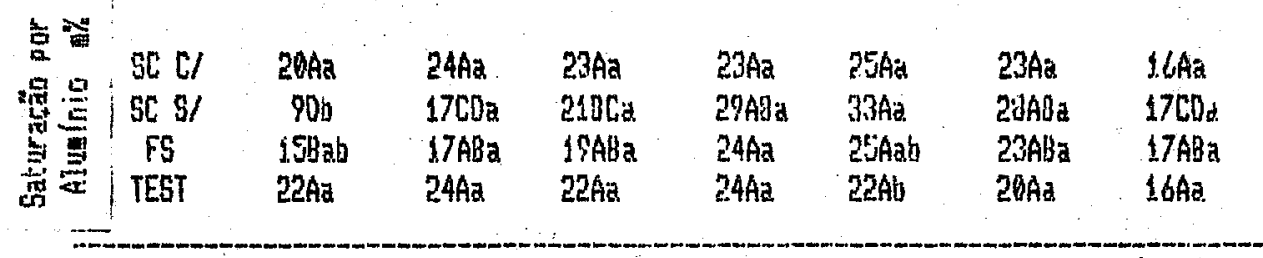

- As letras maiúsculas indican di ferenca significativa na hor izonta! (TUKEy 5\%) - As letras ainúsculas indican diferenca significativa na yertical (TUKEY 5\%) 


\section{CONCLUSGES}

Como conclusbes gerain dos tres ensaios podemos citar:

a) D SC naัo se comportou como um Fertilizante de Solubilizaço Lenta, porem nao contribuil negativamente na producaso das plantes:

b) a eplicaço de mitronutrientes via $S C$ e eficiente e víavel ;

c) o efeito do modo de aplicaga dos micronutrientes restringewse um cultivo. A aplicacaso de micronutrientes via sC apresenta vantagens em relacăo a aplicaça no solo apenas no primeiro cultivol

d) a aplicacoso de gesso na forme de SC nao provocou a lixiviaço de $k$ ou $M g$ do solo nem a

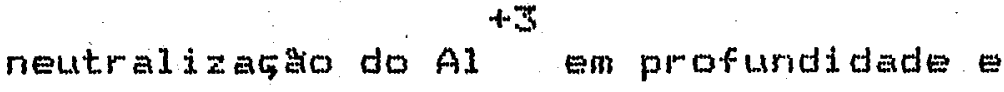


e) D SC provoca aumentos do teor de $\mathrm{P}, \mathrm{Ca}$ a

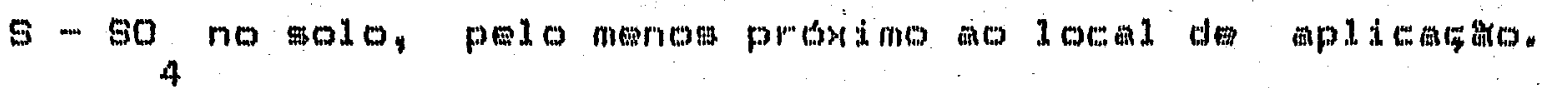




\section{REFERENCIAS BIBLIOGRAFICAS}

ALLISON, F.E. The fate of nitrogen applied to boilm. Advanceg in Agronomy, 18: 219-258, 1966.

ATANASIU, N. Zur Wirkung schwerloslicher $N$ - Verbindungen. 7. Pfanzenern. Dung. Eodentunde, 84 (1-3): 103-110, 1959.

BACHE, G.W. \& HEATHCOTE, R.G. Long-term effects of fertilizers and manure on soil and leaves of cotton in Nigeria. Experimental Agriculture, 5: 241-247, 1969.

EATAGLIA, O.C.; FURLANI, A.M.C. TEIXEIRA, J.P.F.; FURLANI, F.F. \& EALLO, J.A. Metodoe de analise quimica de plantas. Eol. Téc. no. 78, Instituto Agronomico. Campinas-SF, 48p. 1983.

EEATON, J.D. HUEEARD, W.A. \& SPEER, F.C. Coated Hrea, thiourea, umearomaldehyde, hexamine, oxamine, glycoluril, and oxidized nitrogen-enriched coal as slowly available sources of nitrogen for orchardgrass. Agron.,59(2): 127-133, 1967. 


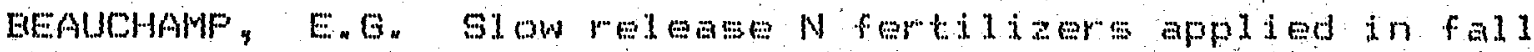
for comr. Can. J. Soil Sci., 57, 487-496, 197\%.

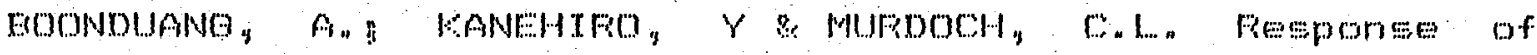
"sunturf" bermudapese to slownelease nitrogen sources under grentouse conditione. Hort Seience, $11(4): 379-$ 381,1976

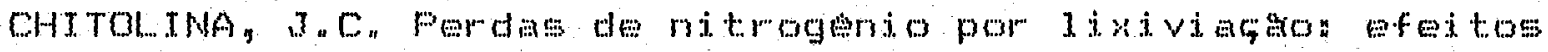

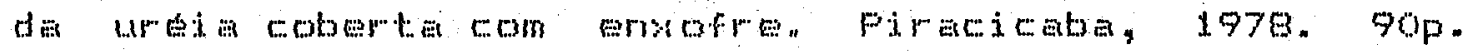

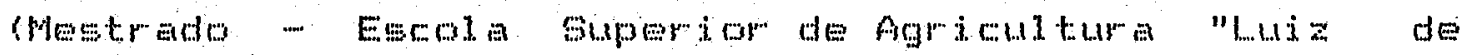
Mus i raz" Ausp?

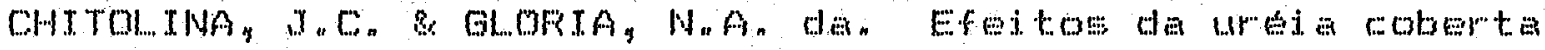

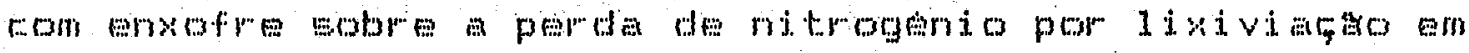
um Latossol Bono" Anats da E.B.A. "Ludz de Quejroz",

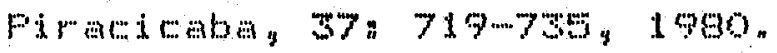

DALAL y R.F. Comparative efficiency of soluble and

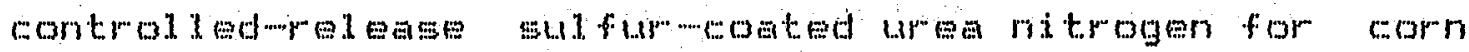
in tropics. Soil Sci. Soc. Am. Proc. 38:970-974, 1974. 
DALAL, F.C. DATTOO, R.F, \& INDALEINGH, T. FElative

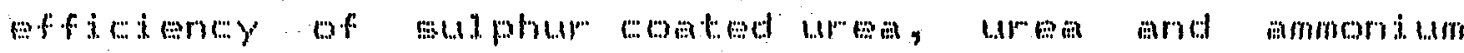
sulphate in a calcareous goil. Tropleal Agritulture,

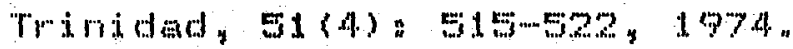

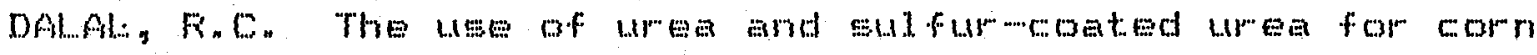
production in a tropical soil. Soil Sci. Sot. Amer. Frot , 39: 1004-1005, 1975.

ENUEISTAD, O.F. \& FUSEEL, D.A. FertilizerE for LSE Under tropical ronditions. Advariese in Agmonomy, 27: 175-200, $197 \%$

FAD. Fertilizer and plant nutrition guide "Euldetin 9. In: - Fome, FAO, 1994, P. Bs

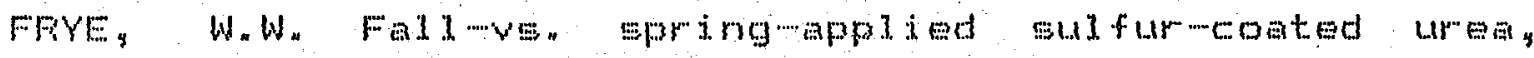

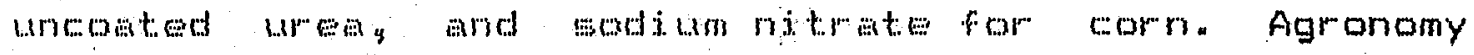
Journa1, $69: 278-292,1977$

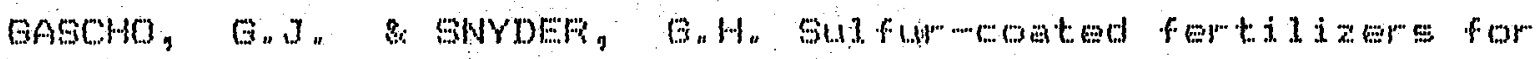

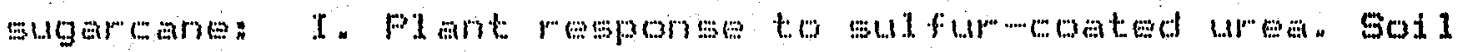
Sci. Soc. Am. 3., 40, 119-122, 1976. 


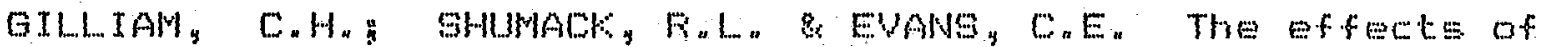

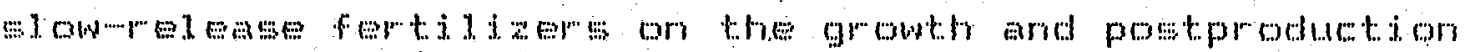
performance of Eoston ferr. Hort seience, 18(4): 442-444, 1993

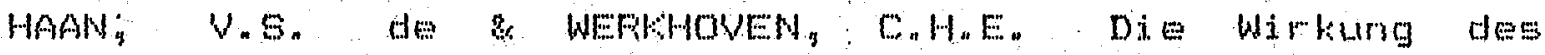
Stickstoffs in langsammirkender Eynthetisch-organischen Stictotoffuengemiteln oder Mischomengern mit einer

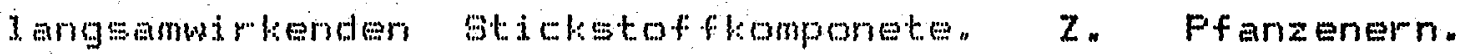

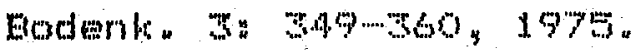

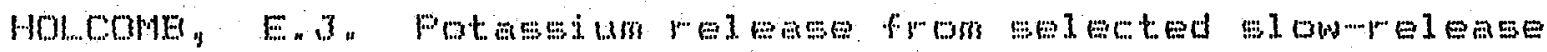

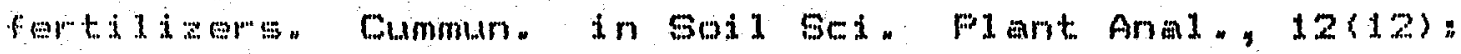
$1305310,1931$.

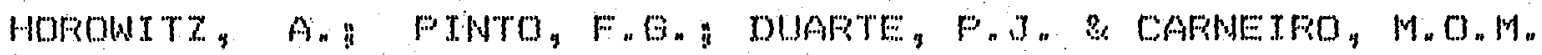
Froduca de adubos de solubilidade controlada. Rev. bras. Ci. Solo, 2:144-147, 1978.

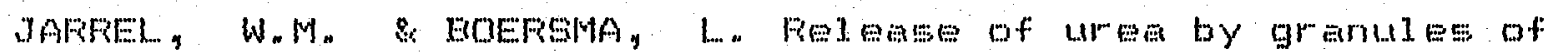

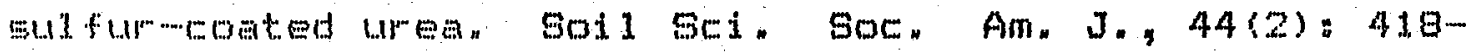
$422, \quad 1980$.

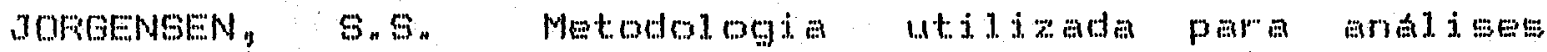
quimicas de motina. Gui a analtico. Piracicaba, Centro de

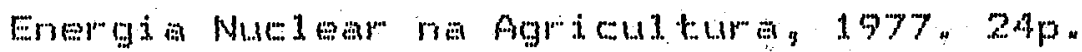




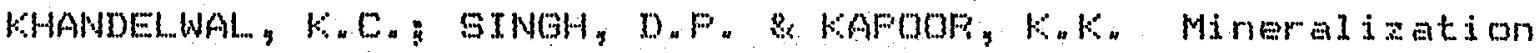

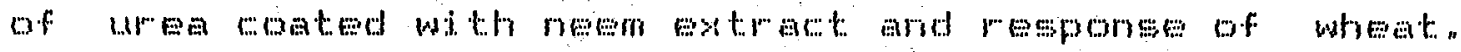
Indian 3. mgric. Sei. 47(6), $267-270,1977$.

LIEDEL, E.A. \& WALSH, L. M, Evaluation of Euf fur conted ur a (9ou) applied to irrigated potatosen and com. Agronomy Journal, $68: 457+43,4976$.

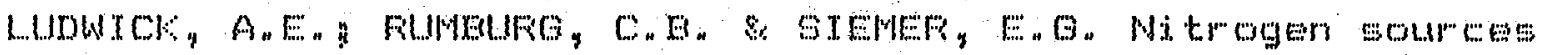
for hay production on flooded meadows "Soil Sei. Sor. Am. 3., 42:509-512, 1979.

LUNT, O.F. \& OEFTLI, 3, I. Controlled releate of fertilizer miner al s by incapsulating membranes. II. Efficiency of recovery, jnfluence of soil moisture, mode of application, and other consterations related to use. Soil Sci. Soc. Am. Frot., 26. 584-587, 1962.

MAOUIETRA, A." OLIMENT, M.D. "FUCHADES, R. \& FRIMO YUFEFA,

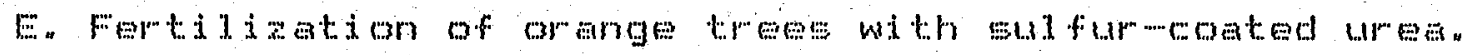
Nitrogen levels in lewese and frutes plant and soil, $90(2): 247-254,1964$. 
MArochA, JE" Ammonia wiatilization and nitrogen

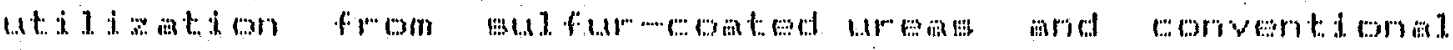

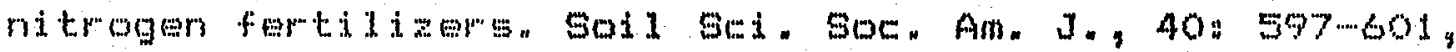
1976

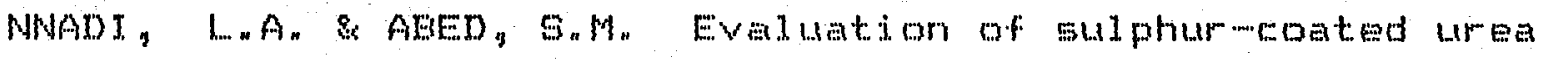
fertilizers in savannah soils 1. Response of totton and Sorghum. J. agric. Sc1. y 101" 1.9-123, 1983.

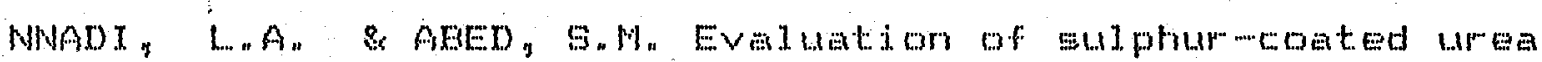
fertilizers in savanmath soils 2. Effects on soil pH and

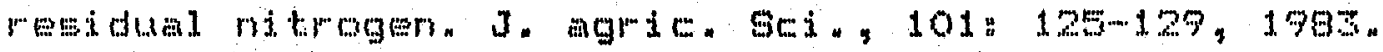

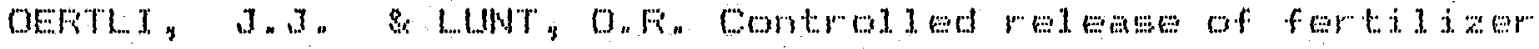
minerals by incapsulating membranes" I. Factors influencing the rate of relemse"Soil Sci. Soc. Am. Froc, $26 \% 579-583,1962$

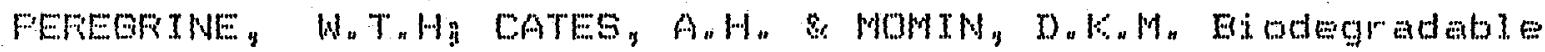
cellophane bags -... a controlled release gytem for pest and disease control and fertilizer application in

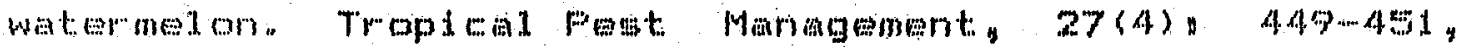
1981. 
FFASAD, F. FATALE, G. B. \& LAKHDTUE, E.A. Nitrification

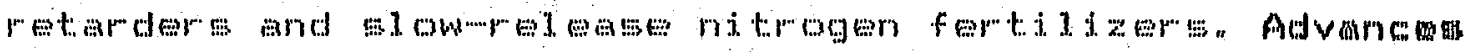
in Agronomy, 27: $357-393,1971$,

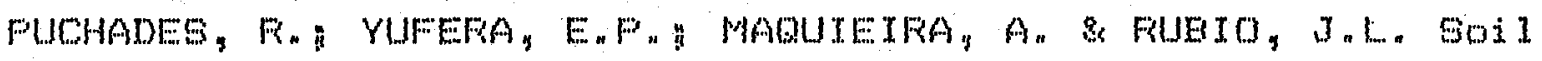
nitrogen flutuations in witmo plots fertilized with sulfur wated Limea and ammonicum nitw sulfate plant ard Soi1, 79: $357,365,1984 a$

PUCHADES, F." VUFERA ${ }_{4}$ E.F." \& RUETO, J.L. ThE release,

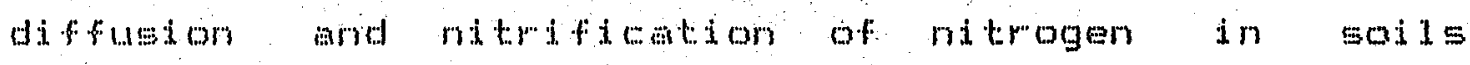
sur romding sulfurmoated wrea gramles. Plant and Soil, $75: 345-356,1984 \mathrm{~b}$

Rata, B. Yan o ouAgero, JA. Metodos de analise de eolo para fins de fortilidide. Fol. Tew. no. G1, Instituto

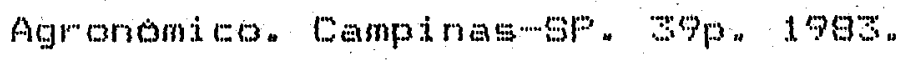

REDDY, R.N.S. \& FASAD, R. Studies on the mineralization of

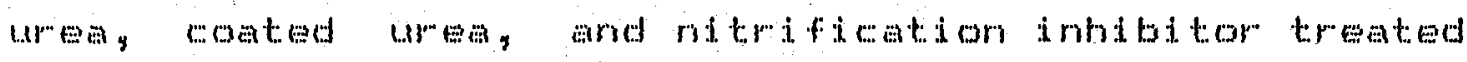
urea in soil. Joumnal of Soil Seience, 26(3)" 304-312, 1975 


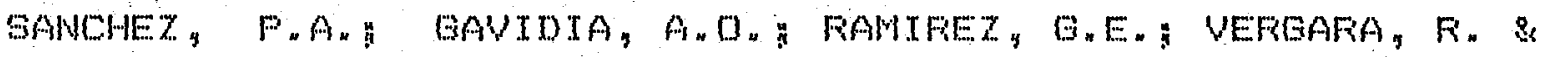

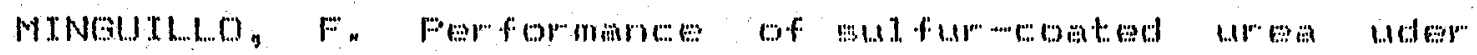
intermittontly flooded wice culture in Peru. Soil Sci. Soc. Aner. Proc. , 37: 769.792, 1973.

SANCHE7, F.A. Suelos del tropito. Carmeterigticas y manejo. Instituto Interameritiano de copperacion para 1 a Agriculturan San Jom, Costa Rica, 1981. p.55.

SARFLIEE, J.F. \& HAAG, H.F. Andises quimicas en plantas" Fipacicata - EsALQ, $1974,57 \%$, (mimeografado)

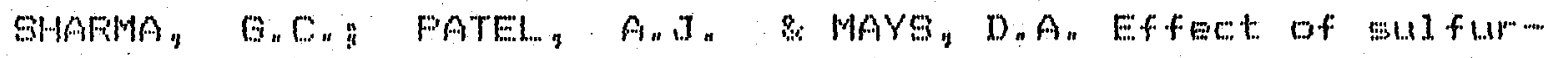
coated urea on yista, N uptabe, and nitrate content in turnip grems cobbage y and tomato J. Amer. Soc. Hort. $503,101(2), 142 \cdots 145,1973$

SHARMA, 5.M. WFASAD, R. EFFEt of rates of nitrogen and relative efficiency of subpurwoteded urea and

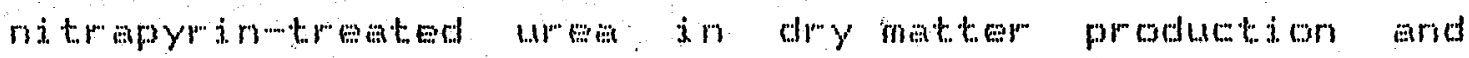
nitrogen uptake by rite. Flant and 9011, 55: 399-396, 1980.

SHELTON, 3.E. An Evaluaton of sublumconted umea as a preplant total season nitrogen supply for trellised tomatos. Soil Sei. Soc. Am. J., 40(1)" 126-129, 1976. 


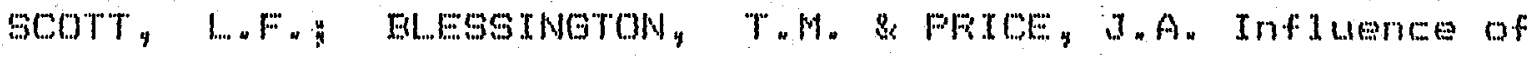

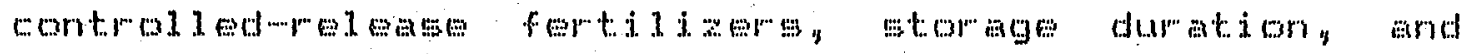
light source on posthamest quality of poinsettia. Hort Stience, 19(1): $111-112,1984$.

SNYDER, G.H \& YOUNG, T.W. Fertilizer poctets, fertilizer tablets, and a platste mul wh methow for flower production of fielu grown roses. I. Amer. Soc. Hort. Sci., 99(5)" $270-278,1974$.

SWVOER, G.H" a BACHO, G.I. Sulfur-tomted fertilizers for

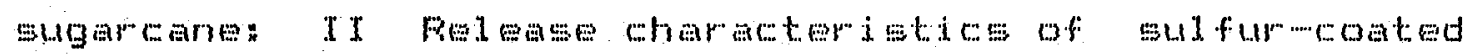
when and fCl. Soit Sci. Soc. Am, J., 40(1): $119 \ldots 122$, 1976

STONE, M"H, \& WTLD, An Fate of nituification of amomiated

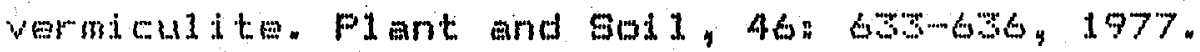

8

TEFMAN, G.L. ALLEN, G.E. \& ENOELGTAD, O.F. FESPOHEE bY paddy in ine to rates and soumes of apolded phosphomus. Agronomy Journa y 62: $390-394$, mai jown, 1970.

TEFMAN, 6.1." \& MORTVEOT, 3.3. Wutminnt Gffetiveness in $r$ lation to rates applisd for pot experimente: 1 . Nitrogen and potaswim. Soil Sci. Soc. Am. J., 42, 297302,1978 


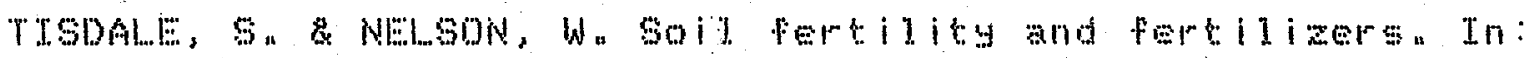

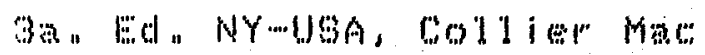

Millan International Editions, 1974 " p.378-379"

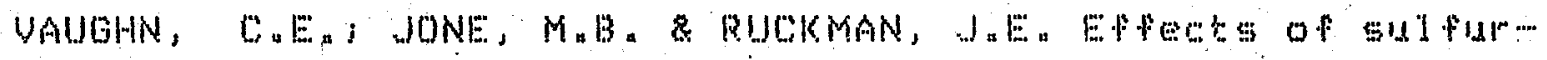
woted wrea on Calitornia annul grasuland yield and themical compositon. Agronomy Journa1, 71: 297-300, 1979.

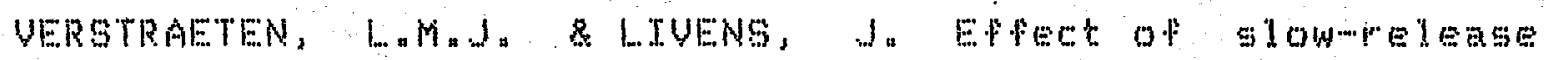

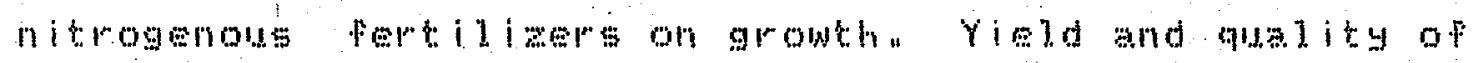
winter whest. $Z$. Pflanzemern. Bodenkunde, 4/5: 435m446, $1.77 \%$

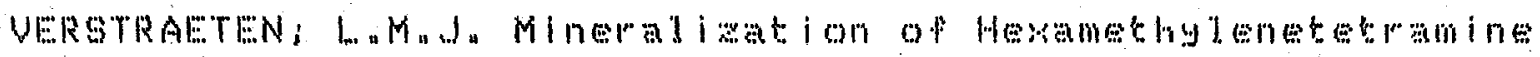
in soils.: Z.. Pfianzenern. Bodenkd., $140: 175 \ldots 181$. $1.97 \%$

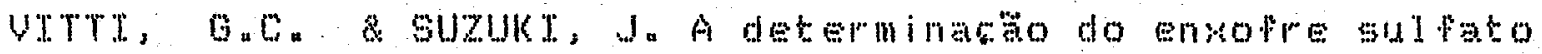
do solo pelo metodo tubidimetrico. Jabotimaba, FLA, 1978, 130, (mimeograpado)

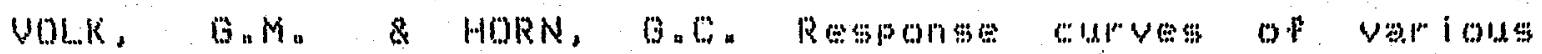

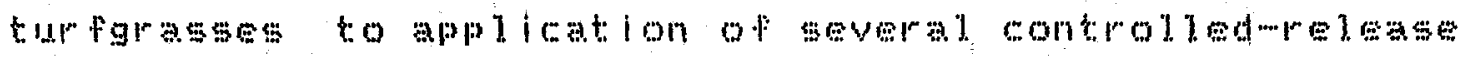
nitrogen sourom agronomy Journal, 67: 201.2004, 2975. 


\section{1}

WADDTNGTON, D.V" \& DUTCH, JM" EValuation of Sl ow

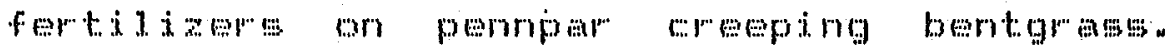

maromony Journa1, 69: 612-815, 5et-out. , 1976 .

WALKEY, A. \& ELACK, I.A. An Examination of the Degtjartf method for detemining soil organie matter; and proposed modification of the chromice ateid titration method soil SEience, $37: 29-38,1934$.

WATSCHKE, T.L. \& WADDINGTOW, DV. Effect of nitrogen EOUFE, raten and tining on growth and carbohydrates of Merion E1 uegras. "Agronomy Journmi, 66" 691-696, 1974"

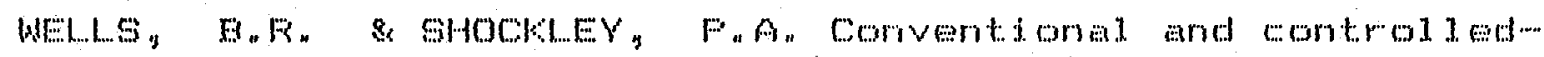

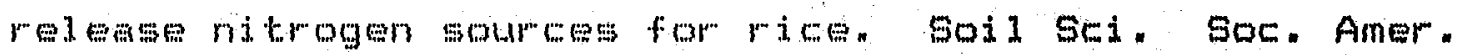
Proc, $59(3)$ : 549-5i, 1975 